\title{
Multidimensional (MD) Circuits and Systems for Emerging Applications Including Cognitive Radio, Radio Astronomy, Robot Vision and Imaging
}

Arjuna Madanayake, Chamith Wijenayake, Donald G. Dansereau, Thushara K. Gunaratne, Leonard T. Bruton, and Stefan B. Williams

\section{Ahstract}

Advances in the performance of VLSI circuits are leading to a number of emerging applications of multidimensional (MD) filters. Early progress was focused on the numerical design of two dimensional (2-D) transfer functions and the challenging stability issues associated with low-complexity infinite impulse response (IIR) implementations. However, over the last decade or so, important practical advances have occurred in the design of 3-D and 4-D IIR filters, leading to some important emerging applications. In this tutorial article, some of these applications are described, with emphasis on 2-D spatio-temporal beamforming and 4-D light field processing. In particular, advances in spatio-temporal beamforming for cognitive radio systems and for synthetic aperture radio telescopes are considered. In the 4-D case, we describe a class of 4-D light field filters for image processing, 4-D hyper-fan filters for low-light imaging, depth filtering, denoising and the attenuation of distracting objects, with applications in computational photography and habitat monitoring. Both analog and digital systolic VLSI circuit implementations are described with emphasis on recent progress using field programmable gate array (FPGA)-based and digital VLSI circuits that can potentially operate at radio frequencies in the multi-GHz range. These new innovations open up exciting possibilities for real-time MD filters having frames rates in the multi- $\mathrm{GHz}$ for emerging radio frequency (RF) antenna signal processing and imaging systems. 


\section{Introduction}

$\mathrm{M}$ ultidimensional (MD) signals are natural extensions of their one dimensional (1-D) counterparts, where the signals are measured and defined over more than one independent variable [1], [2]. Whereas 1-D signals are typically defined over time or space, a three dimensional (3-D) signal might, for example, be defined over two orthogonal $x y$ spatial dimensions and over the temporal dimension $t$, implying three independent variables (i.e., dimensions). The extension of 1-D signal processing techniques to the MD case is important because the domains of practical signals are inherently distributed over multiple dimensions and always over both 3-D space and 1-D time. Typically, important information exists within the spatio temporal domain of an MD signal that cannot be extracted from a corresponding 1-D signal, such as its directional or structural properties.

In this article, the basic principles of MD signal processing are reviewed and then exploited to describe some emerging 2-D, 3-D and 4-D applications, as illustrated in Fig. 1. We describe ways in which MD filtering may be employed in applications such as cognitive radio systems [3], [4], radio astronomy including realtime array processing of digitized RF cosmic signals [7], [8] and finally light field optical systems including depth filtering for digital photography [5], [6].

The fundamental challenge in MD filter design and implementation is the high complexity of the required hardware, which scales exponentially with the dimensionality of the filter. Therefore, not surprisingly, and in an attempt to reduce complexity, early work focused on the 2-D IIR case, for which it eventually became evident that simply extending the 1-D IIR approach (i.e., to similarly apply MD feedback within the filters) was fraught with stability problems. Typically, numerical design methods for achieving highly-directional fan-shaped or beam-shaped 2-D passbands yielded unstable inputoutput 2-D difference equations. There is extensive literature on this topic that is beyond the scope of this tutorial paper. Some of this work can be found in contributions such as [12]-[19] and references therein. Here, we describe both MD FIR and stable IIR filter design methods for achieving highly-directional MD passbands, drawing heavily on the work of the MD Signal Processing Group at the University of Calgary [20], the Advanced Signal Processing Circuits group at the University of Akron [21] and the Australian Centre for Field Robotics at the University of Sydney [22].
In Section II, we briefly review the fundamental theory of MD filter design. Excellent texts, such as [23], provide a thorough description of the topic. Here, we emphasize the use of the MD Laplace transform in filter design, which is not thoroughly treated in standard textbooks. Further, we emphasize the use of the first-order so-called "frequency-planar" MD transfer function as an important pseudo-passive stable IIR building block for making higher-order MD IIR filters.

In Section III and Section IV, some emerging potential applications of spatio-temporal 2-D and 3-D FIR filters are described. First, beamforming 2-D FIR asymmetric trapezoidal filters for cognitive radio systems and, second, beamforming 3-D FIR frustum filters for broadband transient detection systems in radio astronomy.

In Section V, we emphasize VLSI circuit implementations of real-time digital and analog circuits for 2-D and 3-D IIR spatio-temporal cases, where the ultimate objective is to make real-time beam formers with high directional-selectivity, high-throughput, low-latency and low-complexity. Recently published systolic architectures are described that are based on Frequency-Planar prototypes, and yield an especially fast throughput of One complete spatial Frame of data Per Clock Cycle (the so-called "OFPCC" method).

In Section VI, we describe novel approaches by which the 4-D signals from a light field camera have been enhanced using 4-D filters, offering enhanced imaging capabilities in challenging environments. Applications include imaging in low light, in turbid (murky) water, and in the presence of moving distractors. Motivating examples include long-term underwater habitat monitoring, wildlife photography, and marine archaeology on shipwrecks in the Aegean Sea. Finally, Section VII concludes the article.

\section{Fundamental Theory of 2-D, 3-D and 4-D Filter Design and Implementation}

In general, an MD signal $w(\mathbf{t})$ [1] has the real M-tuple domain $\mathbf{t}=\left(t_{1}, t_{2}, \ldots, t_{M}\right) \in \mathbb{R}^{M}$ and may be represented in the complex-frequency s-domain by the Laplace transform

$$
W(\mathbf{s})=\frac{1}{(2 \pi)^{M}} \int w(\mathbf{t}) e^{-\mathbf{s}^{\mathrm{T}} \mathbf{t}} d \mathbf{t},
$$

where we have written the complex M-tuple $\mathbf{s}=\left(s_{1}\right.$, $\left.s_{2}, \ldots, s_{M}\right) \in \mathbb{C}^{M}$ as the column matrix $\mathbf{s} \equiv\left[\begin{array}{ll}s_{1} & s_{2} \ldots s_{M}\end{array}\right]^{\mathrm{T}}$. The corresponding MD Fourier transform of $w(\mathbf{t})$ is $W(j \omega)$, where $\omega \equiv\left[\begin{array}{llll}\omega_{1} & \omega_{2} & \ldots & \omega_{M}\end{array}\right]^{\mathrm{T}}$ [23]. For simplicity,

Arjuna Madanayake and Chamith Wijenayake are with the Department of Electrical and Computer Engineering, University of Akron, Akron, OH, USA. Arjuna Madanayake, Thushara K. Gunaratne, and Leonard T. Bruton are with the Department Electrical and Computer Engineering, University of Calgary, Calgary, AB, Canada. Donald G. Dansereau and Stefan B. Williams are with the Department of Mechanical Engineering, University of Sydney, Sydney, Australia. E-mails: arjuna@uakron.edu or bruton@ucalgary.ca. 


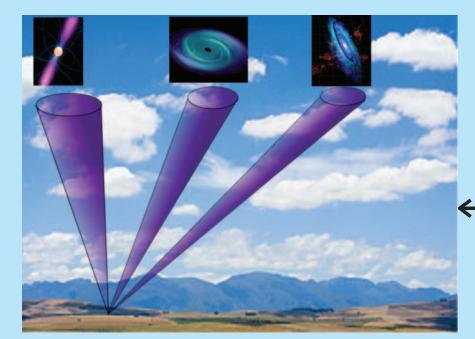

(a)

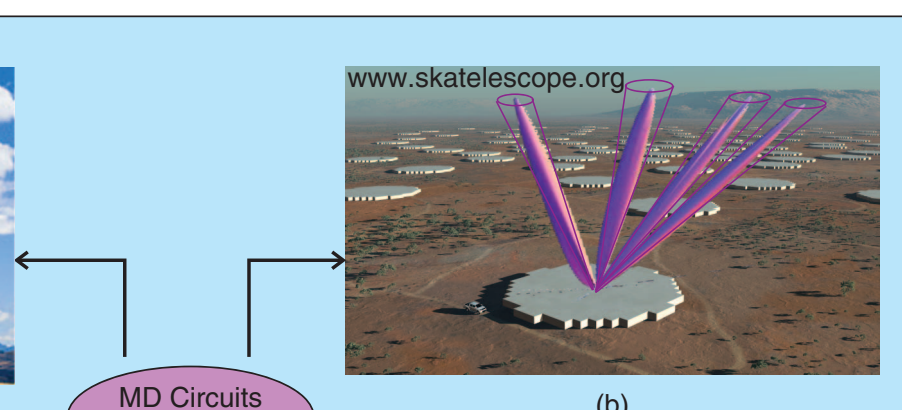

(b)

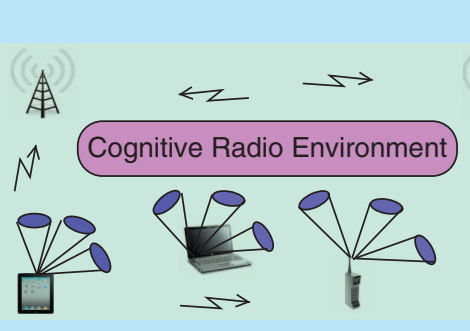

(c)

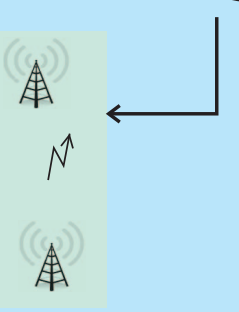

MD Circuits
and Systems

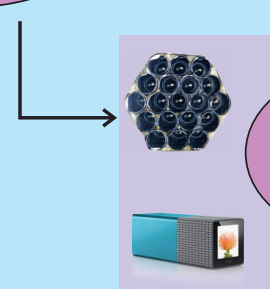

a

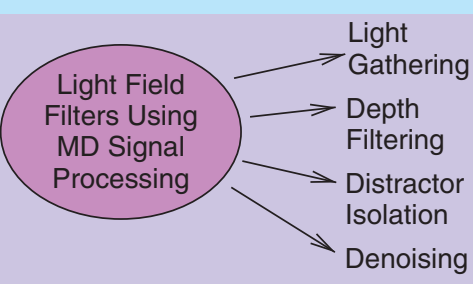

(d)

Figure 1. Emerging applications for FIR/IIR space time plane wave filters include aperture arrays for cosmic imaging applications such as radio astronomy and pulsar astrometry (a). A particularly interesting possibility for real-world engineering applications of high performance MD filters exist in the so-called Square Kilometer Array (SKA) project where multiple far-field beams are required from dense aperture arrays (b). Further, MD filters have been employed in front-end beamforming applications in cognitive radio environment (c) and light gathering, depth filtering, distractor isolation and denoising applications in light field signal processing (d).

we shall assume here and in Sections III, IV and V that the domain is 4-D-spatio-temporal as given by $(x, y, z, c t)$ where $t$ is time, $(x, y, z) \in \mathbb{R}^{M}$ is the 3-D Euclidean spatial coordinate and $c$ is a constant having the dimensions of speed (and is equal to the speed of light in Sections III, IV and V). Extensions to higher dimensions will be obvious. By analogy with the physical energy dissipated $\left(E=\int w^{2}(t) d t / R\right)$ in a $1 \Omega$ resistance element due to terminal voltage $w(t), E(\mathbf{t})$ of a real-valued MD signal $w(\mathbf{t})$ is defined as the following M-integral over the entire domain.

$$
E=\int_{\mathbf{t} \in \mathbb{R}^{M}} w(\mathbf{t})^{2} d \mathbf{t}
$$

From (2) and the MD version of the well-known Parseval relation, it may be shown that

$$
E=\int_{\omega \in \mathbb{R}^{M}} \psi(\omega) d \omega
$$

where $\psi(\boldsymbol{\omega})$ is the MD Energy-Density spectrum of $w(\mathbf{t})$ that relates to the corresponding MD Fourier transform such that

$$
\psi(\omega)=\frac{\left|W(j \omega)^{2}\right|}{(2 \pi)^{M}} .
$$

It is beyond the scope of this article to describe the full range of useful MD signals $w(\mathbf{t})$ and their corresponding energy density spectra $\psi(\omega)$, although the motivated reader may wish to consult a relevant MD textbook [23]. Such signals include 2-D lowpass and 2-D highpass pass signals in image processing and 2-D constant-velocity (i.e., linear-trajectory) spatio-temporal signals in video processing. In this article, we focus on ideal MD plane waves $w(\mathbf{t})$ and the corresponding practical MD signals that are obtained from ideal plane waves by means of multiplicative MD aperture functions (i.e., MD window functions) and by sampling them in some or all of their $\mathrm{M}$ dimensions. We shall pay special attention to 2-D/3-D spatio-temporal filters and their applications in beamforming and to the 4-D spatial filtering of light fields.

Using the 4-D spatio-temporal case for which $\mathbf{t} \equiv(x, y, z, c t) \in \mathbb{R}^{4}$, the fundamental 4-D relationship between the continuous-domain input signal $w_{a}(\mathbf{t})$ of a 4-D linear shift invariant (LSI) spatio-temporal filter and its resultant output signal $y_{a}(\mathbf{t})$ is given by the 4-D partial differential equation of order $\left(N_{x}, N_{y}, N_{z}, N_{c t}\right)$

$$
\begin{aligned}
& \sum_{m=0}^{N_{c t}} \sum_{l=0}^{N_{z}} \sum_{k=0}^{N_{y}} \sum_{j=0}^{N_{x}} p_{j k l m} \frac{\partial^{m}}{\partial(c t)} \frac{\partial^{l}}{\partial z} \frac{\partial^{k}}{\partial y} \frac{\partial^{j}}{\partial x}\left[w_{a}(\mathbf{t})\right] \\
& =\sum_{m=0}^{N_{c t}} \sum_{l=0}^{N_{z}} \sum_{k=0}^{N_{y}} \sum_{j=0}^{N_{x}} q_{j k l m} \frac{\partial^{m}}{\partial(c t)} \frac{\partial^{l}}{\partial z} \frac{\partial^{k}}{\partial y} \frac{\partial^{j}}{\partial x}\left[y_{a}(\mathbf{t})\right] .
\end{aligned}
$$




\section{Note that the spatial shifts $\Delta x, \Delta y$ and $\Delta z$ do not require specific hardware components because they correspond to spatially sampling signals at different spatial locations, whereas temporal shifts $\Delta T$ require hardware delay elements (such as analog delay lines for analog-MD filters or digital RAM for digital-MD filters).}

Note that, in this notation, $\partial^{0}\left[w_{a}(\mathbf{t})\right] / \partial t_{i}=w_{a}(\mathbf{t})$ and $q_{0000}=1$. The $\left(2 N_{z} N_{y} N_{x} N_{c t}-1\right)$ real constant coefficients $\left\{p_{j k l m}, q_{j k l m}\right\}$ are weighting terms that constrain the relationship between the input $w_{a}(\mathbf{t})$, the output $y_{a}(\mathbf{t})$ and the above partial derivatives. The $\left\{p_{j k l m}, q_{j k l m}\right\}$ are selected to approximate the desired input-output relationship, such as the desired shape of the MD passband (e.g., 2-D-fan, 3-D-cone, etc. [24] [25]).

Importantly, we require zero-valued 4-D initial conditions (ZICs): that is, the input $w_{a}(\mathbf{t})$ and all of its partial derivatives in (5), as well as all of the internal states of the filter, are zero-valued everywhere outside of the positive (i.e., +++ ) octant of $\mathbf{t} \in \mathbb{R}^{3}$. Then the 4-D Laplace transform of (5) yields

$$
\begin{aligned}
\sum_{m=0}^{N_{c t}} \sum_{l=0}^{N_{z}} \sum_{k=0}^{N_{y}} \sum_{j=0}^{N_{x}} p_{j k l m} s_{x}^{j} s_{y}^{k} s_{z}^{l} s_{c t}^{m} W_{a}(\mathbf{s}) \\
=\sum_{m=0}^{N_{c t}} \sum_{l=0}^{N_{z}} \sum_{k=0}^{N_{y}} \sum_{j=0}^{N_{x}} q_{j k l m} s_{x}^{j} s_{y}^{k} s_{z}^{l} s_{c t}^{m} Y_{a}(\mathbf{s}),
\end{aligned}
$$

which, after re-arrangement, gives the 4-D Laplace transform transfer function

$$
T(\mathbf{s}) \equiv \frac{Y_{a}(\mathbf{s})}{W_{a}(\mathbf{s})}=\sum_{m=0}^{N_{c t}} \sum_{l=0}^{N_{z}} \sum_{k=0}^{N_{z}} \sum_{j=0}^{N_{x}} \frac{p_{j k l m} s_{x}^{j} s_{y}^{k} s_{z}^{l} s_{c t}^{m}}{q_{j k l m} s_{x}^{j} s_{y}^{k} s_{z}^{l} s_{c t}^{m}}
$$

where the corresponding 4-D Fourier transform transfer function is $T(j \omega)=T\left(j \omega_{x}, j \omega_{y}, j \omega_{z}, j \omega_{c t}\right)$, implying that the input-output 4-D energy density spectra are related according to

$$
\psi_{Y}(\omega)=|T(j \omega)|^{2} \psi_{W}(\omega) .
$$

By properly selecting the coefficients of (7), we may shape the energy density spectrum in useful ways according to (8). This continuous-domain 4-D filtering operation has the complex-valued 4-D steady-state frequency response transfer function $T(j \omega)$, corresponding to the magnitude frequency response transfer function (i.e., gain) $|T(j \omega)|$.

\section{A. MD-Analog and MD-Digital Spatio-Temporal Filters}

In practice, the signals and the partial derivatives in (5) are not technologically realizable over spatiallycontinuous dimensions. However, subject to Nyquist spatial sampling constraints, these signals may be fully represented by temporally-continuous spatiallydiscrete samples, having mixed-domains of the form $\left(n_{x} \Delta x, n_{y} \Delta y, c t\right) \in \mathbb{N}^{2} \mathbb{R}$ (in the case of 3-D). Such mixeddomain versions of (5) belong to the class of MD-analog ST filters [26]-[33] having analog circuit realizations, typically using RC-active circuits. The signals in (5) may be both temporally-sampled and spatially-sampled, resulting in signals having fully-discrete-domains, such as $\left(n_{x} \Delta x, n_{y} \Delta y, c n_{t} \Delta T\right)$. The corresponding filters may be implemented as MD-analog filters: for example, by using the capacitive charges in switched-capacitor circuits to represent signal amplitudes. However, more typically, the amplitudes of fully-discrete-domain signals are represented in digital form, resulting in the more widely-used class of $M D$-digital filters, having non-trivial problems due to finite arithmetic, including nonlinear overflow and underflow phenomena (where the latter usually manifests itself as digital noise and thereby limits dynamic range).

\section{B. MD Signal Flow Graph (SFG) Decompositions}

After discretization of (5) in all four dimensions, the corresponding input-output relationship for the 4-D discrete-domain filter is given by the 4-D difference equation

$$
\begin{aligned}
& \sum_{m=0}^{N_{c t}} \sum_{l=0}^{N_{z}} \sum_{k=0}^{N_{y}} \sum_{j=0}^{N_{x}} a_{j k l m} w\left(n_{x}-j, n_{y}-k, n_{z}-l, n_{c t}-m\right) \\
& =\sum_{m=0}^{N_{c t}} \sum_{l=0}^{N_{z}} \sum_{k=0}^{N_{y}} \sum_{j=0}^{N_{x}} b_{j k l m} y\left(n_{x}-j, n_{y}-k, n_{z}-l, n_{c t}-m\right) .
\end{aligned}
$$

Note that

$$
w\left(n_{x}, n_{y}, n_{z}, n_{c t}\right) \equiv w_{a}\left(n_{x} \Delta x, n_{y} \Delta y, n_{z} \Delta z, n_{c t} \Delta T\right),
$$

and

$$
y\left(n_{x}, n_{y}, n_{z}, n_{c t}\right) \equiv Y_{a}\left(n_{x} \Delta x, n_{y} \Delta y, n_{z} \Delta z, n_{c t} \Delta T\right) .
$$

The coefficients $\left\{a_{j k l m}, b_{j k l m}\right\}$ are the discrete-domain filter design parameters. Note that the spatial shifts $\Delta x, \Delta y$ and $\Delta z$ do not require specific hardware components because they correspond to spatially sampling signals at different spatial locations, whereas temporal shifts $\Delta T$ require hardware delay elements (such as analog delay lines for analog-MD filters or digital RAM for digital-MD filters). In the spatio-temporal 4-D-digital case, 


\section{As an extreme but nevertheless practical example, a typical 3-D FIR beam transfer function of order $(20,20,20)$ has $20^{3}-1=7,999$ independent multiplier coefficients. However, an equally selective 3-D IIR beam filter can be realized using two cascaded 3-D first-order IIR frequency planar filters, each requiring only 15 multipliers.}

for example, the 4-D SFGs are realized using interconnections of digital multipliers and adders, time delays $\Delta T$ and spatial shifts $\Delta x, \Delta y$ and $\Delta z$. The most widely employed MD discrete-domain SFG decompositions are the direct-form [23], the differentiator-form [28], [34]-[37] and the discrete-integrator form [38] [35]. The choice of SFG decomposition can significantly affect nonideal performance such as hardware complexity, sensitivity of the shape of the MD passband and stopband to multiplier coefficient quantization and dynamic range.

Taking the 4-D z-transform of (9) yields the corresponding discrete-domain transform transfer function

$$
\begin{aligned}
H\left(z_{x}, z_{y}, z_{z}, z_{c t}\right) & \equiv \frac{Y\left(z_{x}, z_{y}, z_{z}, z_{c t}\right)}{W\left(z_{x}, z_{y}, z_{z}, z_{c t}\right)} \\
& =\sum_{m=0}^{N_{c t}-1} \sum_{l=0}^{N_{z}-1} \sum_{k=0}^{N_{y}-1} \sum_{j=0}^{N_{x}-1} \frac{a_{j k l m} z_{x}^{-j} z_{y}^{-k} z_{z}^{-l} z_{c t}^{-m}}{b_{j k l m} z_{x}^{-j} z_{y}^{-k} z_{z}^{-l} z_{c t}^{-m}}
\end{aligned}
$$

where $b_{0000}=1$. Equation (10) is the 4-D z-transform transfer function where the corresponding 4-D frequency response transfer function $H\left(e^{j \omega_{c t}}, e^{j \omega_{x}}, e^{j \omega_{y}}, e^{j \omega_{z}}\right)$ is written for brevity as $H\left(e^{j \omega}\right)$. In this 4-D case, the discrete-domain approximation problem is to find a set of coefficients $\left\{a_{j k l m}, b_{j k l m}\right\}$ that yields a $4-\mathrm{D}$ gain function $\left|H\left(e^{j \omega}\right)\right|$ that approximates the desired passband shape while retaining the internal stability of the states of the filter. The complexity of the final design scales approximately with $N_{x} N_{y} N_{z} N_{c t}$, which has significantly limited practical applications to low-order transfer functions.

\section{On Approximation and Complexity}

Approximation is the process of finding the values of the coefficients of (10) that yield a useful relationship between the input and output signals. For example, by shaping the energy density spectrum of the input signal according to (8) so that $|T(j \omega)|$ has a 4-D hypercone-shaped passband. For MD FIR case, approximation by means of numerical optimization of the coefficients may be employed. However, for the MD IIR case, the stability of (9) is a major challenge because the necessary and sufficient conditions for the feedback coefficients $\left\{b_{j k l m}\right\}$ to yield a stable filter are not known for the general case. In the MD-IIR case with $M>1$, numerically optimizing $\left\{a_{j k l m}, b_{j k l m}\right\}$ so that $|T(j \omega)|$ approximates the desired shape of the passband invariably yields an unstable $M D$ difference equation for which stabilization strategies are not generally available. Further, and as in the 1-D case, MD-IIR filters require transfer functions of much lower order than approximately-equivalent MD-FIR transfer functions, implying significantly less hardware. This is a major incentive to solve the stability problems associated with (9). As an extreme but nevertheless practical example, a typical 3-D FIR beam transfer function of order $(20,20,20)$ has $20^{3}-1=7,999$ independent multiplier coefficients in (9). However, an equally selective 3-D IIR beam filter can be realized using two cascaded 3-D firstorder IIR frequency planar filters [39], each requiring only 15 multipliers, representing a potential reduction in complexity that exceeds two orders of magnitude. We suggest that the high complexity of MD FIR filters has impeded their widespread application and that MD IIR filters can alleviate this disadvantage.

\section{Stability Using MD Pseudo-Passive Prototypes}

As in the 1-D case, the inverse transform of (10) is the MD unit impulse response $h(\mathbf{n})$ and it can be shown that the internal stability of (9), under zero initial conditions, is assured if $h(\mathbf{n})$ is absolutely-summable over $\mathbf{n} \in \mathbb{Z}^{4}$ [23]. Unfortunately, with the exception of MD FIR and trivial MD IIR cases, algebraic relationships between the IIR function $h(\mathbf{n})$ and the coefficients of (9) are usually unknown or very complicated [40]. Nevertheless, designers of MD-IIR filters have effectively solved the stability problem [41]-[44] by obtaining the coefficients $\left\{a_{j k l m}, b_{j k l m}\right\}$ of (9) by employing a suitable continuous-time partial differential input-output transfer function belonging to a pseudo-passive prototype network. In particular, MD inductor-capacitor-resistor (LRC) prototype networks (which are interconnections of resistance, MD-inductance and MD-capacitance elements) are a rich source of such transfer functions and are (for properly designed discrete-domain realizations) both pseudo-passive and stable for non-negative values of the MD prototype circuit elements. Fig. 2(a) shows a pseudo-passive first-order spatio-temporal self-resonant 3-D LR prototype network. This network forms an important IIR building block for higher-order designs, as described in this contribution. The full exploration and exploitation of such networks remains a fruitful research topic. Pseudo-passive networks have 


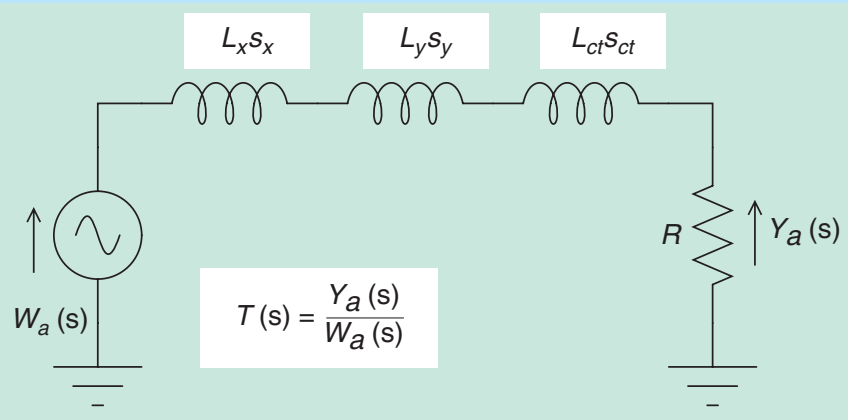

(a)

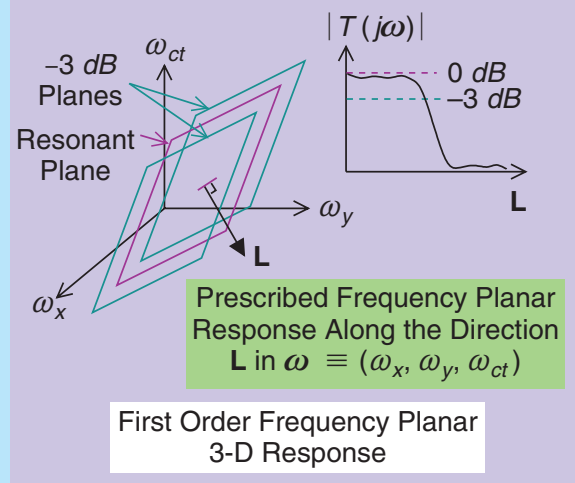

(b)

Figure 2. A first order 3-D inductor-resistor pseudo passive prototype network (a) and the corresponding frequency planar response (b).

been reported for $2-\mathrm{D} / 3-\mathrm{D}$ beamforming and 3 -D video processing [35]-[37], [45], [46].

\section{E. The Self-Resonant Frequency \\ Planar Building Block}

The first-order 3-D self-resonant prototype network [Fig. 2(a)] having a frequency planar response as shown in Fig. 2(b) was first used for the enhancement of 3-D objects in video [47]-[53] and is also a powerful building block for the realization of high-performance 3-D beam and cone filters [39]. By definition, the 3 -D series inductances $L_{i}, \quad i=1,2,3$ satisfy the relationships $w_{j}(\mathbf{t})=L_{j} \partial[i(\mathbf{t})] / \partial t_{j}, j=1,2,3$. Then, employing Kirchoff's voltage-current laws we obtain $w_{a}(\mathbf{t})+\sum_{j=1}^{3} \frac{\partial}{\partial t_{j}}\left[w_{a}(\mathbf{t})\right]=y_{a}(\mathbf{t})$. Taking the 3-D Laplace transform of this equation, and rearranging yields the 3 -D transfer function $T(\mathbf{s})=\frac{1}{1+\mathbf{s}^{T} \mathbf{L}}$, which is known as the 3 -D frequency planar transfer function of order one. The complex steady-state 3-D frequency response function is found by setting $s_{j}=j \omega_{j}$ giving $T(j \omega)=\frac{1}{1+j \omega^{T} \mathbf{L}}$. Selecting $\|\mathbf{L}\|_{2}>>1$, the magnitude frequency response $|T(j \omega)|$ closely surrounds the 3 -D passband plane $\omega^{T} \mathbf{L}=0$. A review of MD plane waves can be found in the Appendix. This plane passes through the origin with direction given by the unit normals $\pm \frac{\mathbf{L}}{\|L\|_{2}}$.

Note that, $|T(j \omega)|$ is unity on this plane, which is at the centre of the 3-D frequency-planar passband. The inductances are self-resonant in the plane $\omega^{T} \mathbf{L}=0$ [51], and the shape of $|T(j \omega)|$ rapidly decreases outside of the plane with a perpendicular bandwidth given by $1 /\|\mathbf{L}\|_{2}$ as shown in Fig. 2(b). In summary, the first-order frequencyplanar MD relation yields an MD frequency response that surrounds an MD plane with selectivity and direction that may be independently determined by selection of the MD inductance elements of $\mathbf{L}$.
Later in this article, we describe how the transform transfer function of the MD network may be employed as a building block for MD plane wave filtering. By cascading frequency-planar filters to form beam filters, and by employing them in 2-D, 3-D and 4-D polyphase filter banks, it has been shown that high-performance low-complexity highly-directional plane wave filters may be realized [54]-[59]. It was proposed in [45] that discrete domain versions of the inductance-resistance filter can be implemented using pseudo-passive MD wave-digital filters (WDFs), leading to a number of real-time implementations [56], [60], [61]. However, the approach described here is to directly discretize the voltage-current variables of the underlying MD passive network.

Stability in the discrete-domain is assured by applying the MD bilinear transformation (BLT) $s_{i}=\frac{2}{\Delta_{i}} \cdot\left(1-z_{i}^{-1}\right) /\left(1+z_{i}^{-1}\right) \quad i=1,2, \ldots, M$, to $\mathrm{s}$-domain transfer functions of the MD passive networks, where $\Delta_{i}$ is the inter-sample distance or inter-sample time. There exist many different signal flow graph architectures (that is, decompositions) for the computation of any given input-output MD difference equation. In the following, we determine the coefficients of the MD difference equation and then obtain the filter coefficients by means of the MD BLT.

\section{F. Employing the Frequency Planar Building Block}

There is a wealth of literature on the design and implementation of linear shift-invariant (LSI) 2-D FIR and IIR filters having lowpass or highpass passbands, typically for smoothing or edge-enhancement applications in image or video processing. Here, we focus on the passband shapes required for directionally selective filtering of broadband plane waves in 


\section{The selective filtering of MD plane waves involves the design of MD filters having MD passbands that closely surround (all or part of) the MD line of the ROS over the entire frequency range of interest.}

2-D, 3-D and 4-D. We employ simple first-order 3-D or 4-D self-resonant pseudo-passive continuous-domain prototype filters as the basic building block. This building-block has been used extensively for realizing low order, low complexity, highly selective 2-D, 3-D and 4-D filters.

\section{G. Directional Filtering of Ideal Broadband Plane Waves}

We consider here the region of support (ROS) of $W_{a}(j \omega)$ and thereby $\Psi(\omega)$ for a spatio temporal 4-D plane wave having direction vector $\left[d_{x} d_{y} d_{z} 1\right]^{T}$, it may be shown that the corresponding 4-D Fourier transform $W_{a}(j \omega)$ and energy density spectrum $\Psi(\omega)$ have the 4-D ROS in the frequency domain that is confined to the straight line

$$
\frac{\omega_{x}}{d_{x}}=\frac{\omega_{y}}{d_{y}}=\frac{\omega_{z}}{d_{z}}=\omega_{c t} .
$$

This 4-D line passes through the origin with an orientation in 4-D frequency space $\omega \in \mathbb{R}^{4}$ that is given by the direction of the plane wave $w_{a}(\mathbf{t})$ in $\mathbf{t} \in \mathbb{R}^{4}$.

For this reason and in general, the selective filtering of MD plane waves involves the design of MD filters having MD passbands that closely surround (all or part of) the MD line of the ROS over the entire frequency range of interest. Included in the shapes of such ideal MD passbands are 2-D pencil-shapes (i.e., beam-shapes), dual-cone-shapes and dual-frustum shapes. In 2-D, the corresponding passband shapes are 2-D beams, dualfans and dual-trapezoids. Similarly, in 4-D, the corresponding pass band shapes are dual hyper-pencils, dual-hyper-fans and dual-hyper-cones.

\section{H. Discrete Realizations of Beam, Fan and Cone Filters}

By applying the MD bilinear transformation to the MD Laplace transfer function of pseudo-passive inductor-resistor frequency planar building block transfer functions, we retain practical-BIBO stability and structural stability of the resultant MD discrete domain filter. The penalty that is paid for retaining stability in this way is that the continuous domain MD passband undergoes MD tiling and warping, due to the MD BLT. The obvious solution is to oversample the signal in all $\mathrm{M}$ dimensions, although this is expensive in the spatial domain because the number of sensors and data converters scales linearly with the number of spatial samples. Methods of Nyquist undersampling have been proposed [24] and exploited for reducing the complexity of narrow $\mathrm{MD}$ beam, fan and cone filters.

\section{MD IIR Synthesis, Design and Implementation}

The discovery of a stable decomposition for MD polynomials [42] has, over the last several decades, led to a variety of methods for realizing stable MD IIR filters. By design, such stable polynomials form the denominator of the MD Laplace transform transfer function and thereby yield stable transfer functions. Pseudo passive MD resistively terminated passive filters and wavedigital filters are especially useful classes of MD filters [62], [63]. The self-resonant first-order MD frequency planar transfer function having frequency planar response as shown in Fig. 2(b) is perhaps the most simple and useful example.

Using MD pseudo passive prototypes, real-time 2-D and 3-D IIR filter instruments have been reported. It has been shown that 3-D frequency planar IIR filters can be used to recover and track invisible objects in very noisy television image sequences [50]-[53] and also to track vehicles in traffic videos [46]. In order to improve throughput, systolic implementations were shown to be possible [25], [64]-[67] and, as VLSI circuits have improved, high frequency systolic operation has been shown to be feasible, including 4-D filter banks [68].

MD-Filter Banks have been proposed using intraband MD beam filters, often using the polyphase decomposition to realize 2-D fan filters, 3-D cone filters and 4-D hyper-fan filters [54], [58]-[60], [68]. MD polyphase filter banks are of low-complexity and arbitrarily-narrow directional selectivity with an overall performance that is primarily limited by the unavoidable finite aperture (i.e., windowing) effects, the need to avoid MD-aliasing by sampling above the Nyquist rate and the need for adequate arithmetical precision. For low frequency applications, significant reductions in the hardware requirements for MD filters can be achieved by temporally-scanning using a single analog to digital converter (ADC) across multiple spatial samples of the input $w_{a}(\mathbf{t})$ [28]. In the following four sections, 


\section{The real-time adaptive broadband-bandpass beamforming methods to be used in cognitive radio systems must accommodate the gradual time-variation of the DOAs of the desired signals as well as the instantaneous changes of the operating frequency band and the bandwidth.}

some specific applications of MD filters are described in more detail.

\section{Adaptive 2-D FIR Beamforming in Cognitive Radio Systems}

In the Unites States, the Federal Communications Commission (FCC) licenses the electromagnetic spectrum based on carrier frequency and bandwidth requirement to primary users. These primary users in turn operate in their licensed band to provide services such as wireless communications, radar and habitat monitoring. However, explosive growth in wireless networks is causing spectrum scarcity for emerging broadband access needs because the demand for spectrum far exceeds the licensed bands available for communications. The problem is further complicated by the fact that most licensed users do not utilize their spectral bands most of the time, leading to spectral white spaces, which are essentially relatively unused licensed spectral bands that may potentially be employed for communications by non-licensed (secondary) users when the primary user is not transmitting in that band [3], [4], [9].

Cognitive radio systems exploit dynamic allocation of spectral recourses such as operational frequency band, the signal bandwidth, power transmission and wireless modulation techniques in order to facilitate a broad range of wireless communication and entertainment applications [70]-[72]. Software defined radio (SDR) architectures have been proposed for the front-end processing of cognitive radio systems that exploit both dynamic allocation of the radio-spectrum and power management [70], [71], [73]. Real-time spectral sensing detects available frequency channels and the brain of cognitive radio systems, the control system assigns the spectral recourses such that the data throughput is maximized [70], [72]. Using smart antennas for the adaptive beamforming, cognitive radio systems effectively allocate the spectrum and efficiently control the signal power in such a way as to reduce interference and therefore achieve maximum throughput in data transfer [70], [74], [75]. Typically, cognitive radio systems span several designated frequency-bands and inherently employ temporally-broadband-bandpass signals [70]-[72], [74], [75]. The real-time adaptive broadbandbandpass beamforming methods to be used in cognitive radio systems must accommodate the gradual timevariation of the DOAs of the desired signals [74], [75] as well as the instantaneous changes of the operating frequency band and the bandwidth [70], [72].

\section{A. ROS of Space-Time Cognitive Radio Environment}

For cognitive radio systems, for most of the time the receiver is in the far-field region of the transmitter. Hence, the simplified spatio-temporal plane wave propagation model [76] may be assumed for the signals received by the 1-D uniform linear array (ULA) in the broadband-bandpass-beamformer of the cognitive radio system. It has been shown in [77] that most previously proposed broadband-beamforming methods, employing either frequency-domain or time-domain methods, are either not sufficiently responsive to be adaptive in real-time, not sufficiently accurate or not cost effective with current technology. In [77], an adaptive discretedomain method is proposed for the beamforming of temporally-broadband-bandpass spatio-temporal plane wave signals in the context of a cognitive radio system having an SDR front-end.

Fig. 3 provides an overview of a typical ULA processing scheme employing 2-D spatio-temporal filters and broadband antenna elements such as Vivaldi antennas. The underlying 2-D spatio temporal filter can be either FIR or IIR in nature. Furthermore, the 2-D filter of interest can be realized in either 2-D spatio-temporal discrete domain (i.e., digital) having transfer functions of the form $H\left(z_{x}, z_{c t}\right)$ or 2-D discrete-space-continuoustime domain (i.e., analog) having transfer functions of the form $H_{m}\left(z_{x}, s_{c t}\right)$. In this section, we focus our attention to 2-D digital FIR filters having trapezoidal shaped passband, applicable to cognitive radio environments. In section $\mathrm{V}$ we briefly discuss several other types of 2-D filters including IIR types.

For the proposed 2-D FIR trapezoidal filter-based beamforming method, the pre-beamformer processing applied at the front-end of the cognitive radio system is illustrated by the schematics of Type-2: X-block in Fig. 3. As shown there the outputs of ULA elements are amplified by low noise amplifiers (LNAs), modulated by tunable local oscillators and then lowpass filtered to remove the highpass images and finally, synchronously-sampled using software tunable analog to digital converters, 


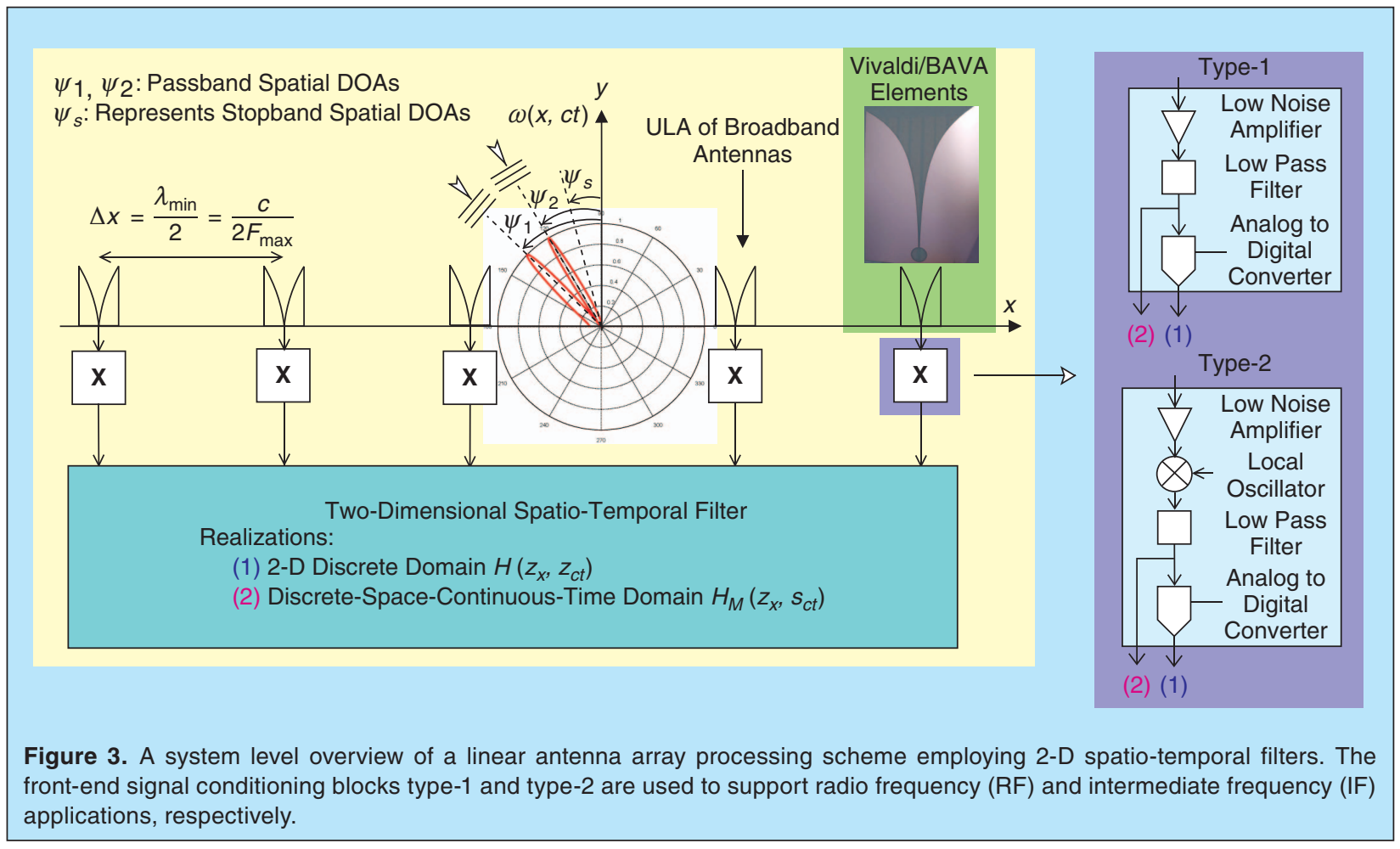

thereby yielding a complex-valued 2-D spatio-temporal sample sequence. Then, the above 2-D space-time sampled sequence is processed using a beamforming complex-coefficient 2-D FIR filter $H\left(z_{x}, z_{c t}\right)$ (see Fig. 4), having an asymmetric trapezoidal-shaped passband.

In [77], it is assumed that cognitive radio systems employ temporally-broadband-bandpass signals. Consider a real-valued temporally-bandpass wavefront function

$w(c t)$ having an instantaneous-bandwidth $\Omega_{\mathrm{I}}$ and an instantaneous center frequency $f_{\mathrm{IC}}$. According to [77], the relative bandwidth of a temporally-bandpass signal is characterized by its instantaneous bandwidth spread factor $K_{\mathrm{I}}$, defined as $K_{\mathrm{I}} \equiv \Omega_{\mathrm{I}} / 2 f_{\mathrm{IM}}$, where $f_{\mathrm{IM}}$ is the instantaneous maximum temporal frequency of $w(c t)$. According to [77], if a temporally-bandpass signal $w(c t)$ has $K_{\mathrm{I}}>0.1$, then it is considered to be a temporally-broadband-bandpass

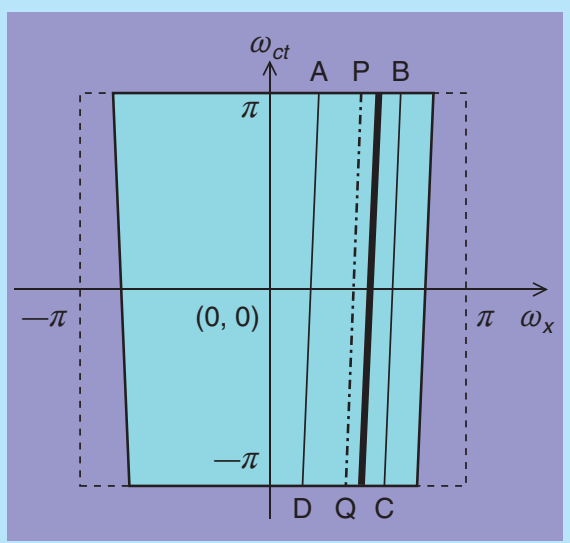

(a)

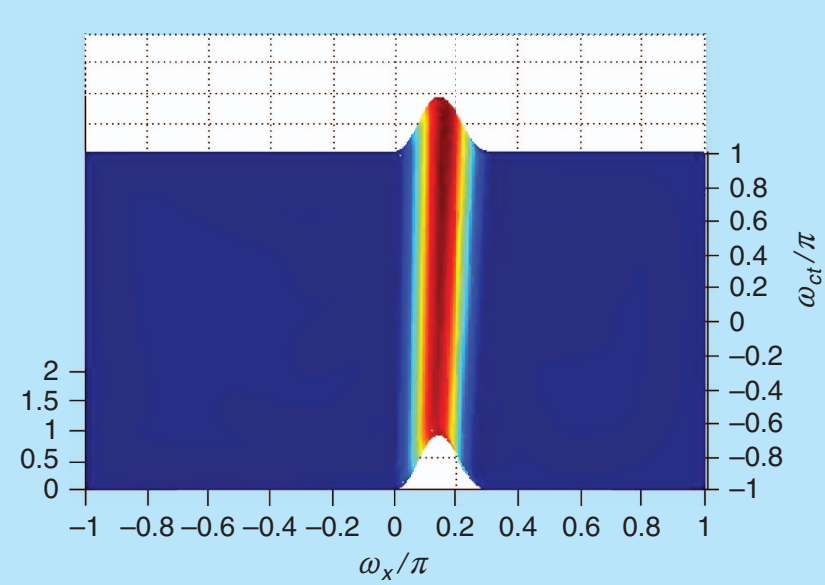

(b)

Figure 4. The ideal $R O S(P Q)$ of the spectra of space-time sampled plane wave and the ideal asymmetric trapezoidal shaped passband $(A B C D)$ that facilitates uniform angular selectivity (a). An example of the magnitude frequency transfer function of a 2-D FIR trapezoidal filter of order $(40 \times 32)(b)$. 


\section{The proposed ideal trapezoidal passband facilitates uniform angular selectivity and recovers the desired signal with minimum distortion and interference \\ provided that the ROSs of the spectra of the co-channel interfering signals lie outside this passband.}

signal. Also according to [77], the utilization of the spectrum by the cognitive radio system is characterized by the instantaneous system utilization factor $L_{\mathrm{I}}$, defined as $L_{\mathrm{I}} \equiv f_{\mathrm{IM}} / f_{\mathrm{SM}}$, where $f_{\mathrm{SM}}$ is the upper limit of the operational bandwidth of the cognitive radio system of interest.

In [77], it has been derived that ideally the ROS of the spectra of a space-time sampled sequence that corresponds to a propagating spatio-temporal plane wave arriving at the ULA with the azimuth angle $\psi_{0}$ (see Fig. 3) is given by

$$
\omega_{x}=\left(K_{\mathrm{I}} \omega_{t}+\left(L_{\mathrm{I}}-K_{\mathrm{I}}\right) \pi\right) \sin \left(\psi_{0}\right),
$$

which is shown by the line segment PQ in Fig. 4(a) inside the Principal Nyquist square $\left\{\left|\omega_{x} ; \omega_{t}\right| \leq \pi\right\}$. Therefore, the shaded region in Fig. 4(a) illustrates the range for all ROSs of the spectra of space-time sampled spatio-temporal plane waves arriving at the ULA with the azimuth angles $\psi \in\left[-90^{\circ}, 90^{\circ}\right]$.

\section{B. Broadband Bandpass Beamformer Design}

In [77], a discrete-domain 2-D spatio-temporal FIR filter having an asymmetric trapezoidal shaped-passband has been proposed to selectively enhance the spectral components of the desired signal, which are distributed on the region PQ and suppresses the spectral components of the co-channel interference signals and system noise, which are distributed on the remaining region inside of $\left\{\left|\omega_{x} ; \omega_{t}\right| \leq \pi\right\}$ [see Fig. 4(a)]. There is generally an uncertainty $\Delta \psi$ associated with the estimated DOA angle $\hat{\psi}_{0}$ of the propagating plane wave associated with the desired subscriber signal, implying that the actual DOA angle $\psi_{0}$ lies in the range $\psi_{0}=\left[\hat{\psi}_{0}-\Delta \psi, \hat{\psi}_{0}+\Delta \psi\right]$. This implies that the range of the ROS of the desired signal lies inside the hatched asymmetric trapezoidal region that is bounded by the straight lines

$$
\begin{aligned}
& \mathrm{AD}-\omega_{x}=\left(K_{\mathrm{I}} \omega_{t}+\left(L_{\mathrm{I}}-K_{\mathrm{I}}\right) \pi\right) \sin \left(\hat{\psi}_{0}-\Delta \psi\right) \\
& \mathrm{BC}-\omega_{x}=\left(K_{\mathrm{I}} \omega_{t}+\left(L_{\mathrm{I}}-K_{\mathrm{I}}\right) \pi\right) \sin \left(\hat{\psi}_{0}+\Delta \psi\right)
\end{aligned}
$$

and lie inside $\left\{\left|\omega_{x} ; \omega_{t}\right| \leq \pi\right\}$ as shown in Fig. 4(a). Therefore, irrespective of small errors between the actual and estimated DOA angles $\psi_{0}$ and $\hat{\psi}_{0}$, the proposed ideal trapezoidal passband facilitates uniform angular selectivity and recovers the desired signal with minimum distortion and interference provided that the ROSs of the spectra of the co-channel interfering signals lie outside this passband.

The unit impulse response $\tilde{h}\left(n_{x}, n_{c t}\right)$, of the ideal asymmetric trapezoidal shaped passband is given by the following closed-form algebraic expressions [77]. Note, $a \triangleq \sin \left(\hat{\psi}_{0}-\Delta \psi\right)$ and $b \triangleq \sin \left(\hat{\psi}_{0}+\Delta \psi\right)$.

For $n_{x} \neq 0,\left(a K_{\mathrm{I}} n_{x}+n_{c t}\right) \neq 0$ and $\left(b K_{\mathrm{I}} n_{x}+n_{c t}\right) \neq 0$,

$$
\begin{aligned}
\tilde{h}\left(n_{x}, n_{c t}\right)= & \frac{\sin \left(\pi\left(b K_{\mathrm{I}} n_{x}+n_{c t}\right)\right) e^{j \pi\left(L_{\mathrm{I}}-K_{\mathrm{I}}\right) b n_{x}}}{j 2 \pi^{2} n_{x}\left(b K_{\mathrm{I}} n_{x}+n_{c t}\right)} \\
& -\frac{\sin \left(\pi\left(a K_{\mathrm{I}} n_{x}+n_{c t}\right)\right) e^{j \pi\left(L_{\mathrm{I}}-K_{\mathrm{I}}\right) a n_{x}}}{j 2 \pi^{2} n_{x}\left(a K_{\mathrm{I}} n_{x}+n_{c t}\right)} .
\end{aligned}
$$

The special cases:

For $n_{x}=0$ and $n_{c t}=0$,

$$
\tilde{h}(0,0)=\left(L_{\mathrm{I}}-K_{\mathrm{I}}\right)(b-a) / 2 .
$$

For $n_{x}=0$ and $n_{c t} \neq 0$,

$$
\tilde{h}\left(0, n_{c t}\right)=K_{\mathrm{I}}(b-a)(-1)^{n_{c t}} /\left(j 2 \pi n_{c t}\right) .
$$

For $n_{x} \neq 0,\left(a K_{\mathrm{I}} n_{x}+n_{c t}\right) \neq 0$ and $\left(b K_{\mathrm{I}} n_{x}+n_{c t}\right)=0$,

$$
\begin{aligned}
\tilde{h}\left(n_{x}, n_{c t}\right)= & \frac{\sin \left(\pi\left(b K_{\mathrm{I}} n_{x}+n_{c t}\right)\right) e^{j \pi\left(L_{\mathrm{L}}-K_{\mathrm{I}}\right) b n_{x}}}{j 2 \pi^{2} n_{x}\left(b K_{\mathrm{I}} n_{x}+n_{c t}\right)} \\
& -\frac{e^{j \pi\left(L_{\mathrm{L}}-K_{1}\right) a n_{x}}}{j 2 \pi n_{x}} .
\end{aligned}
$$

For $n_{x} \neq 0,\left(a K_{\mathrm{I}} n_{x}+n_{c t}\right)=0$ and $\left(b K_{\mathrm{I}} n_{x}+n_{c t}\right) \neq 0$,

$$
\begin{aligned}
\tilde{h}\left(n_{x}, n_{c t}\right)= & \frac{e^{j \pi\left(L_{1}-K_{1}\right) b n_{x}}}{j 2 \pi n_{x}} \\
& -\frac{\sin \left(\pi\left(a K_{\mathrm{I}} n_{x}+n_{c t}\right)\right) e^{j \pi\left(L_{1}-K_{1}\right) a n_{x}}}{j 2 \pi^{2} n_{x}\left(a K_{\mathrm{I}} n_{x}+n_{c t}\right)} .
\end{aligned}
$$

For $n_{x} \neq 0,\left(a K_{\mathrm{I}} n_{x}+n_{c t}\right)=0$ and $\left(b K_{\mathrm{I}} n_{x}+n_{c t}\right)=0$,

$$
\tilde{h}\left(n_{x}, n_{c t}\right)=\frac{e^{j \pi\left(L_{1}-K\right) b n_{x}}-e^{j \pi\left(L_{1}-K\right) a n_{x}}}{j 2 \pi n_{c t}} .
$$

In [77], 2-D Hamming window function [78] $\operatorname{ham}_{2 \mathrm{D}}\left(n_{x}, n_{c t}\right)$ of size $\left(2 N_{x}+1\right) \times\left(2 N_{c t}+1\right)$ has been employed to determine the temporally-causal 2-D unit impulse response $\tilde{h}_{\mathrm{T}}\left(n_{x}, n_{c t}\right)$ of order $\left(2 N_{x} \times 2 N_{c t}\right)$

$$
\tilde{h}_{\mathrm{T}}\left(n_{x}, n_{t}+N_{c t}\right) \triangleq \operatorname{ham}_{2-\mathrm{D}}\left(n_{x}, n_{c t}\right) \tilde{h}\left(n_{x}, n_{c t}\right) .
$$




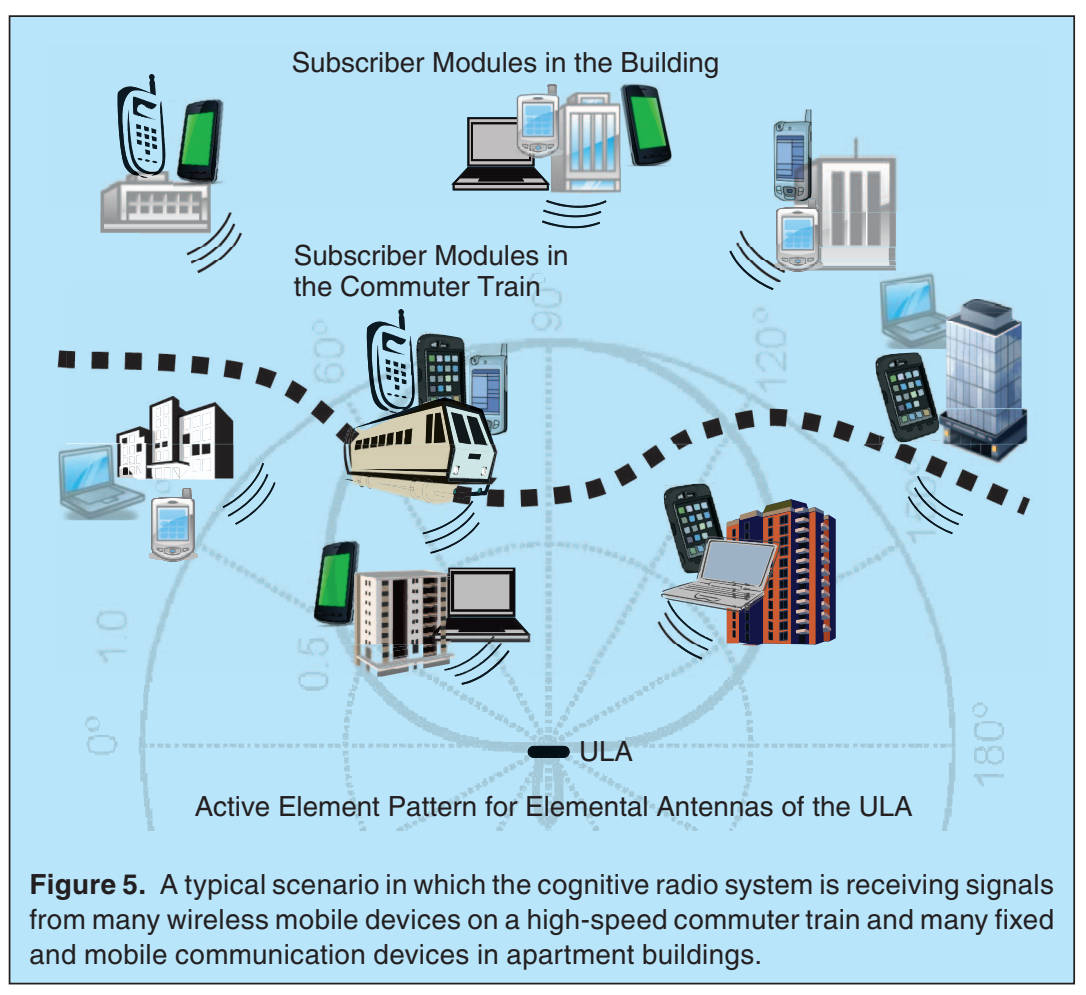

\section{An Example Application} of Adaptive 2-D FIR Beamforming

In order to provide an optimal quality of service for various communication applications, the cognitive radio system must dynamically allocate the operational frequency bands, the bandwidths and the signal power for the subscribed communication devices [70]-[72]. Hence, the beamformer should be able to adapt accordingly by changing the operational frequency-band and the beam-direction while maintaining the SIRs of the recovered desired signals so that they are above minimum threshold levels. A typical scenario considered in [77] had assumed the deployment of the proposed beamforming 2-D FIR trapezoidal filter as an adaptive broadband-bandpassbeamformer in a receiving arm of a cognitive radio system. Given the reciprocity between the signals in An example of the magnitude frequency transfer function of the 2-D FIR trapezoidal filter of order $(40 \times 32)$ that has been designed to meet the specifications $\hat{\psi}_{0}=20^{\circ}$, $\Delta \psi=5^{\circ}, L_{\mathrm{I}}=0.9$ and $K_{\mathrm{I}}=0.1$, is shown in Fig. 4(b).

the receiving and transmitting arms of the front-end of the SDR [79, ch. 4], the reciprocated application of the proposed adaptive 2-D FIR trapezoidal filter-based beamformer may also be used for beamforming within the transmission arms of a cognitive radio system.

The setup for a typical

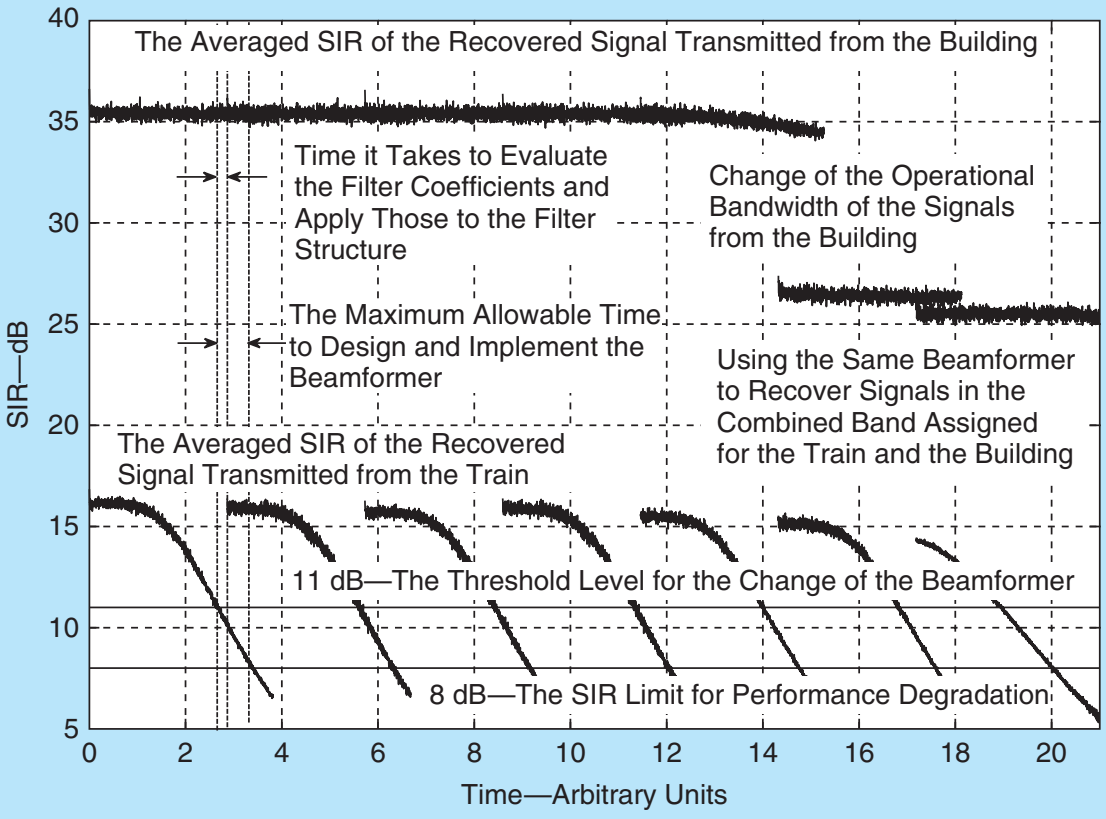

Figure 6. The averaged signal to interference ratio (in $\mathrm{dB}$ ) of the recovered signals that are transmitted from the commuter train and the apartment building as the train travels along the tracks. scenario where the proposed 2-D FIR asymmetric filter-based beamformers are used in recovering two groups of subscriber signals transmitted from subscriber modules in a high speed commuter train and subscriber modules in a building adjacent to the train tracks is shown in Fig. 5. A Monte-Carlo simulation has been conducted in order to estimate the average signal to interference ratio (SIR) at the two outputs of the two beamforming filters as the train travels along the tracks. As shown in Fig. 6, the average SIR for the signals transmitted from the train rapidly 
drops as the train moves. Thus, in order to maintain the average SIR levels above minimum threshold, the passband of the beamforming filters has to be adapted such that the receiving beam tracks the train. Further according to Fig. 6, the reconfiguration time for the proposed beamforming filter is small enough to maintain the SIR level sufficiently above the minimum threshold level. Using these Monte-Carlo simulations it has been demonstrated that the proposed 2-D FIR asymmetric trapezoidal filter-based beamformer achieves the best overall performance with respect to instantaneous adaptability, lowest distortion of the desired signal and highest attenuation of the cochannel interference signals for a given level of computational complexity [77].

\section{3-D FIR Frustum Filters for Broadband Beamforming of Focal Plane Array (FPA) Signals in Radio Astronomy}

\section{A. An Introduction to Broadband Beamforming of FPA Signals in Radio Astronomy}

Processing of cosmic radiation for subsequent extraction of celestial properties is of fundamental importance to the scientific community. Signal processing for radio astronomy is often about extracting 3-D cosmic spatio-temporal photonic plane waves from the overwhelming levels of sky-noise, radio frequency interference (RFI) and especially from the instrumentation noise that is due to necessary analog front-end $\mathrm{RF}$ amplification. The ratio between the effective collecting area of the $\mathrm{RF}$ receiving antennas and the system noise (after amplification and digitization) over the receiving bandwidth is a useful figure of merit for these systems, which explains why the collecting areas have become larger and the noise performance of the low noise amplifiers (LNAs) is critical. Signal to noise ratios (SNRs) well below $-60 \mathrm{~dB}$ over the operational bandwidth are typical. The RF cosmic photonic signals, as received by parabolic radio dishes (or co-planar antenna aperture arrays) on the surface of the earth either approximate temporally-stationary signals (e.g., signals from steadily-burning stars and their galaxies), or highly-dynamic broadband signals such as pulsars and quasars.

A potential application of real-time spatio-temporal 3-D FIR filters for the pre-processing of broadband digitized focal plane array (FPA) signals received by the dishes of the planned Square Kilometer Array (SKA) aperture synthesis radio telescope system has been proposed in [80]. The SKA will be the world's largest aperture synthesis radio telescope when it is completed in 2020 at an estimated cost of 2 billion [81]-[83]. Key science goals of the SKA include probing the dark ages, determination of the epoch of re-ionization, studies of the evolution of galaxies, dark energy, the origin and evolution of cosmic magnetism, search for the cradle of life and strong field tests of gravity to verify the general theory of relativity [81]-[83]. Some of these key science goals are to be achieved by analyzing broadband "transient" electromagnetic (EM) celestial signals of interest (SOIs), which are typically of less than $5 \mathrm{~s}$ duration and as short as $1 \mathrm{~ns}$ in the case of pulsars [82], [84].

Radio frequency focal plane arrays (FPAs) are under investigation as a means of increasing the field of view (FoV) of broadband aperture synthesis systems [85]. For the SKA, FPAs are under consideration for employment in the focal planes of many of the thousands of parabolic dishes [81]-[83] [86]. An FPA having a typical area of $3 \mathrm{~m}^{2}$ is capable of covering the so-called SKA lower mid-band (i.e., the frequency range 0.5 to $1.7 \mathrm{GHz}$ ) without significant diffraction losses [86]. It is expected that the multiple-beam capability of the FPA, combined with the large collecting area of each dish and the low noise figures of the low noise amplifiers (LNAs) over this frequency range will simultaneously facilitate both high sensitivity and high sky-survey speed for applications in aperture synthesis imaging [86], [87].

As illustrated in Fig. 7(a), far-field EM waves from celestial sources are reflected from the paraboloidal dish onto the focal plane where they are received by a rectangular array containing coplanar uniformlyspaced wideband elemental-antennas, such as Vivaldi antennas [88], [89] or patch antennas [90]. Currently, the number of elemental-antennas in an FPA is in the range of 100-200 [88]-[90]. The photonic response of each wideband elemental-antenna is converted to electronic form and amplified by corresponding elemental LNAs prior to analog pre-processing, A/D conversion and subsequent signal processing [90]. It is planned that the SKA will eventually consist of more than 3,000 paraboloidal dishes, implying over half a million elemental-antennas, LNAs, mixers and A/D converters in the complete system [81]-[83].

\section{B. Signal Received by FPAs and the Corresponding Spectra: A Review}

In the following, the properties of the ideal focal EM field of a dish-reflected celestial plane waves and its 3 -D space-time spectrum are derived using the Huygens' Principle Approximation [80] [91]. This focal field model has been verified using computer simulations [91]. Without loss of generality and for simplicity, in this approximation a regular-symmetrical paraboloidal-dish reflector where the focal-point is on the axis of the parabola has been assumed [see Fig. 7(a)]. Surface 


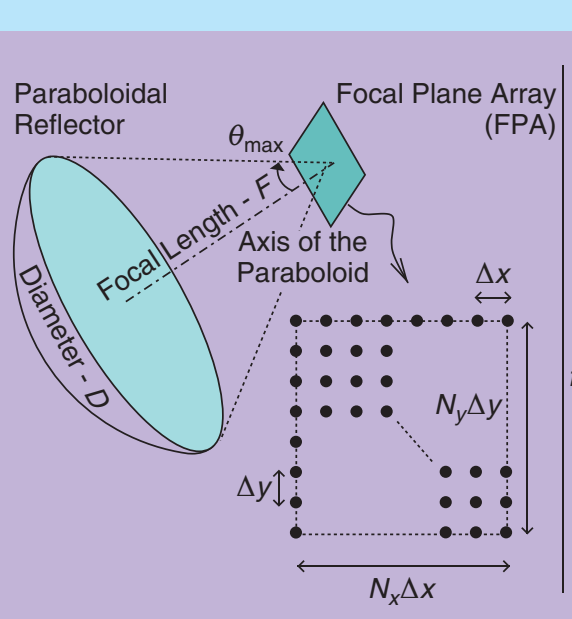

(a)

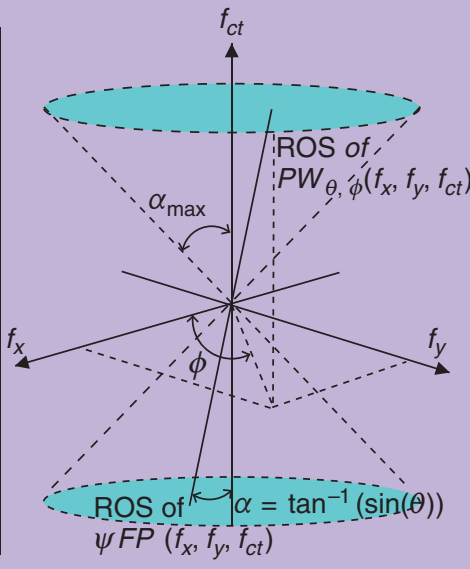

(b)

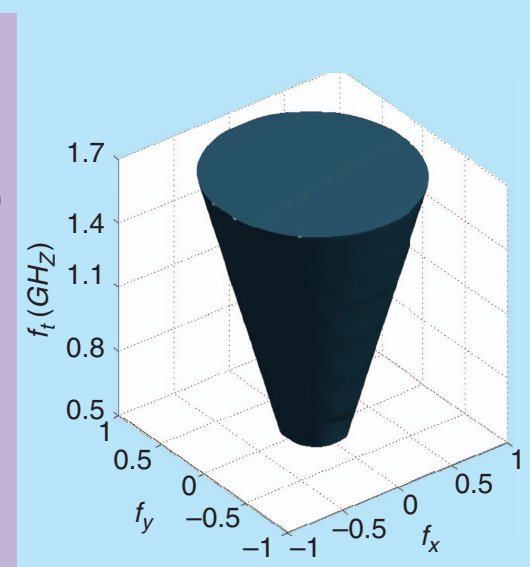

(c)

Figure 7. A paraboloidal reflector and a focal plane array (FPA) consist of $\left(N_{x}+1\right) \times\left(N_{y}+1\right)$ elements $(\mathrm{a})$. The ROS of the spectrum $P W_{\theta, \phi}\left(f_{x}, f_{y}, f_{c t}\right)$ of the plane wave and the predicted ROS of the spectrum of the focal plane field $\Psi F P\left(f_{x}, f_{y}, f_{c t}\right) ; \Psi=X, Y, Z(b)$. The equivalent iso-surface of the upper-side-band of the normalized spectrum of the co-polar component for frequencies $0.7-1.7$ $\mathrm{GHz}(\mathrm{c})$.

currents are induced on the reflecting surface of the dish in response to impinged EM signals and, according to the Huygens' Principle Approximation, these surface currents act as point sources that emit spherical wavefronts toward the focal region of the paraboloidal dish [92, ch. 15.]. Over a finite region of the focal plane, these spherical wavefronts can be closely approximated by infinitesimal spatio-temporal plane waves. Thus, the spatio-temporal EM field $\mathbf{f p}(x, y, c t)$, over a finite region around the focal-point on the focal plane, is formed by the superposition of such infinitesimal plane waves that radiate from all points on the reflector surface. Hence, the continuous-domain spatio-temporal 3-D EM field over the FPA may be written in the form of the spatial integration

$$
\mathbf{f p}(x, y, c t)=\sum_{\theta} \sum_{\phi} \mathbf{E}_{\theta, \phi}^{\mathrm{Pol}} p w_{\theta, \phi}(x, y, c t) .
$$

For these summed plane waves, the azimuth angle $\phi$ is in the range 0 to $2 \pi$, whereas the elevation angle $\theta$ is in the range $-\theta_{\max }$ to where $\theta_{\max }$ is given by dish geometry as

$$
\theta_{\max }=\tan ^{-1}\left(\frac{8(F / D)}{16(F / D)^{2}-1}\right) .
$$

The following 3-D spectral analysis is equivalently applied for all 3 components $f p_{k}(x, y, c t) ; k=x, y, z$ of the focal EM field $\mathbf{f p}(x, y, c t)$. According to section III the 3-D ROS of the 3-D spectrum $p w_{\theta, \phi}(x, y, c t)$ is given by the line of intersection of the two 3-D planes $f_{x}=\sin (\theta) \cos (\phi) f_{c t}$, and $f_{y}=\sin (\theta) \sin (\phi) f_{c t}$, both of which pass through the origin of the 3-D frequency space $\left(f_{x}, f_{y}, f_{c t}\right) \in \mathbb{R}^{3}$, as illustrated in Fig. 7(b). As $\phi$ varies from 0 to $2 \pi$, the ROS of $p w_{\theta, \phi}(x, y, c t)$ sweeps the surface of a cone having the half-cone-angle $\alpha=\tan ^{-1}(\sin (\theta))$. As both $\theta$ and $\phi$ are varied through their corresponding ranges, the trace of the ROS-line occupies the solid double-conic section having a half cone angle

$$
\alpha_{\max }=\tan ^{-1}\left(\sin \theta_{\max }\right),
$$

as shown in Fig. 7(b). This solid double-conic volume is the predicted ROSs of the spectra $\operatorname{XFP}\left(f_{x}, f_{y}, f_{c t}\right)$, $Y F P\left(f_{x}, f_{y}, f_{c t}\right)$ and $Z F P\left(f_{x}, f_{y}, f_{c t}\right)$ of the 3 components of the focal plane EM field $\mathbf{f p}(x, y, c t)$ of dish-reflected far-field signals [80]. For typical dimensions of the paraboloidal reflectors that have been proposed for the SKA [81], the half-cone angle $\alpha_{\max }$ varies in the range $35^{\circ}$ to $40^{\circ}$.

In FPAs, radio frequency interference (RFI) is mainly caused by terrestrial radio sources, such as spurious EM radiation from the electronic systems within the observation stations of the SKA itself, from broadcasting and wireless communication transmissions and from satellite broadcasting and communication transmissions. In the case of the RFI signals that are transmitted by satellites and received by an FPA, the ROS of the corresponding spectra is identical to the ROS of the spectra of celestial SOIs. Such interference is extremely difficult to suppress without attenuating the weak celestial SOIs that are in the angular vicinity of the interfering sources. However, the terrestrial RFI signals that are received directly on the FPA without being reflected 
from the paraboloidal-dish can be considered as spatiotemporal plane waves having typical inclination angles $\theta>75^{\circ}$ [93], [94]. Therefore, following Section II, the combinations of off-dish RFI signals $r f i(x, y, c t)$ can be expressed in the form

$$
r f i(x, y, c t)=\sum_{l} p w_{\theta, \phi}(x, y, c t)
$$

where $\theta \in\left[75^{\circ}, 90^{\circ}\right]$ and $\phi=[0,2 \pi]$. According to section II, the ROS of $\left(f_{x}, f_{y}, f_{c t}\right)$ is given by the lines of intersection between $f_{x}=\sin \left(\theta_{l}\right) \cos \left(\phi_{l}\right) f_{c t}$ and $f_{y}=\sin \left(\theta_{l}\right) \sin \left(\phi_{l}\right) f_{c t}$, for all $l$. For these lines of interaction, the inclination angles $\alpha_{l}$ are in the range of $\left[44^{\circ}, 45^{\circ}\right]$. Hence, inside the spectral region $\left\{\left|\omega_{x} ; \omega_{y}\right| \leqslant \pi \mid f_{c t} \in \mathbb{R}\right\}$, the ROS of the spectra of signals induced in the array of elementalantennas in response to off-dish RFIs, is located either on or close to the outer surface of the 3-D double-cone volume outlined in Fig. 7(b), where the half-cone angle $\alpha_{\max }=45^{\circ}$.

The thermal radiation from the distributed thermal sources around vicinity of the FPAs results another form of interference for the FPA receiver systems [95]. In [80], it is assumed that the distributed ground thermal sources is a collection of independent point-radiators and, on the FPA, the thermal radiation is approximated by a superposition of spatio temporal plane waves. However, the FPA is only receiving the thermal radiation plane waves for inclination angles $\theta>\theta_{\max }$ because for $\theta \leqslant \theta_{\max }$ the thermal radiation plane waves are blocked by the paraboloidal-dish [see Fig. 7(a)]. In [80], it has been shown that the ROS of the power spectral density (PSD) function of ground thermal noise is given by the volume between the two 3-D double-cones for which the half-cone angles are $\alpha_{\max }=\tan ^{-1}\left(\sin \theta_{\max }\right)$ and $\alpha_{\max }=45^{\circ}$, respectively.

The continuous-time signal from each LNA output is subjected to analog pre-processing prior to sampling: first, by amplification and bandpass filtering (in order to attenuate the out of band signals and to isolate the desired frequency band of interest (e.g., 0.5 to $1.7 \mathrm{GHz}$ ) [96]) and then by frequency down-shifting. For this analysis, direct frequency-down-conversion is assumed. The ROS of the spectrum of the pre-processed celestial SOIs is thereby converted to a $3-D$ frustum-shaped volume shown in Fig. 7(c) [80] [91]. Each down-converted LNA output is subjected to synchronous complex-quadrature sampling at the rate of $F_{\mathrm{s}}$. Ideally the sampling process transfers the volume $\mathbf{V}=\left\{\left|\omega_{x} ; \omega_{y}\right| \leqslant \pi \cup f_{c t} \in\left(f_{\mathrm{C}}-0.5 F_{S}, f_{\mathrm{C}}+0.5 F_{S}\right)\right\}$, which is centered at the point $\left(0,0, f_{\mathrm{C}}\right)$ in $\left\{\left|\omega_{x} ; \omega_{y}\right| \leqslant \pi \mid f_{c t} \in \mathbb{R}\right\}$, into the principle Nyquist cube; $\left\{\left|\omega_{x} ; \omega_{y} ; \omega_{t}\right| \leqslant \pi\right\}$, without distortion.

\section{Broadband Beamforming of FPA Space-Time Signals Using a Single 3-D FIR Frustum Filter}

In [80], the design of a complex-coefficient 3-D FIR frustum filter has been proposed. This filter is intended for the pre-processing of dish-reflected 3-D space-time digitized broadband FPA signals for the purpose of selectively recovering broadband pulsar signals to be subsequently used in pulsar-timing measurements and pulsar-profile studies. In subsection IV-B, it has been shown that the 3-D ROS of the spectrum of the digitized dish-reflected broadband celestial SOIs is a 3-D frustum whereas the 3 -D ROSs of the spectra of the interfering RFI signals and ground noise signals are located just outside that frustum. Furthermore, and most importantly, most of the digitized dominant 3-D broadband LNA noise spectrum also lies outside that frustum. Therefore, the proposed beamforming 3-D FIR filter is designed such that the frustum-shaped passband closely encompasses, and therefore transmits, most of the spectral components of the dish-reflected celestial broadband SOIs implying that the 3-D stopband covers the 3-D ROSs of the spectra of the RFI signals, of the ground noise signals and, to a large extent, of the 3-D LNA noise. Further, in order to achieve the maximum gain, the magnitude and the phase of the passband are selected such that the spectral components add coherently. It is recognized that fine refinements of the 3-D filter-coefficients may be necessary in order to optimize performance in a practical situation.

The 3-D spatio-temporal sampled-sequence from the FPA is $\tilde{x}(\mathbf{n}) ; \mathbf{n} \equiv\left(n_{x}, n_{y}, n_{t}\right)$, and the 3 -D non-separable unit impulse response of the beamforming 3-D FIR frustum filter is $\tilde{h}(\mathbf{n})$, implying that the beamformed 1-D output sequence $\tilde{y}\left(n_{t}\right)$, is given by the 3 -D discrete-domain convolution sum

$$
\begin{aligned}
& \tilde{y}\left(n_{t}\right)= \\
& \sum_{m_{x}=-L_{x}}^{L_{x}} \sum_{m_{y}=-L_{y}}^{L_{y}} \sum_{m_{t}=0}^{N_{t}} \tilde{x}\left(m_{x}, m_{y}, n_{t}-m_{t}\right) \tilde{h}\left(-m_{x},-m_{y}, m_{t}\right) .
\end{aligned}
$$

Note that $L_{i}=N_{i} / 2$; for $i=x, y$, and the FPA contains $\left(N_{x}+1\right) \times\left(N_{y}+1\right)$ elements so that the order of $\tilde{h}(\mathbf{n})$ is $\left[N_{x}, N_{y}, N_{t}\right]$.

In the case of inherently broadband pulsar-profile studies and pulsar-timing observations, a principal objective is to recover the pulse with minimal distortion of its shape. This obviously requires that the beamforming 3-D FIR frustum filter $\tilde{h}(\mathbf{n})$ be spatially-directed at the pulsar, which should be within the FoV of the FPA. In order to direct the beamformer towards a desired point within the FoV, the phase response, $\arg \left(H\left(\mathbf{e}^{\mathbf{j} \omega}\right)\right)$, of the transfer function $H\left(\mathbf{e}^{\mathrm{j} \omega}\right)$, must be linearly-shifted across the spatial-frequency space $\left\{\omega_{x} ; \omega_{y}\right\}$ [97] so that 


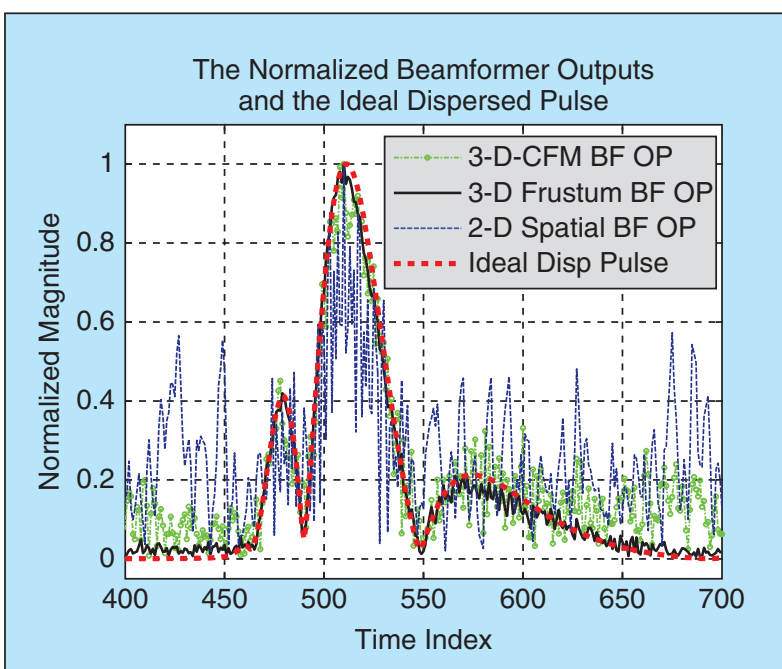

Figure 8. Examples of the normalized magnitudes of the recovered dispersed-pulses using the CFM beamformer (dot-dashed line, 3-D-CFM BF OP), the proposed 3-D FIR frustum filter-based beamformer for $\alpha_{\max }=30^{\circ}$ (solid line, 3-D Frustum BF OP), and the 2-D spatial-only beamformer (dashed line, 2-D Spatial BF OP) along with the magnitude of the ideal-dispersed pulse (dotted line, Ideal Disp Pulse).

the phase-center of the 3-D FIR filter coincides with the phase-center of the sampled focal FPA field signal $\widetilde{s i}(\mathbf{n})$ that resulted from the corresponding incident pulsar plane wave in the aperture of the dish. Further, in order to combine the spectral components of the SOI coherently, the phase-response of the beamforming filter should be selected such that

$$
\begin{aligned}
\angle H\left(\mathrm{e}^{\mathrm{j} \omega}\right) & \simeq-\angle S O I\left(\mathrm{e}^{\mathrm{j} \omega}\right) \\
\forall\left\{\omega_{x} ; \omega_{y} ; \omega_{t}\right\} & \in\left\{\left|\omega_{x} ; \omega_{y} ; \omega_{t}\right| \leqslant \pi\right\} .
\end{aligned}
$$

\section{A Comparative Study of the Recovery of Pulsar-Type Broadband Signals}

The performance of the proposed beamforming 3-D FIR frustum filter has been benchmarked against the conventional 2-D spatial-only beamformer and the 3-D conjugate-field-matching (CFM) beamformer that is based on a 3-D FIR filter structure of the same order as the frustum filter and is compared in the following. Given that pulsars are typically very weak radio sources, a group of (e.g., 100-1000) $15 \mathrm{~m}$ dish-receivers is needed in order to achieve the required SNR for pulsar-profile studies. Thus, for this example it is assumed that 1000 FPA-equipped dish-receivers of diameter (D) $15 \mathrm{~m}$ and focal-length (F) $6.75 \mathrm{~m}$ are directed at a distant emulated pulsar having an intrinsic twin-peak profile.

The bandwidth of operations for the FPAs is $0.5-1.7 \mathrm{GHz}$. Each FPA contains $289=(17 \times 17)$ elemental antennas arranged in a square-gird with the inter-element distance of $8.817 \mathrm{~cm}$. The photonic responses of the FPAs are contaminated with receiver noise, ground noise and off-dish RFI spanning the bandwidth $[0.74,1.7] \mathrm{GHz}$. For this simulation, the noisy and RFI contaminated test sequences $\widetilde{x_{k}}(\mathbf{n}) ; k=1, \ldots, 1000$ are synthesized using the focal field synthesis program FFS [91] and Fourier techniques [1] [97]. Each of the 1000 test sequences $\widetilde{x_{k}}(\mathbf{n}) ; k=1, \ldots, 1000$ of $\operatorname{size}\left(17 \times 17 \times 2^{10}\right)$ contains a dispersed-pulse contaminated with noise and RFI, which is sampled at the rate of $1.2 \mathrm{GHz}$. Note, each of the 289 time records of $\widetilde{x_{k}}(\mathbf{n})$ spans a duration of approximately 0.853 microseconds. For each $\widetilde{x_{k}}(\mathbf{n})$, the signal to receiver noise ratio (SRNR) was chosen as $-10 \mathrm{~dB}$, the signal to ground noise ratio (SGNR) as $-20 \mathrm{~dB}$ and the signal to interference ratio (SIR) as $-30 \mathrm{~dB}$.

For the purpose of comparison, each $\widetilde{x_{k}}(\mathbf{n})$ is processed by the proposed 3-D FIR frustum filter-based beamformer, which corresponds to a cone having half-cone angle $\alpha_{\max }$, of order [16,16,20], the 2-D spatial only beamformer of order $[16,16]$ and the 3 -D CFM beamformer of order $[16,16,20]$. The three groups of beamformed outputs are time aligned, averaged and normalized. The resulting three time sequences corresponding to the three beamforming methods, contain the normalized recovered dispersed-pulses. Fig. 8 shows the examples of such normalized dispersedpulses recovered using the proposed 3-D FIR frustum filter-based beamformer (solid) where $\alpha_{\max }=30^{\circ}$, using the 3-D CFM beamformer (dot-dashed) and using 2-D spatial only beamformer (dashed). The ideal-dispersed pulse (dotted), is also shown in Fig. 8. According to that the proposed 3-D FIR frustum filter-based beamformer achieves lower distortion of the recovered broadband signals compared to the 3-D CFM beamformer and 2-D spatial-only beamformer.

\section{2-D/3-D IIR Filter Circuits for RF Antenna Arrays}

In this section we first discuss the 2-D spatio-temporal filters for processing signals from linear sensor arrays (see Fig. 3) and then extend the discussion to 3-D spatio-temporal filters for processing signals from planar sensor arrays. Discrete-time domain realization of 2-D IIR filters can be achieved by converting 2-D continuous-domain Laplace prototype transfer function of the form $H_{a}\left(s_{x}, s_{c t}\right)$ into the corresponding 2-D z-transform domain. This is achieved at guaranteed practicalBIBO stability [41] by employing the 2-D BLT given by $s_{\alpha}=\left(1-z_{\alpha}^{-1}\right) /\left(1+z_{\alpha}^{-1}\right) \alpha \in\{x, c t\}$. The application of the 2-D BLT leads to the transfer function $H_{D}\left(z_{x}, z_{c t}\right)$, which in turn can be used for obtaining 2-D difference equations, by following the inverse $\mathbf{z}$-transform under ZICs. The difference equations are iterative and recursive over 
both discrete space and time dimensions, and can be computed on a real-time basis using digital arithmetic circuits or software algorithms. The above-mentioned straightforward method for obtaining a set of MD difference-equations starting from the continuous-domain Laplace functions leads to direct-form SFGs, where a spatial delay of $N_{x}$ units corresponds to the signal emanating from the neighboring spatial node $N_{x}$ units away from the current grid position of the difference equation, and a temporal delay of $N_{t}$ time units corresponds to a sampled delay of $N_{t}$ clock cycles, with respect to the temporal sampling clock of the filter. Analog realizations can be achieved by discretizing only the spatial Laplace variable.

\section{A. Raster Scan and Systolic Array Digital Architectures}

Two types of architectures have been proposed for the realization of 2-D IIR spatio-temporal filters. The raster scanned architectures employ a high-speed ADC which samples the sensor array in a time multiplexed scheme. An example in 3-D processing would be the processing of digital video sequences pixel by pixel. However, we focus here on systolic array realizations of MD filters that achieve massive throughputs up to RF rates at the cost of circuit complexity and power consumption [98], [99], unlike raster scanned architectures which are slower, smaller, and lower in power consumption. In a systolic array MD filter implementation, there exists a dedicated analog front end for each sensor, which is connected to a dedicated high-speed ADC (Fig. 3). Each ADC feeds a single processor which we call a parallel processing core module (PPCM). Each PPCM is a parallel digital arithmetic circuit that computes one spatio-temporal output sample of the underlying 2-D difference-equation in a single clock cycle, once all internal pipelines have been filled with data samples. The PPCMs themselves form a tightly interconnected mesh of processors which directly implement the iterative computations needed for the recursive MD difference equations that describe the filtering operations over discrete space and time.

The scanned array architectures deliver a real-time throughput of one-sample-per-clock-cycle (OSPCC), whereas the massively parallel systolic array architectures have a throughput of one-frame-per-clock-cycle (OFPCC). That is, given $N_{x}$ sensors, the systolic array version is $N_{x}$ fold greater in both throughput and computational complexity compared to the raster scanned architecture. Systolic arrays are more suitable for throughput intensive applications such as radar signal processing or radio astronomy where the sensors are $\mathrm{RF}$ antennas having several $\mathrm{GHz}$ of bandwidth. The realtime throughput of systolic array architectures can be increased by employing multi-rate signal processing, leading to a throughput of multiple-frames-per-clockcycle (MFPCC) [56], [100].

\section{B. Frequency Planar Space-Time Beam Plane Wave Filters}

An Nth-order 2-D IIR frequency planar beam space time plane wave filter transfer function takes the form [101]

$$
H_{D}\left(z_{x}, z_{c t}\right)=\frac{\left(1+z_{x}\right)^{N}\left(1+z_{c t}\right)^{N}}{1+\sum_{i=0}^{N} \sum_{j=0}^{N} b_{i j} z_{x}^{-i} z_{c t}^{-j}}
$$

where $b_{00}=0$ and $N=1,2,3 \ldots$, is used for a high-speed RF beamforming application. Applications for electronically scanned RF beams in receiver mode exist in wireless cellular communications, RF tracking and source location, cognitive radio, radar and microwave imaging, RF habitat monitoring and electronic signal intelligence [101]. A general $N$ th order 2-D passive prototype network shown in Fig. 9 is used to obtain the $N$ th order IIR transfer function in (21), by following the 2-D BLT. Table 1 lists the closed-form expressions of the feedback filter coefficients $b_{i j}$ for the filter order $N=1,2,3$ of (21) [101]. The direct form realization is the simplest to design in systolic array hardware. However, it is not the most efficient in terms of computational complexity.

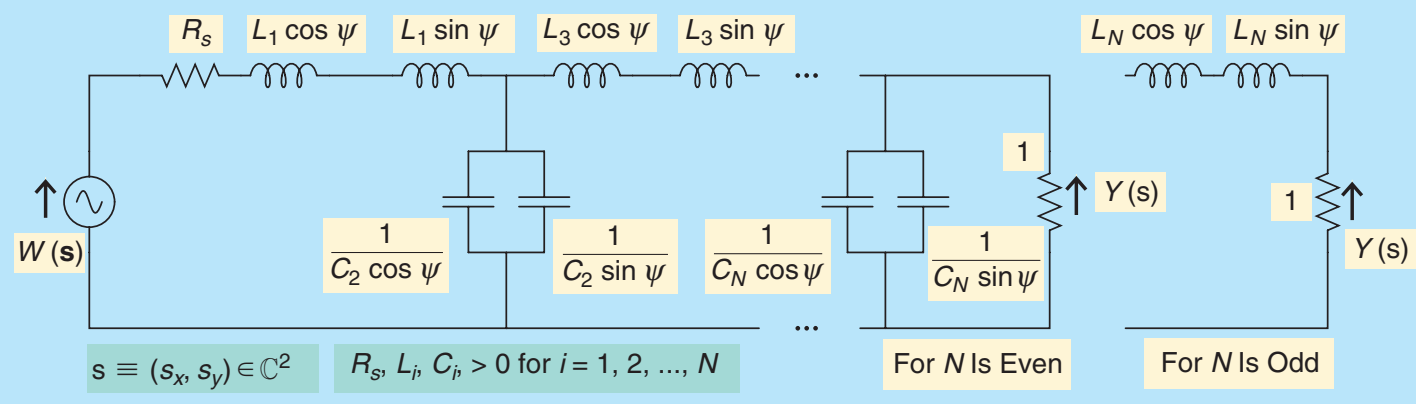

Figure 9. The Nth order 2-D Laplace domain passive prototype network. 
Table 1.

Filter design equations showing algebraically defined filter coefficients as functions of LC-ladder prototype network parameters and steered angle $\tan \psi=\sin \theta$. Here, - refers to dot product between vectors.

\begin{tabular}{|c|c|c|}
\hline $\begin{array}{l}\text { Order } \\
\text { of Filter }\end{array}$ & Arbitrary Coefficients $e_{m}$ & Coefficients $b_{i j}$ \\
\hline 1 & $\begin{array}{l}L_{i}=L \cos \psi \\
L_{j}=L \sin \psi \\
e_{1}=1+R_{s} \\
e_{2}=L_{i}, e_{3}=L_{j}\end{array}$ & $\begin{array}{l}b_{i j}=\hat{e} . \hat{\beta}_{i j}, b_{00}=0 \\
\hat{\mathrm{e}}=\left(e_{1}, e_{2}, e_{3}\right) / \epsilon \\
\epsilon=e_{1}+e_{2}+e_{3} \\
\beta_{01}=(1,0,-1) \\
\beta_{10}=(1,-1,0) \\
\beta_{11}=(1,-1,-1)\end{array}$ \\
\hline 2 & $\begin{array}{l}L_{i}=L_{1} \cos \psi, \\
L_{j}=L_{1} \sin \psi, \\
C_{i}=C_{2} \cos \psi, \\
C_{j}=C_{2} \sin \psi \\
e_{1}=1+R_{s} \\
e_{2}=L_{i}+R_{s} C_{i}, \\
e_{3}=L_{j}+R_{s} C_{j}, \\
e_{4}=L_{i} C_{i}, e_{5}=L_{j} C_{j}, \\
e_{6}=L_{i} C_{j}+L_{j} C_{i} .\end{array}$ & $\begin{array}{l}b_{i j}=\hat{\mathrm{e}} . \hat{\beta}_{i j}, b_{00}=0 \\
\hat{\mathrm{e}}=\left(e_{1}, e_{2}, e_{3}, e_{4}, e_{5}, e_{6}\right) / \epsilon \\
\epsilon=e_{1}+e_{2}+e_{3}+e_{4}+e_{4}+e_{6} \\
\beta_{01}=(2,2,0,2,-2,0) \\
\beta_{02}=(1,1,-1,1,1,-1) \\
\beta_{10}=(2,0,2,-2,2,0) \\
\beta_{11}=(4,0,0,-4,-4,0) \\
\beta_{12}=(2,0,-2,-2,2,0) \\
\beta_{20}=(1,-1,1,1,1,-1) \\
\beta_{21}=(2,-2,0,2,-2,0) \\
\beta_{22}=(1,-1,-1,1,1,1)\end{array}$ \\
\hline 3 & $\begin{array}{l}L_{i}=L_{1} \cos \psi, \\
L_{j}=L_{1} \sin \psi, \\
C_{i}=C_{2} \cos \psi, \\
C_{j}=C_{2} \sin \psi, \\
L_{k}=L_{3} \cos \psi, \\
L_{i}=L_{3} \sin \psi, \\
e_{1}=1+R_{s} \\
e_{2}=L_{i}+L_{k}+R_{s} C_{i}, \\
e_{3}=L_{j}+L_{i}+R_{s} C_{j} \\
e_{4}=L_{i} C_{i}+L_{k} C_{i} \\
e_{5}=L_{j} C_{j}+L_{i} C_{j} \\
e_{6}=L_{i} C_{j}+L_{j} C_{i}+L_{k} C_{j}+L_{i} C_{i}, \\
e_{7}=L_{i} L_{k} C_{i}, e_{8}=L_{j} L_{i} C_{j} \\
e_{9}=L_{i} L_{k} C_{j}+L_{i} L_{i} C_{i}+L_{j} L_{k} C_{i}, \\
e_{10}=L_{i} C_{i} L_{i}+L_{j} L_{k} C_{j}+C_{i} L_{j} L_{i}\end{array}$ & $\begin{array}{l}b_{i j}=\hat{e} . \hat{\beta}_{i j}, b_{00}=0, \\
\hat{\mathrm{e}}=\left(e_{1}, e_{2}, e_{3}, e_{4}, e_{5}, e_{6}, e_{7}, e_{8}, e_{9}, e_{10}\right) / \epsilon, \\
\epsilon=e_{1}+e_{2}+e_{3}+e_{4}+e_{5}+e_{6}+e_{7}+e_{8}+e_{9}+e_{10} \\
\beta_{01}=(3,3,1,3,-1,1,3,-1,1,-1) \\
\beta_{02}=(3,3,-1,3,-1,-1,3,3,-1,-1) \\
\beta_{03}=(1,1,-1,1,1,-1,1,-1,-1,1) \\
\beta_{10}=(3,1,3,-1,3,1,-3,3,-1,1) \\
\beta_{11}=(9,3,3,-3,-3,1,-9,-9,-1,-1) \\
\beta_{12}=(9,3,-3,-3,-3,-1,-9,9,1,-1) \\
\beta_{13}=(3,1,-3,-1,3,-1,-3,-3,1,1) \\
\beta_{20}=(3,-1,3,-1,3,-1,3,3,-1,-1) \\
\beta_{21}=(9,-3,3,-3,-3,-1,9,-9,-1,1) \\
\beta_{22}=(9,-3,-3,-3,-3,1,9,9,1,1) \\
\beta_{23}=(3,-1,-3,-1,3,1,3,-3,1,-1) \\
\beta_{30}=(1,-1,1,1,1,-1,-1,1,1,-1) \\
\beta_{31}=(3,-3,1,3,-1,-1,-3,-3,1,1) \\
\beta_{32}=(3,-3,-1,3,-1,1,-3,3,-1,1) \\
\beta_{33}=(1,-1,-1,1,1,1,-1,-1,-1,-1)\end{array}$ \\
\hline
\end{tabular}

By using the method of negative resistance based predistortion [102], we can effectively change the SFG of the filter such that the number of multipliers needed in a systolic realization is minimized. Negative resistance based elemental pre-distortion is achieved by inserting small negative resistances in series with the inductive branch impedances of the LC ladder prototype, and compensating for this action by placing positive resistances of equal magnitude also in series with the negative resistances, thereby avoiding any change to the driving point impedance of the filter. Thereafter, by judicious selection of each negative resistance, one can obtain a prototype network which results in minimum number of multipliers in the equivalent difference equation realization. Fig. 10 shows both direct form (on left) as well as differential form (on right) SFGs and their corresponding digital hardware realizations on systolic arrays for a first $\operatorname{order}(N=1) 2$-D IIR beam plane wave filter.

\section{Interference Rejection}

\section{for Wireless Communication}

Consider a scenario where the 2-D IIR beam filters are used in an ultra-wideband communication system in a typical office environment. A model of a typical office was created in a computational electromagnetic simulator, where the dielectric properties of walls and partition 


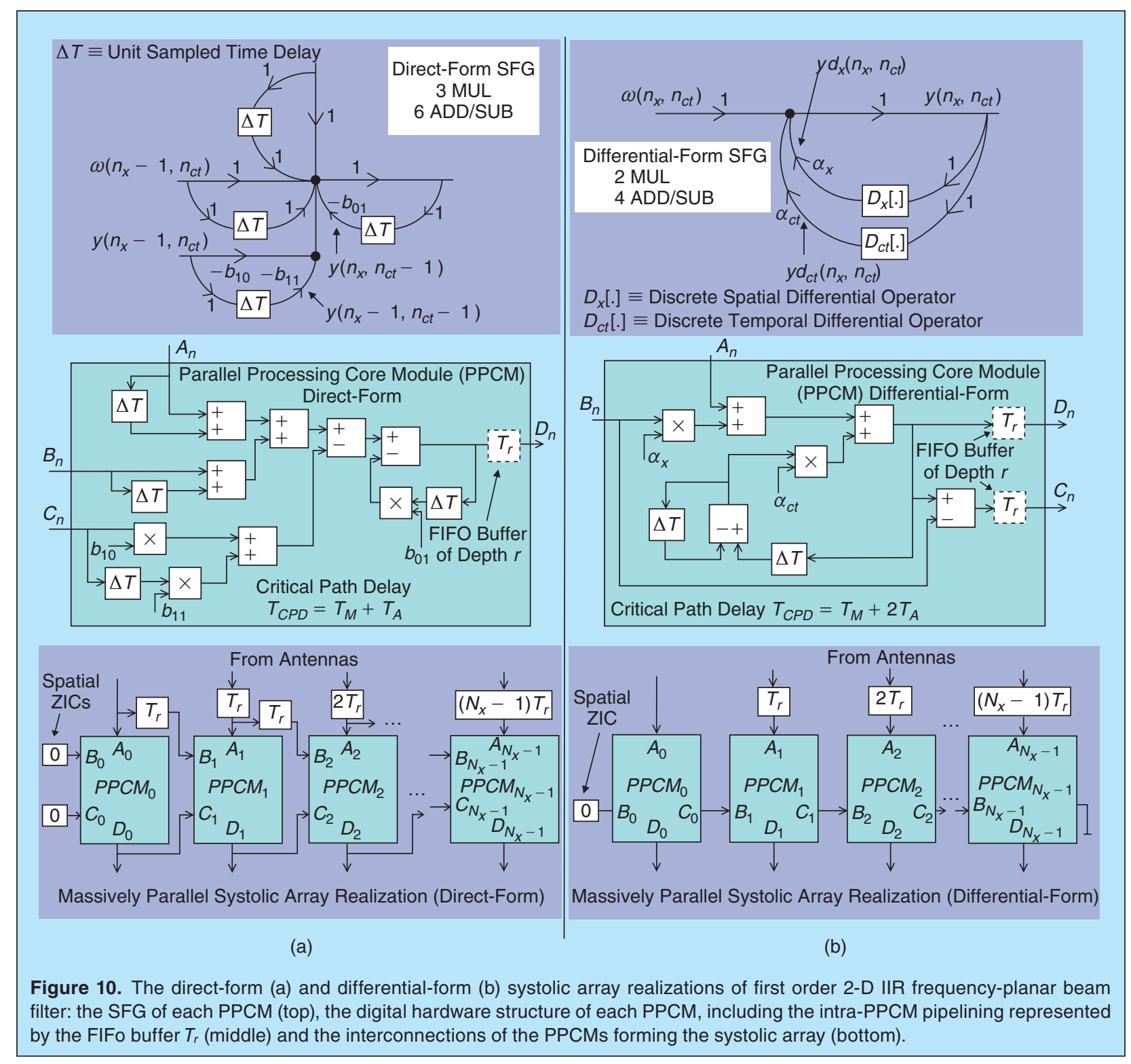

material were accurately accounted for in a map of the wireless radio propagation channel. Bipolar phase shift keying (BPSK) modulation using Hermite pulses was assumed for the signalling scheme, which used a bandwidth of about $3 \mathrm{GHz}$ at a symbol rate of about 400 symbols/second. A physics based radio channel model was created using finite difference time domain (FDTD) modeling of Maxwell's equation and solved iteratively using a GPU based FDTD accelerator. Monte Carlo simulations were carried out assuming 2-D IIR beam filtering using first-order beam plane wave filtering. Several arrays of antennas were considered, starting from dense arrays sampled at the spatial Nyquist distance, followed by sparse arrays having varying degrees of sparsity. This investigation showed promising results in terms of Monte Carlo simulated bit error rate (BER) versus signal to interference and noise (SINR) curves (assuming 3-bit quantization of the signal at each antenna). The performance of the 2-D IIR space time beam plane wave filter was also investigated when quantization and rounding errors due to fixed point digital arithmetic within the systolic array processors were taken into account. A reconfigurable FPGA systolic array processor was tightly coupled to the FDTD radio propagation model and a multitude of simulations were carried out in Monte Carlo style, for obtaining the relevant statistically significant data that quantified the role of fixed point errors on BER vs. SINR in a typical ultra wideband wireless communication scenario, where the proposed filters were used for combating massive amounts of interference/jamming [67], [103]. It was established that 3-bit precision at the ADCs were more than sufficient 


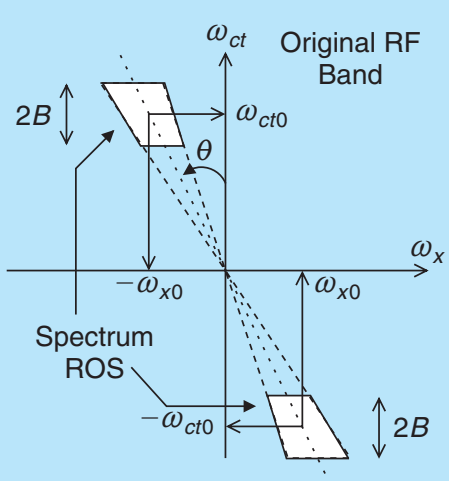

(a)

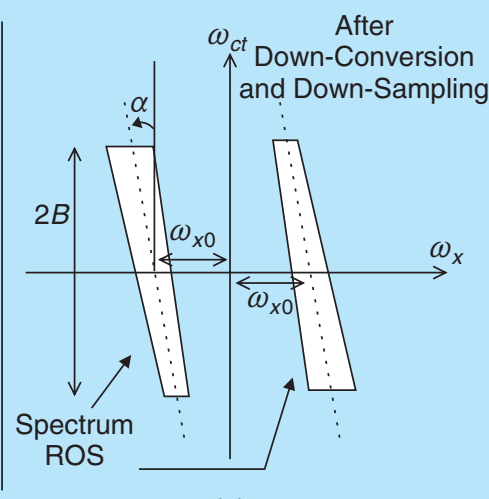

(b)

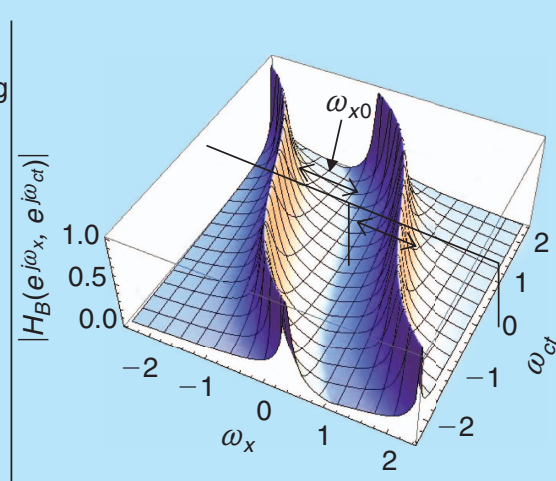

(c)

Figure 11. The ROS of the 2-D spatio-temporal spectrum of a partially broadband plane wave signal in RF domain (a) and in IF domain after down-conversion and down-sampling (b). The magnitude frequency response of a spatially bandpass 2 -D IIR beam filter (c).

for achieving excellent performance from the system. Complete details of the findings can be obtained by reading [67], [103]. In connected work conducted at the University of Toronto, the above-mentioned office environment was created in an electromagnetic test environment. The antenna array consisted of a sparse array of linear configuration with ultra wideband BAVA elements. The experiment validated the FDTD simulations using real-life measured data from the experimental setup [104].

\section{Beamformers Having Down}

\section{Converters in the RF Front-End}

Up to this point, our discussion has been limited to the case of ultra wideband beamforming using 2-D IIR frequency planar beam space time plane wave filter technology. However, in many practical situations, the signal of interest is a modulated carrier wave having bandwidths up to $25 \%$. To accommodate such signals, we can modify the frequency planar beam filter to take into account spectral properties of down converted array signals. As shown in Fig. 11(a) the ROS of the 2-D spectrum of a partially broadband carrier modulated $\mathrm{RF}$ signal is changed as shown in Fig. 11(b) when downconversion and down-sampling is employed. The 2-D spectrum ROS is no longer passing through the origin and has a spatial frequency shift denoted by $\omega_{x 0}$. For the selective enhancement/jamming of such partially broadband down-converted IF signals, a 2-D IIR filter having spatially modulated passband is required. An example of a first order spatially bandpass 2-D IIR beam filter magnitude frequency response is shown in Fig. 11(c). The spatially-bandpass 2-D IIR beam filter transfer function $H_{B}\left(z_{x}, z_{c t}\right)$ can be derived based on the regular 2-D IIR transfer function $H_{D}\left(z_{x}, z_{c t}\right)$ given in (21), by following an impulse response modulation technique proposed in [105], [106]. The modified expressions for the filter coefficients by following the impulse response modulation can be found in [105], [106]. This spatially-bandpass beam plane wave filter was simulated for Gaussian modulated plane waves having sinusoidal carrier frequencies. An $N=64$ element array was assumed. The desired signal was at a carrier frequency of $f_{C}=1 \mathrm{GHz}$, with DOA $\psi_{0}=30^{\circ}$. The single-sided bandwidth was $B=250 \mathrm{MHz}$. The sampling frequency was $F_{S}=2\left(f_{C}+B\right)=2.5 \mathrm{GHz}$. The fractional bandwidth is therefore $2 B / f_{C}=0.5$ (50\%). The interference rejection capabilities of the spatially-bandpass beam filter can be observed from Fig. 12 [106].

Two interference waves identical to the desired signal except for the spatial DOAs $\psi_{1}=10^{\circ}$ and $\psi_{2}=60^{\circ}$ was considered for the verification of interference rejection. A down-sampling factor of $N_{S}=5$ is used in this temporally bandpass example where mixers are assumed at each antenna, which reduces the required clock frequency to $F_{C L K}=F_{S} / N_{S}=500 \mathrm{MHz}(=2 B)$. The 2-D input spectrum following down conversion, filtering and down-sampling steps is shown in Fig. 12(a), where both desired and undesired signal spectra are present. The 2-D magnitude frequency response of the resulting output signal is shown in Fig. 12(b). Clearly, the plane wave filter shows the directional enhancement of the desired signal having DOA $\psi_{0}=30^{\circ}$, while suppressing wideband interference/jamming arriving at other DOAs. The computational complexity of the 2-D spatiallybandpass beam plane wave filter is significantly smaller compared to traditional algorithms based on FFT-based phased array beamforming [106]. Five prototypes of 


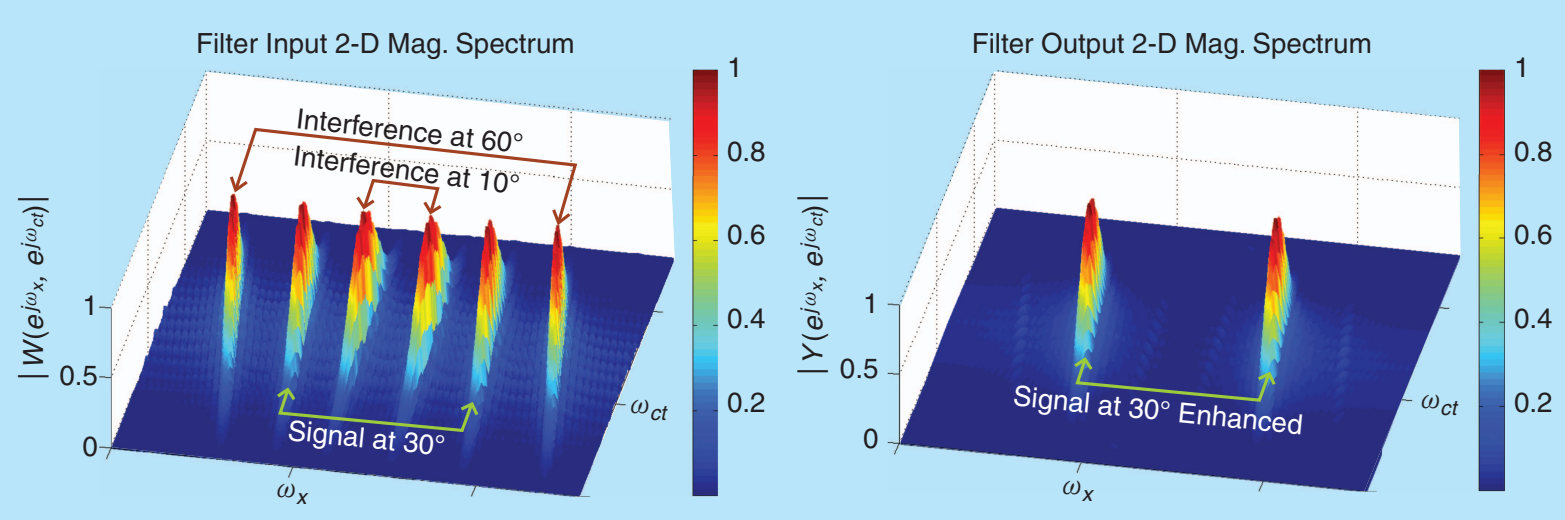

Figure 12. The 2-D magnitude spectrum of the input signal to the SBP 2-D IIR beam filter containing one desired signal and two interference (a). The spectrum of the filtered output, where the desired spectra at the spatial DOA $30^{\circ}$ is enhanced (b).

this spatially bandpass filter were tested on a $65 \mathrm{~nm}$ CMOS asynchronous FPGA platform [106].

\section{E. Getting RF Rate Throughput \\ from Available Technology}

A critical aspect of real-time RF beamforming using MD digital signal processing is computational throughput. Ultra wideband RF signals can have several $\mathrm{GHz}$ of bandwidth, which lead to multi-GHz sampling frequencies at the ADCs. When there are many antennas in the array, the total computational throughput can be very significant. The demanding throughput levels usually rule out the use of software realizations including GPU realizations with custom designed systolic array processors that maximize computational throughput using multilevel massive parallelism being the only viable option. Furthermore, multi-GHz ADC data streams necessitate very high clock rates on the systolic array. Available digital CMOS technologies level off in clock speeds at about $4 \mathrm{GHz}$, requiring polyphase signal processing methods for $\mathrm{RF}$ antenna signal processing to achieve bandwidths of more than $2 \mathrm{GHz}$ [56], [100]

This is common practice for the case of FIR filterbanks used in radio astronomy, for example. Our recent research has extended polyphase filter bank hardware to the case of 2-D IIR fan filter banks using novel multirate SFG manipulations that combine polyphase clocking with the concept of time-interleaved ADCs to achieve real time frame sample rates for 2-D IIR beam filtering at several times the maximum clock frequency of the implementation technology [56], [100]. The methods have been applied to both direct form as well as lowcomplexity differential form SFGs leading to polyphase realizations on FPGA for both types of architectures [56], [100]. An example of a multi-rate PPCM architecture is shown in Fig. 13(a), where two non overlapping phases are used to obtain a real time throughput of 2FPCCs. In the SFG shown in Fig. 13(a), the input signal transform $W\left(n_{x}, z_{c t}\right)$ is converted to corresponding multi-rate components $W_{j}\left(n_{x}, z_{c t}\right)$ for $j=1,2$ by employing down-sampling and delay blocks. However, if time interleaved ADC is employed in the antenna array, these sampling rate conversion blocks can be absorbed into the time interleaved sampling happening inside the ADC. Fig. 13(b) shows the resulting systolic array configuration that uses time interleaved ADC with 2 non overlapping clocks $\phi_{1}$ and $\phi_{2}$. Details of SFGs and design equations of this multi-rate systolic array implementations, which offer the capacity to operate at real-time throughputs greater than what is offered by current technology as scaled by Moore's Law, can be found in [56], [100].

\section{F. Systolic Arrays for 3-D IIR Space-Time Digital Filters}

We consider the 3-D IIR plane wave filtering case where the incident plane wave signal is sampled using a uniformly spaced rectangular array of antennas. Such rectangular arrays are known as dense aperture arrays and are highly sought after in deep space imaging systems such as the Square Kilometer Array (SKA) radio telescope, which can in fact be a good example application following sufficient future research. The conventional method for obtaining a steerable beam is the use of well-known aperture synthesis algorithms based on phased arrays. In a conventional phased array system, the sampled signals from each antenna is processed using a combination of polyphase FIR filter banks and FFT processors for channelization, followed by a phased summation achieved by an array of complex multipliers which accurately tune the phase of each signal such that coherency is achieved for plane waves arriving at a desired DOA. 


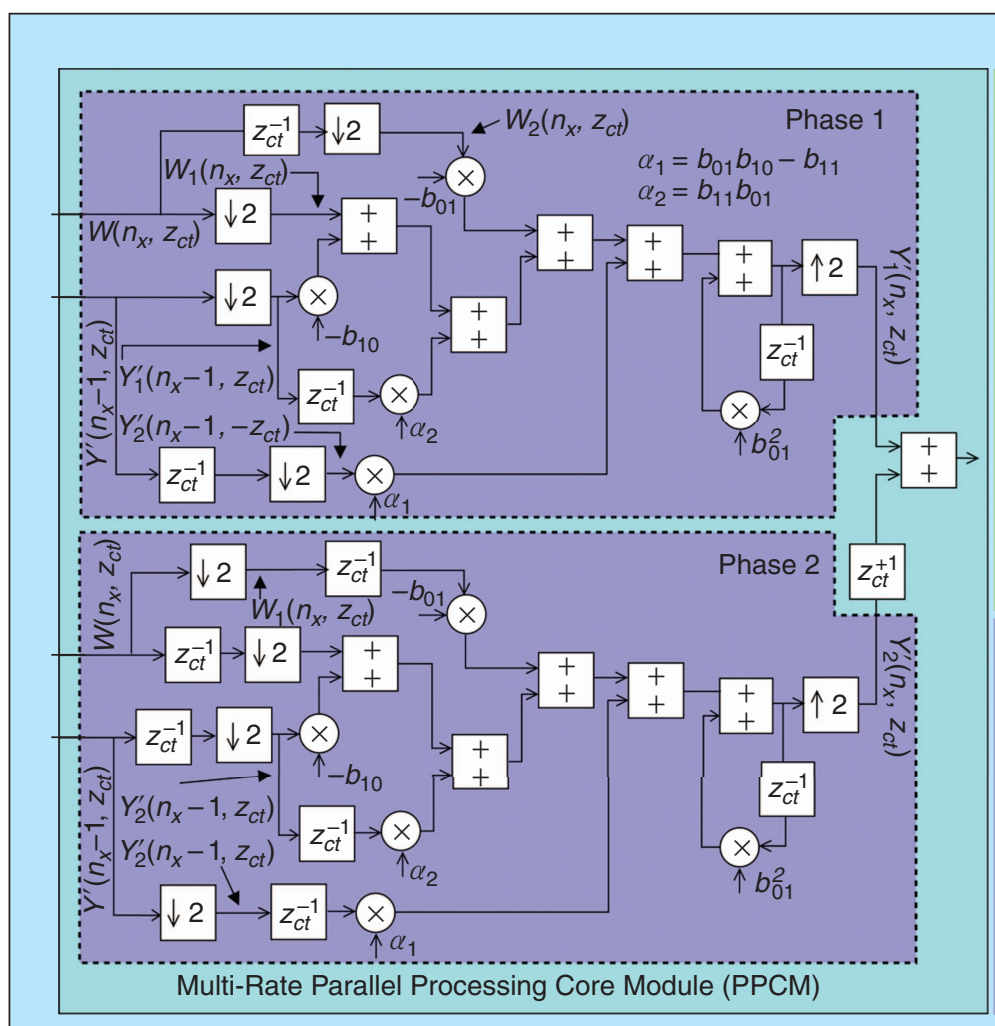

(a)

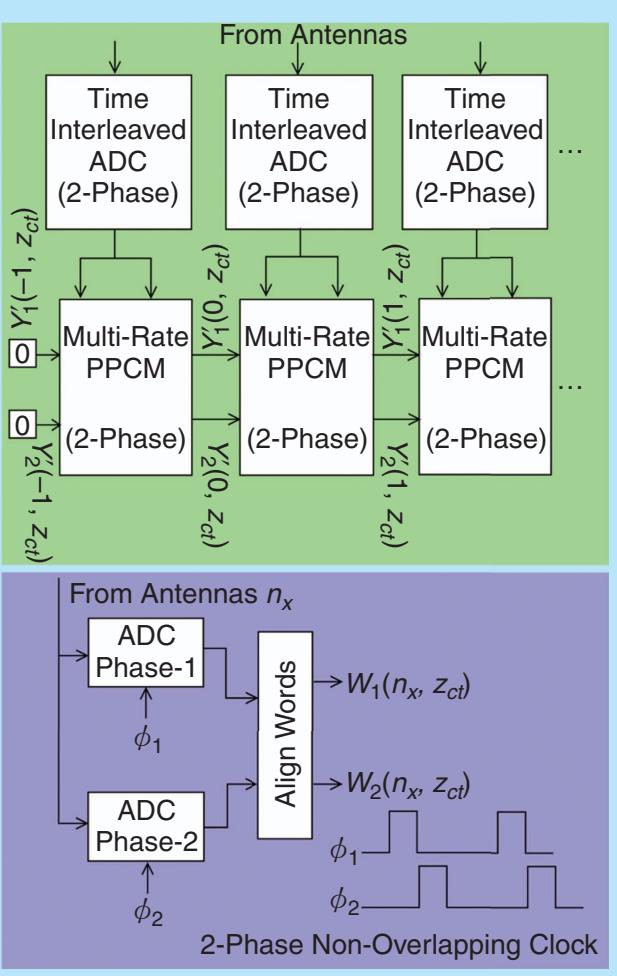

(b)

Figure 13. Multi-rate PPCM architecture example that delivers 2 frames-per-clock-cycle throughput (a). The multi-rate systolic array architecture that employs time interleaved ADC with two non-overlapping clocks (b).

Unlike phased arrays, the 3-D IIR cone filter approach [69], [10] achieved precision ultra wideband steerable beams exploiting the well known geometrical properties of the 3-D spectrum of plane waves. By realizing a digital filter having cone shaped passbands, which are aligned along the DOA of the desired plane wave signal, the 3-D filtering with highly selective enhancement of propagating plane waves based on their DOA is achieved in the presence of high interference and noise. The computational complexity (number of multipliers) of the 3-D cone filter banks are $90 \%$ smaller than the multiplier complexity of FFT-based digital phased array beamformers [10], when compared in terms of the mean square error of the magnitude frequency response.

Fig. 14(a) shows the formation of a 3-D beam shaped passband by two intersecting frequency planar passbands. Such 3-D IIR beam filters can then be used in a filter bank configuration having multiple bands, where each band is realized using a frequency beam filter followed by a temporal bandpass filter. Fig. 14(b) shows a 3-D IIR filter bank having multiple cone shaped passbands. The cone-shaped magnitude frequency response for the single passband case is shown in Fig. 14(c). Such multiple passband 3-D IIR cone filters provide frequency independent far field array patterns having multiple $\mathrm{RF}$ beams. A mathematically computed example array pattern with $4 \mathrm{RF}$ beams is shown in Fig. 14(d).

\section{G. Wave Digital Filters (WDFs)}

Usually, input output transfer functions of a filter relate the $\mathbf{z}$-transform of an input voltage or current sequence to the z-transform of an output voltage or current sequences. These sequences are typically available as sampled space time discrete sequences in the MD case. However, WDFs operate on the principle of discrete scattering (S) parameters, where the difference equations relate transfer functions of a two-port network defined in terms of forward and backward traveling wave quantities. WDFs were first proposed by Fettweis [107] in 1970s for the original application involving audio signal processing for wireline telecommunications, and have since been extended to the MD case for other applications such as image and video processing, for example [108]. A raster scan hardware SFG of a 2-D WDF can be described using conventional WDF parameters, as shown in Table 2 for the case of first-order multi-beam plane wave filters [60], [108]. 


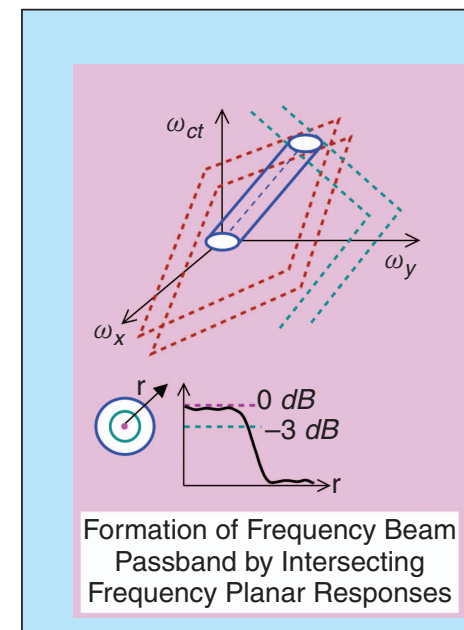

(a)

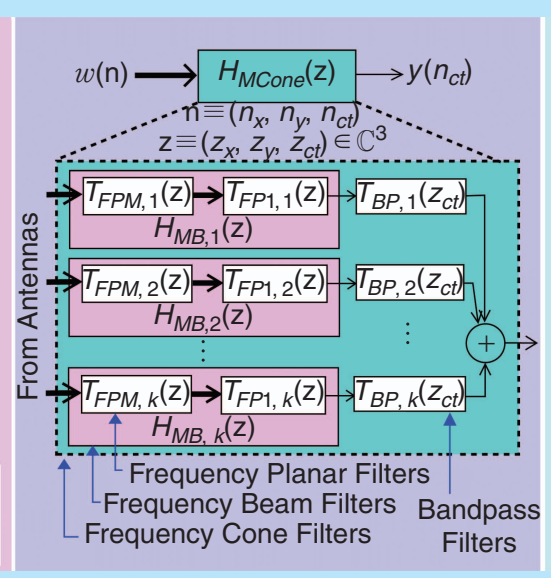

(b)

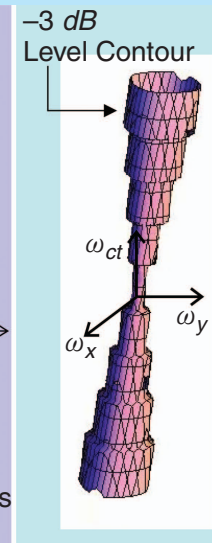

(c)
Far Field Beam Pattern of 3-D IIR Cone Filter Bank with 4 Passbands

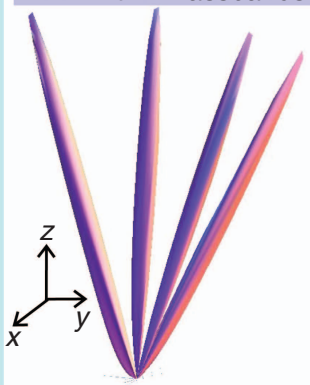

(d)

Figure 14. Formation of a 3-D beam shaped passband by intersecting frequency-planar passbands (a). Structure of a 3-D IIR filter having multiple cone shaped passbands in the 3-D spatio-temporal frequency domain (b). The $-3 \mathrm{~dB}$ level contour of the magnitude frequency response of 3-D IIR single passband cone filter (c). Far field array pattern (3-D polar response) of a 3-D IIR cone filter having four passbands, leading to four RF beams (d).

The WDFs require wave adapters to be realized using digital hardware VLSI circuits [60]. For the case of first-order 2-D plane wave filters, the corresponding WDF adapters and coefficients are shown in Fig. 15 and Table 2 for 1,2 and 3 beams [60].

\section{H. Analog 2-D Beam Plane Wave Filters}

Sensor signals are continuous-time before being sampled and digitized by ADCs. If MD filters can be made to operate in continuous-time mode, one can take advantage of the resulting analog circuits because

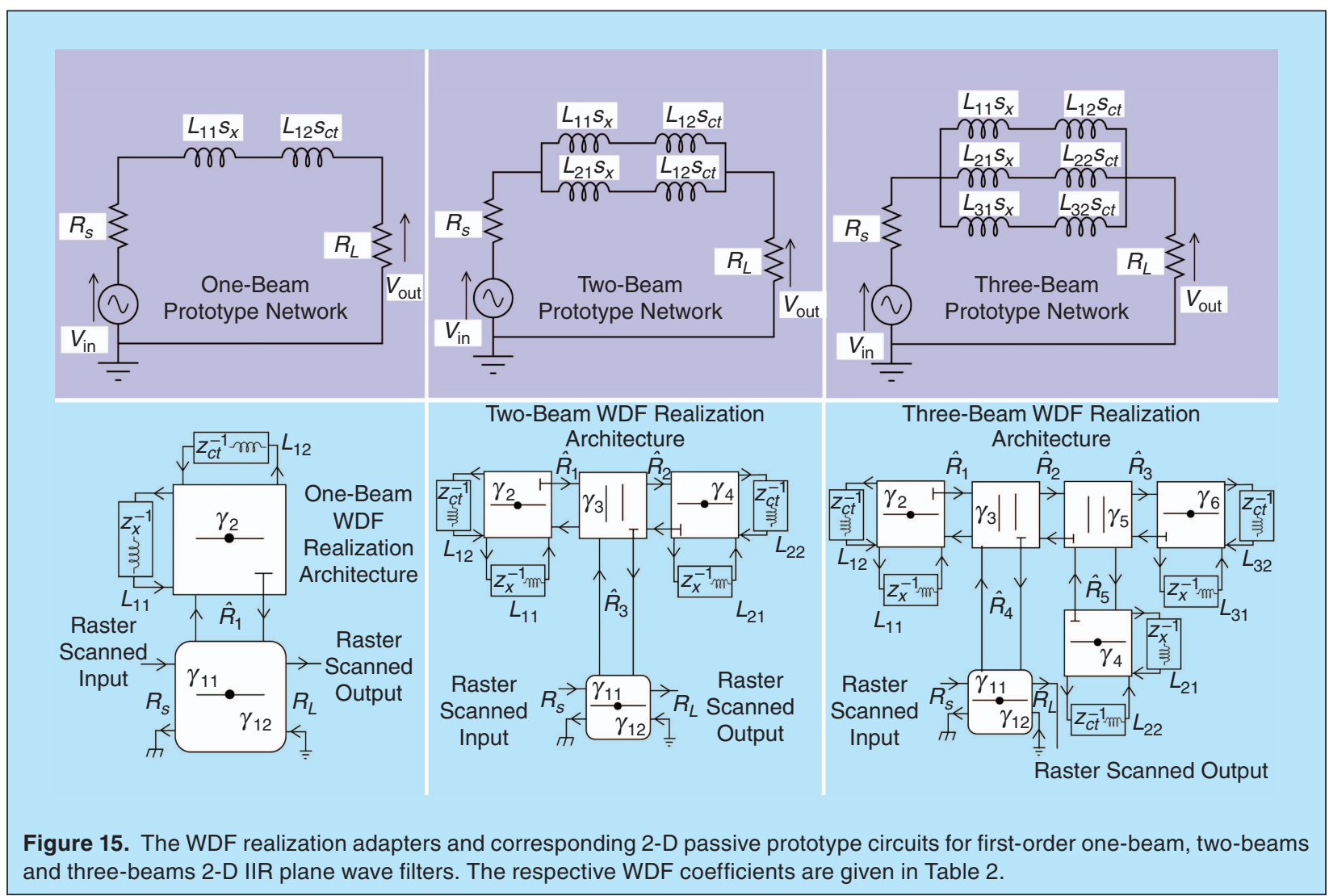


TABLE 2.

WDF design equations for 1st order multi-beam classical LC ladder networks.

\begin{tabular}{|llccc|}
\hline Type of Filter & & \multicolumn{3}{c|}{ WDF Design Equations } \\
\hline $\begin{array}{l}\text { 1-beam filter from 1st } \\
\text { order 1-D prototype }\end{array}$ & $\hat{R}_{1}=L_{12}+L_{11}$ & $\gamma_{2}=\frac{L_{12}}{L_{12}+L_{11}}$ & $\gamma_{11}=\frac{2 R_{s}}{R_{s}+\hat{R}_{1}+R_{L}}$ & $\gamma_{12}=\frac{2 R_{L}}{R_{s}+\hat{R}_{1}+R_{L}}$ \\
\hline $\begin{array}{l}\text { 2-beam filter from 1st } \\
\text { order 1-D prototype }\end{array}$ & $\hat{R}_{1}=L_{12}+L_{11}$ & $\hat{R}_{2}=L_{22}+L_{21}$ & $\gamma_{2}=\frac{L_{12}}{L_{12}+L_{11}}$ & $\gamma_{3}=\frac{\left(1 / \hat{R}_{1}\right)}{\left(1 / \hat{R}_{1}\right)+\left(1 / \hat{R}_{2}\right)}$ \\
& $\hat{R}_{3}=\frac{\hat{R}_{1} \hat{R}_{2}}{\hat{R}_{1}+\hat{R}_{2}}$ & $\gamma_{4}=\frac{L_{22}}{L_{22}+L_{21}}$ & $\gamma_{11}=\frac{2 R_{s}}{R_{s}+\hat{R}_{3}+R_{L}}$ & $\gamma_{12}=\frac{2 R_{L}}{R_{s}+\hat{R}_{3}+R_{L}}$ \\
\hline $\begin{array}{l}\text { 3-beam filter from 1st } \\
\text { order 1-D prototype }\end{array}$ & $\hat{R}_{4}=\frac{\hat{R}_{1} \hat{R}_{2}}{\hat{R}_{1}+\hat{R}_{2}}$ & $\gamma_{2}=\frac{L_{12}}{L_{11}+L_{12}}$ & $\gamma_{3}=\frac{\left(1 / \hat{R}_{1}\right)}{\left(1 / \hat{R}_{1}\right)+\left(1 / \hat{R}_{2}\right)}$ & $\gamma_{5}=\frac{\left(1 / \hat{R}_{3}\right)}{\left(1 / \hat{R}_{3}\right)+\left(1 / \hat{R}_{5}\right)}$ \\
& $\gamma_{6}=\frac{L_{32}}{L_{32}+L_{31}}$ & $\gamma_{4}=\frac{L_{22}}{L_{22}+L_{21}}$ & $\gamma_{11}=\frac{2 R_{s}}{R_{s}+\hat{R}_{4}+R_{L}}$ & $\gamma_{12}=\frac{2 R_{L}}{R_{s}+\hat{R}_{4}+R_{L}}$ \\
\hline
\end{tabular}

such realizations do not require sampling in the time domain. Because analog filters are not sampled, they are free of aliasing in the time frequency domain and do not require clock distribution networks. Analog filters process signals without quantizing them, making such filters free of quantization noise and limit cycle oscillations. However, analog filters do have their own problems. For example, they are more prone to analog noise injection, harmonic and intermodulation distortion, and EM interference issues. Mixed-domain filters are described by recursive difference equations along spatial dimensions, and by differential equations along time dimension. Practical circuit realizations consist of a parallel interconnection of multiple-input multipleoutput analog circuits, which are interconnected in such a way that the iterative spatial difference equations required for IIR filters in multiple space-time dimensions is obtained.

For example, in an analog 2-D IIR beam filter, the prototype has a 2-D Laplace domain transfer function $H_{a}\left(s_{x}, s_{c t}\right)=(R) /\left(R+L_{x} s_{x}+L_{c t} s_{c t}\right)=\left(Y_{a}\left(s_{x}, s_{c t}\right)\right) /\left(W_{a}\left(s_{x}, s_{c t}\right)\right)$. We apply the bilinear transform to the spatial Laplace variable $s_{x}$ while leaving the temporal Laplace variable $s_{c t}$ unchanged, thereby leading to the mixed-domain transfer function $H_{m}\left(z_{x}, s_{c t}\right)=(R) /\left(R+L_{x}(2 / \Delta x) \cdot\left(z_{x}-1 / z_{x}+1\right)+L_{c t} s_{c t}\right)$ $=\left(Y_{m}\left(z_{x}, s_{c t}\right)\right) /\left(W_{m}\left(z_{x}, s_{c t}\right)\right)$. The mixed-domain realization shows reduced bilinear warping [33]. This formulation leads to an array of two-dimensional analog modules (2DAMs) that implement the difference-differential equations in an interconnection of analog circuits. We are currently working on realizing mixed-domain
2-D/3-D IIR filters at up to $10 \mathrm{GHz}$ of bandwidth for $\mathrm{RF}$ array processing.

\section{4-D Light Field Filters for Imaging in Challenging Environments}

In 1966 artificial intelligence pioneer Marvin Minsky famously assigned "computer vision" as an undergraduate summer project. Although computer vision has made massive strides in the nearly 50 years since, the field remains young in that many "simple" problems remain unsolved, and significant advances lie ahead. Much of the recent progress in computer vision has exploited the recent feasibility of using very large datasets. The related problems of place, object and text recognition, for example, are being successfully tackled in Google's image-based search by providing millions of exemplar images to what is essentially an appearance-based clustering algorithm [109]. To recognize human gestures such as kicking, driving and dancing, Microsoft's Kinect software was trained using on the order of a million 3-D images [110].

As progress is made in these high-level tasks, so too are advances being made at the lowest levels of computer vision. One might have assumed that camera technology would be well established this far into the development of computer vision, yet in the past few years three important new technologies for measuring 3-D images have emerged: time of flight cameras, which employ the finite propagation rate of light to measure depth; structured light cameras such as the Kinect, which employ known projected patterns of light; and plenoptic (aka light field) cameras, which generalize 
stereo cameras by measuring images on a regular spatial grid.

Considerable effort is going into developing effective ways to use the images measured by each of these new camera technologies, with significant recent advances in pose estimation, scene modelling and gesture recognition, amongst others [110]-[113]. Most of these advances are data heavy and processor intensive, relying on iterative optimization schemes and sophisticated feature extraction. At the same time, the images measured by each of these cameras can be approached using the same multi-dimensional signal processing (MDSP) techniques discussed earlier in this article. These methods are linear, robust, have constant and predictable runtime, and offer competitive or even superior performance to competing methods at a fraction of the complexity. Our focus in this section is specifically on light field imagery, for which methods from MDSP are a particularly good match.

\section{A. Light Field Cameras}

A conventional camera measures the light rays passing through a single aperture. Referring to Fig. 16(a), this is like looking at the world through a peephole, yielding two-dimensional (2-D) images $w\left(n_{u}, n_{v}\right)$, where $n_{u}$ and $n_{v}$ represent the angle of arrival of incoming rays. Stereo and multiple-camera approaches to computer vision generally treat their inputs as arrays of independent 2-D angular images. Light field imaging differs in that it samples over two additional, spatial dimensions. This is like measuring through a regularly spaced grid of peepholes, as depicted Fig. 16(b).

We depict the 4-D light field signal $w\left(n_{x}, n_{y}, n_{u}, n_{v}\right)$, where each sample of $w$ encodes the value of a single ray in the scene, with a specific position and direction. A convenient way to map indices to rays is via the two-plane parameterization depicted in Fig. 16(c). The indices $n_{x}$ and $n_{y}$ encode the spatial coordinates of a ray's intersection with an $x, y$ reference plane coincident with the aperture array, and the angular indices $n_{u}$ and $n_{v}$ encode the ray's point of intersection with a second reference plane $u, v$, at arbitrary distance $D$.

There are several practical ways to measure a light field, perhaps the most obvious of which is to build an array of conventional cameras, as in the Stanford array [114] depicted in Fig. 17(a). Recently more compact cameras have become available, based on the use of lenselet arrays mounted in the optical path of otherwise conventional cameras. These include the "original" plenoptic camera as described by $\mathrm{Ng}$ et al. [115], and the "focused" plenoptic camera described by Lumsdaine and Georgiev [116]. The two models differ in their spatial/angular resolution trade offs. Examples of commercially available versions of each are shown

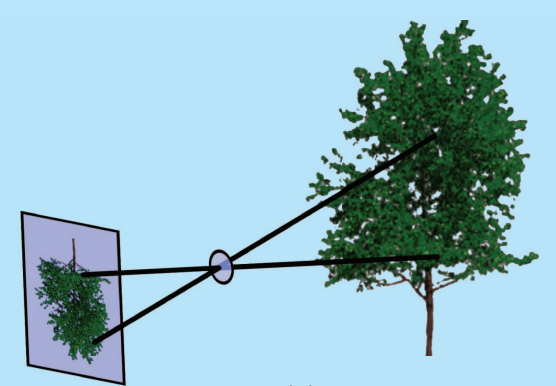

(a)

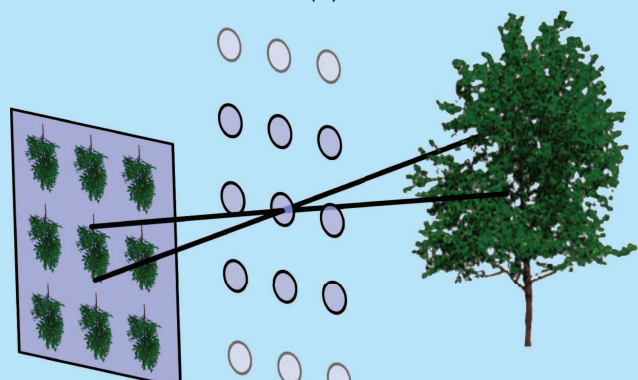

(b)

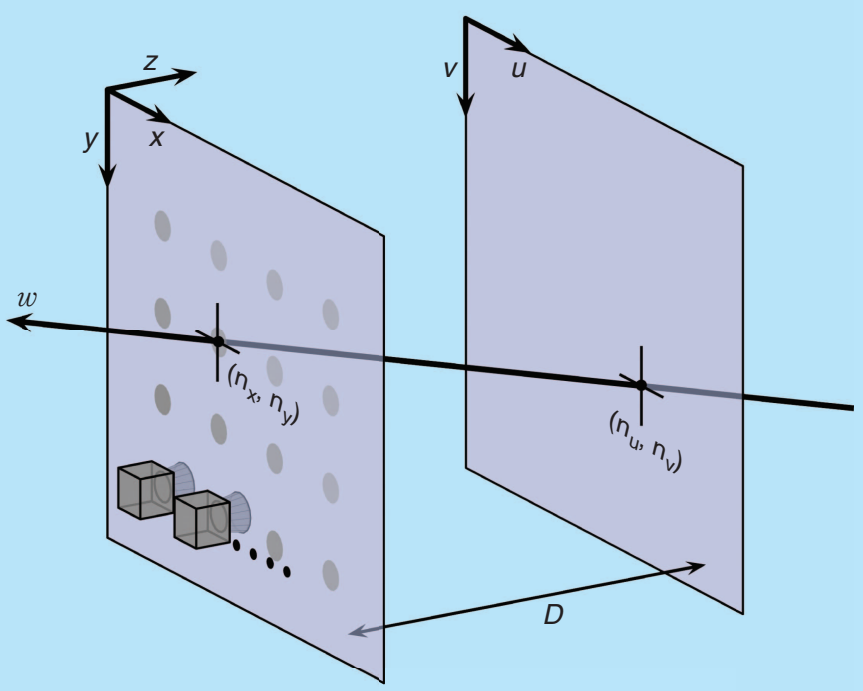

(c)

Figure 16. Whereas a conventional camera measures light passing through a single aperture (a), a light field camera samples on a regular spatial grid (b), measuring light in terms of both position and direction; the two-plane parameterization (c) is a convenient way to map each light field sample to a single ray in space. 


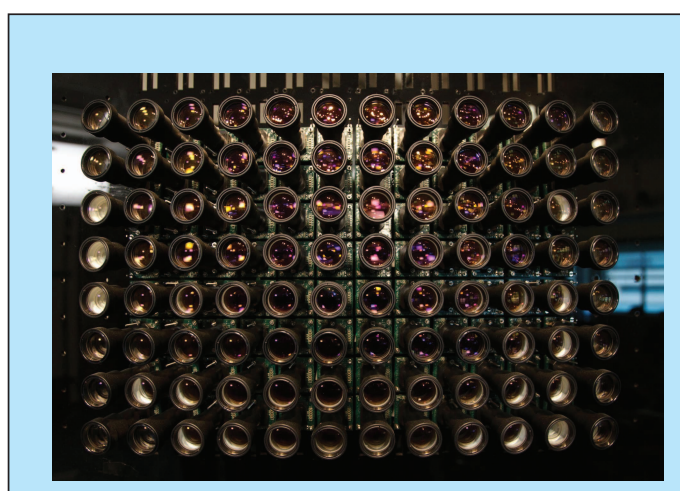

(a)

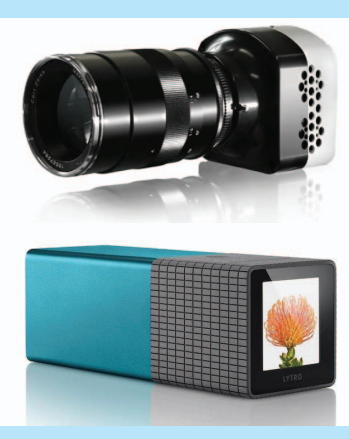

(b)

Figure 17. Three models of light field camera: (a) an array of conventional cameras, and (b) two variants of lenselet-based camera, with different spatial/angular resolution trade offs. (Images are by Stanford University, Raytrix $\mathrm{GmbH}$, and Lytro Inc.)

\section{B. Light Field Properties}

One of the key insights in light field processing is that both the textural and geometric information associated with a scene are encoded in the regularly sampled 4-D signal $w$. This means that a wide range of filtering techniques traditionally reserved for texture can now also be applied to geometry. In this section we briefly describe some of the important characteristics of light field signals, which we later exploit to accomplish conventionally complex tasks using simple filters.

Visualizing the light field can be accomplished in 2-D slices, for example allowing $n_{u}$ and $n_{v}$ to

in Fig. 17(b), and example sensor images showing differing ratios of angular and spatial samples are shown in Fig. 18. A final model of light field camera makes use of attenuating masks to modulate incoming light, allowing the light field to be retrieved through a demodulation process [117], [118]. Each camera model has its own decode process, mapping raw sensor pixels to 4-D light field coordinates. Though each model features different light gathering and resolution trade-offs, they all measure a 4-D function representing the light traveling through a scene in terms of position and direction. The filters we describe below therefore apply to light fields measured by any of these camera models, following at most a resampling process, or adjustment of the filter's parameters. vary while holding $n_{x}$ and $n_{y}$ fixed at a specific spatial location. An example of such a visualization is shown in Fig. 19(a), with $n_{x}$ and $n_{y}$ fixed near the central spatial sample. The scene depicts a beer mat being held in place by a wooden dowel in front of a poster of a supernova. The next frame in the figure visualizes the same light field in $n_{u}$ and $n_{x}$, near the central sample in $n_{v}$ and $n_{y}$. This depicts a fundamental relationship in the angular and spatial behavior of light: points in 3-D space appear as straight lines in angular/spatial slices. These straight lines are a manifestation of parallax motion: each scene element has apparent motion along the angular dimension $u$ as a function of the spatial dimension $x$. The differing slopes of these lines are due to the differing depths of their corresponding scene elements.

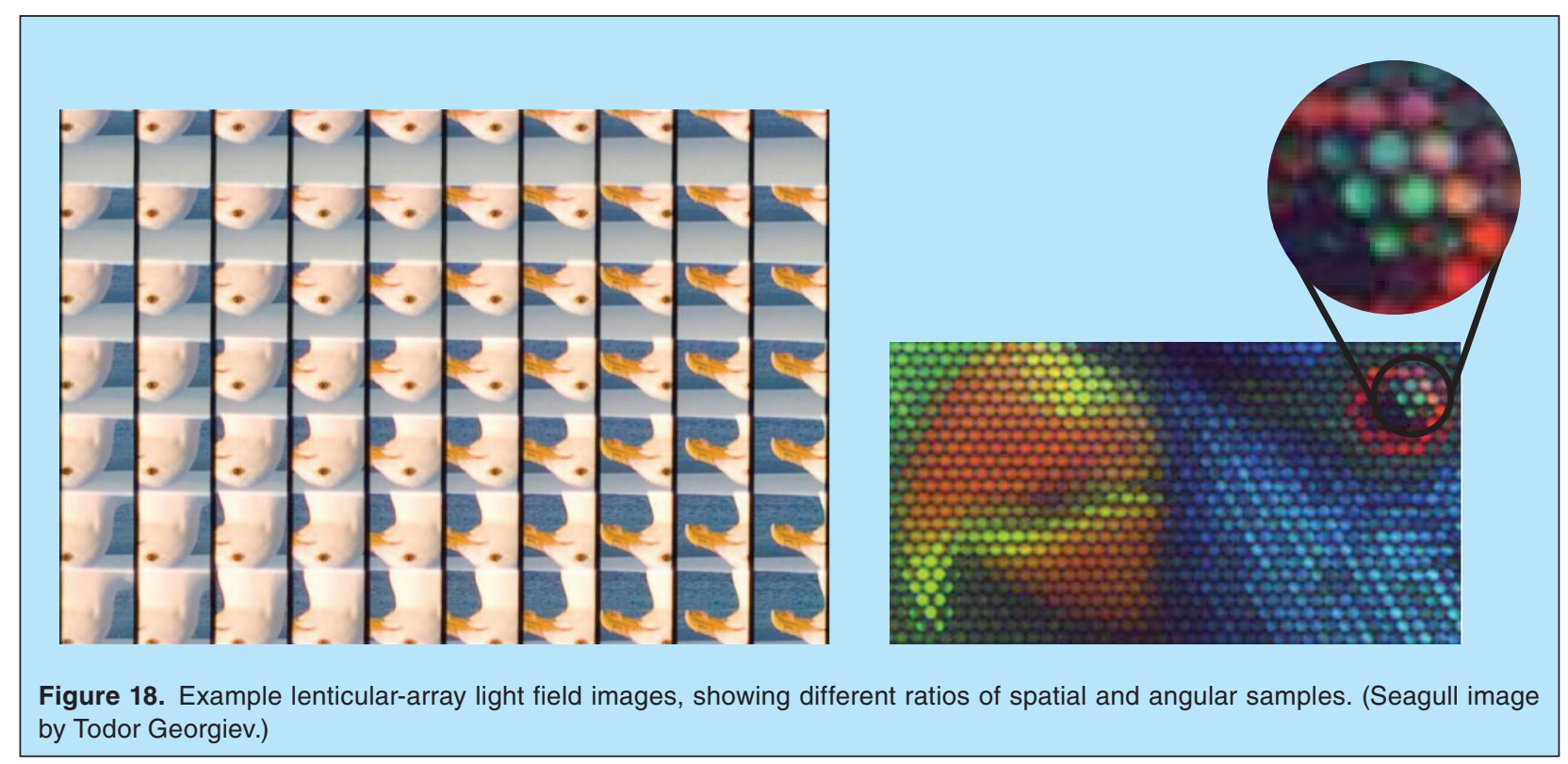




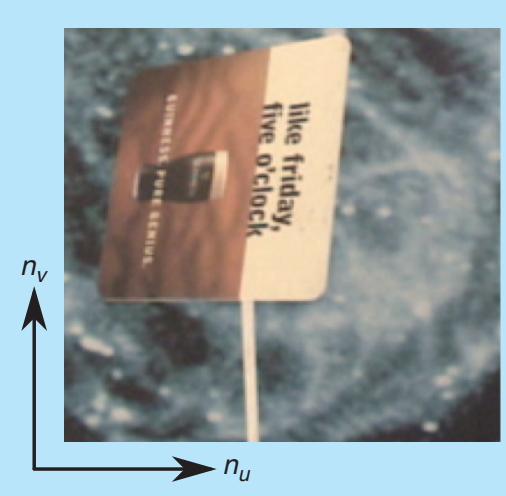

(a)

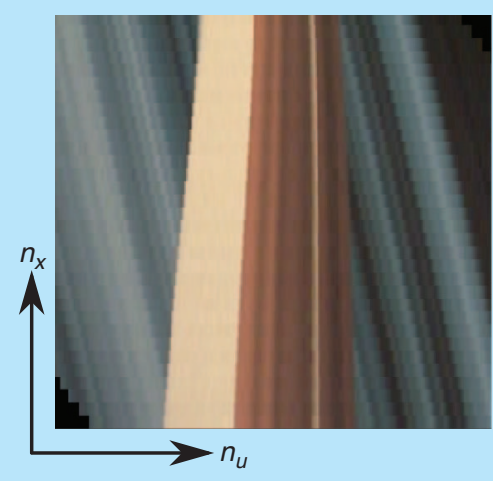

(b)

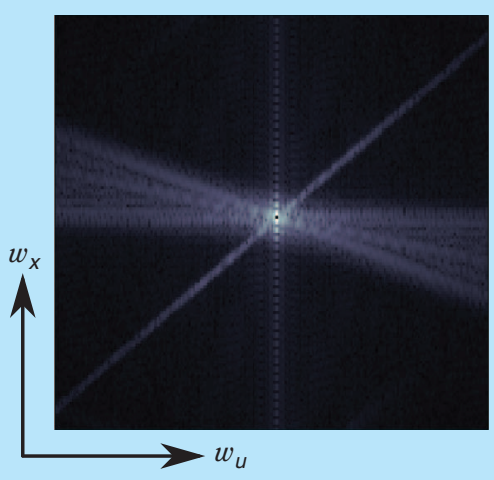

(c)

Figure 19. The left pane (a) depicts a light field as viewed from a single spatial location; the same light field viewed as a horizontal slice through the beer mat (b) shows how parallax motion appears in the light field as straight lines in the $n_{u}, n_{x}$ dimensions; a consequence of the resulting straight lines (planes in 4-D) is that the frequency-domain ROS of the light field (b) is also a set of planes; slopes of both spatial and frequency-domain planes depend on the depth of the corresponding scene element.

The same observations hold in $n_{v}$ and $n_{y}$ slices, and taken together these observations describe parallax motion as a correspondence between a point $P$ in 3-D space, and a plane in the 4-D light field [68], [119]. In the continuous spatial domain, the relationship can be written as

$$
\left[\begin{array}{l}
u \\
v
\end{array}\right]=\left(\frac{D}{P Z}\right)\left[\begin{array}{l}
P_{X}-x \\
P_{Y}-y
\end{array}\right]
$$

Though we do not derive it here, it follows from the point-plane correspondence (22) that the frequencydomain light field will also exist as a set of planes [68]. This observation is remarkably similar to those made around spatio-temporal plane waves, with the key differences that here all dimensions are spatial, and the correspondence between spatial- and frequencydomain planes is in 4-D. Fig. 19(c) depicts a slice, in the frequency-domain indices $\omega_{u}$ and $\omega_{x}$, of the discrete Fourier transform (DFT) of the beer mat light field. Note the range of plane orientations forming a roughly horizontal fan shape: this is the frequency-domain region of support (ROS) of the beer mat, which occupies a range of depths in the scene. The diagonal plane seen crossing from the bottom-left to the top-right corresponds to the poster in the background, which lies at a single depth.

More formally, the frequency-domain planar ROS of a point $P$ can be described as

$$
\omega_{x} / \omega_{u}=\omega_{y} / \omega_{v}=D / P_{z}-1
$$

Note the plane's orientation depends only on the depth of its corresponding point, $P_{z}$, and not its other spatial coordinates. For a scene which exists over a range of scene depths,

$$
Z_{\text {MIN }}<P_{Z}<Z_{\text {Max }}
$$

we can reorganize the frequency-domain relationship (23) into three simultaneous constraints

$$
\begin{aligned}
m_{\mathrm{MIN}} & <\omega_{x} / \omega_{u}<m_{\mathrm{MAx}}, \\
m_{\mathrm{MIN}} & <\omega_{y} / \omega_{v}<m_{\mathrm{MAx}}, \\
\omega_{x} / \omega_{u} & =\omega_{y} / \omega_{v},
\end{aligned}
$$

where the slopes $m$ are trivially found from (23). The first two constraints (25) and (26) describe 2-D fan shapes in $\left(\omega_{u}, \omega_{x}\right)$ and $\left(\omega_{v}, \omega_{y}\right)$, respectively. The simultaneous combination of these two constitutes a dual-fan [68]. The third constraint (27), described in [120] in the context of lens design, forces a plane's slope in $\left(\omega_{u}, \omega_{x}\right)$ to equal its slope in $\left(\omega_{v}, \omega_{y}\right)$. The surface which satisfies this third constraint is a frequency hypercone, and the surface which satisfies all three constraints we denote a hyperfan [121], because it is constructed from a fan of planes.

Knowing the frequency-domain behavior of light fields allows depth-selective filtering, either for a single depth or a range of depths, by selecting appropriately shaped frequency-planar or frequency-hyperfan passbands [121], [122]. Fig. 20 depicts a depth filtering application in which a dirty pane of glass is removed from in front of a Rainbow Lorikeet, while leaving the rest of the scene untouched. Fundamental to the hyperfan is that only a relatively small subset of the frequency domain is ever occupied by a light field. This observation is the frequency-domain manifestation of the massive redundancy in light field images, and it is the basis for an important class of noise-rejecting filters which we present below. 


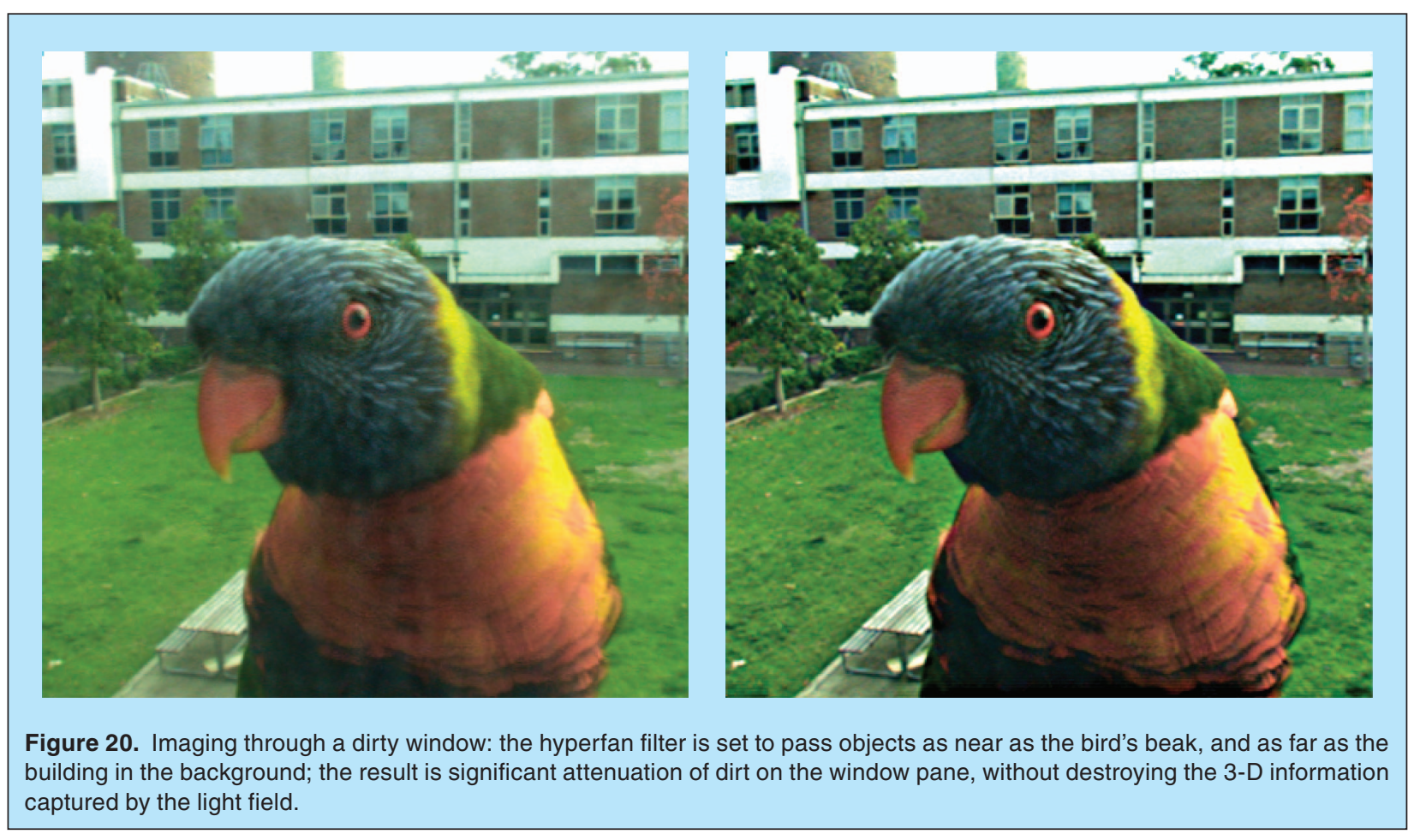

\section{Imaging in Low Light}

One of the emerging application areas for light field filtering is in difficult imaging scenarios [121], [123]. Low light, dust, fog, rain, or underwater imaging in turbid (murky) water, with suspended particulate matter, light columns and related illumination effects, all result in difficult imagery which can be problematic for modern vision algorithms. Building more robust algorithms can only go so far when the input imagery has a very unfavorable signal-to-noise ratio. The extra information measured by a light field camera, and the fact that it is represented in a manner conducive to linear filtering, means that simple methods can be employed to enhance light field imagery, even in extremely difficult scenarios.

That all light fields lie on a hyperfan ROS means that we can reject significant amounts of noise by building a filter with an appropriately shaped hyperfan passband. Two of the parameters of this passband are the nearest and farthest allowable scene elements. Because the camera never images objects at negative depths, and because many applications have a predictable maximum range-e.g., in aerial surveillance, indoor environments, or underwater imaging-we can select the passband to exclude elements beyond allowable depths.

The ideal hyperfan is a 3-D manifold, not a 4-D volume, and so practical implementation requires surrounding the hyperfan by a bandwidth. In [121], we suggest a frequency-domain passband described by:

$$
\begin{array}{r}
H_{H C}(\omega)=\exp \left(-\sqrt{2 \ln 2}\left[\frac{\left(\omega_{x} \omega_{v}-\omega_{y} \omega_{u}\right)}{\beta_{H C}^{2}}\right]^{2}\right) \\
H_{F A N}\left(\omega_{x}, \omega_{u}\right) H_{F A N}\left(\omega_{y}, \omega_{v}\right),
\end{array}
$$

where $\beta_{H C}$ is the $3-\mathrm{dB}$ bandwidth measured as the radius of the hypercone at the origin, and $H_{F A N}$ are the 2-D fans enforcing the selected depth range. Note the Gaussianlike roll off of the hypercone passband shape.

To test this passband we applied it directly in the frequency domain. This is a memory-intensive process, and points the way forward for more efficient filter implementations. Results are shown in Fig. 21(a), in which light fields available from the Stanford Light Field Archive ${ }^{1}$ were manipulated to simulate low-light image capture. This employed a model of camera noise including quantization, Poisson, Gaussian and salt \& pepper noise. The low-light images, excluding saltand-pepper noise for clarity, are depicted on the left. The noisy images (including salt-and-pepper noise) were gain-adjusted (center), and then hyperfan filtered to yield denoised images (right). The success of the method is striking, but unsurprising considering there were $17 \times 17$ samples in $n_{x}$ and $n_{y}$, yielding a very high level of redundancy. We do note that the linear hyperfan

\footnotetext{
${ }^{1}$ http://lightfield.stanford.edu/
} 


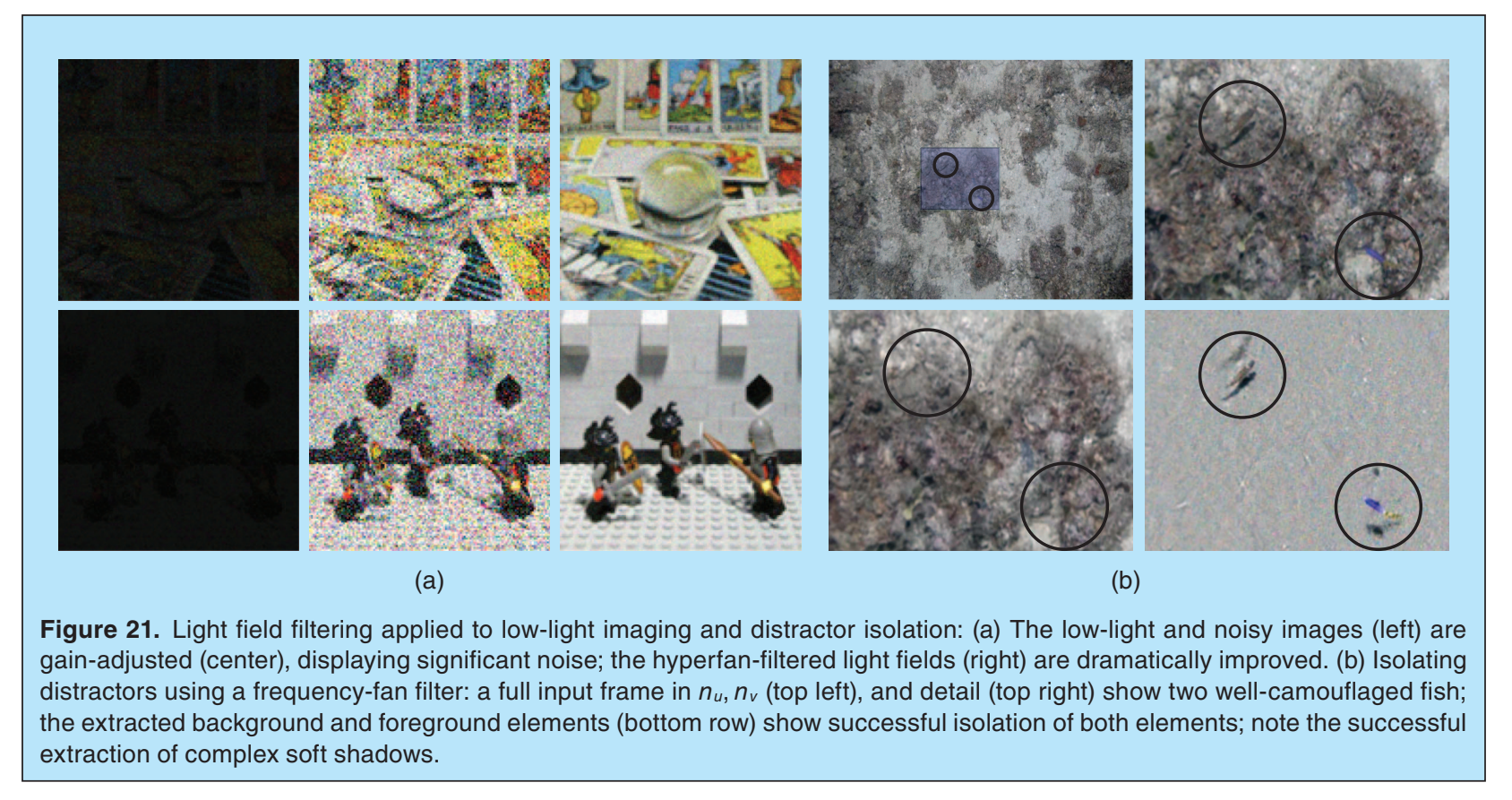

filter outperformed all competing methods, including a number of sophisticated linear and nonlinear image and video denoising methods [121].

The denoising results presented here are enabled by the massive redundancy present in the light field. As the number of spatial samples $n_{x}, n_{y}$ increases, the quality of the denoised images also increases toward a global optimum. This capability promises to be of significant interest in a range of difficult imaging scenarios.

\section{Isolating Distractors}

Low light and noise are not the only detractors of effective imagery: sometimes local residents get in the way. A common use case for computer vision is to monitor a location for change over time. Take, for example the long-term benthic monitoring program within Australia's Integrated Marine Observation System (IMOS). In this program, sites of ecological significance are revisited annually, and the resulting imagery compared to establish how the benthic habitat is changing [124]. The imagery is collected using the Australian Centre for Field Robotics (ACFR)'s Sirius autonomous under-water vehicle (AUV), and stitched into geo-referenced mosaics using tools developed at the ACFR. Putting aside the obvious technical hurdles of building geo-referenced mosaics in a GPS-denied environment, a further problem arises in performing change detection is the presence moving elements in the benthos-swimming fish, swaying algae, and so on. These cause false positives in the change detection, and so a means of removing dynamic elements from a static scene is desirable.
The problem with removing dynamic elements in this scenario is that the camera platform is in constant motion, and so all scene elements will have apparent motion relative to the camera. Given the unstructured, 3-D environments in which the imagery is collected, this motion will be non-constant, with different parts of the scene moving at different apparent velocities across the image plane. Identifying those scene elements that are genuinely moving relative to the scene can therefore be very difficult.

In [125], a method for isolating distractors from background was proposed based on the frequency-domain light field characteristics described above. The method works by building a light field from images taken over a regular spatial grid, but at different times. Such a sequence of images results, for example, from a linear, constant-velocity AUV trajectory. The resulting light field is limited in that one of the spatial dimensionsorthogonal to the direction of AUV travel-has only one sample. Regardless of this limitation, the static and dynamic elements of a scene are easily separated because the dynamic elements break the rules of parallax, and will not generally lie within the expected frequency-fan ROS of the light field. Devising a filter to reject dynamic elements is therefore similar to the noise-rejecting filter described above. Example results are shown in Fig. 21(b), in which a scene containing two moving distractors is filtered to remove the distractors and reveal the static scene. A useful consequence of the linearity of the filter is that its inverse is very easily implemented, and intuitively performs the inverse task of extracting the distractors. The result, as seen in the 
bottom right of the figure, includes isolation of soft shadows, a task which is difficult to accomplish using more conventional methods.

\section{E. Underwater Field Trials}

The E/V Nautilus is a 64-meter research vessel operated by the Ocean Exploration Trust under the direction Dr. Robert Ballard [126]. Nautilus is equipped with the remotely operated vehicles (ROVs) Hercules, Argus, Diana, and Echo, and undertakes a range of scientific research including marine geology, archaeology and biology. During the 2012 field season, a light field camera was deployed on the ROV Hercules, and collected a range of imagery over sites of predominantly archaeological interest.
Imaging a shipwreck hundreds of meters beneath the surface of the Aegean Sea, the light gathering ability of the camera was put to the test. At such depths, all illumination is artificial, provided by lamps mounted on the ROV. Because turbid water scatters light back towards the camera, adding light is not always a practical way to improve imagery. This makes the low-light and denoising capabilities of the light field camera particularly appealing. As part of the field trial, a monochrome light field camera was operated in parallel with conventional color and monochrome cameras. The key difference is that the light field camera was operated with a fraction of the illumination-roughly $40 \%$. Referring to Fig. 22, the light field camera was able to gather useful imagery
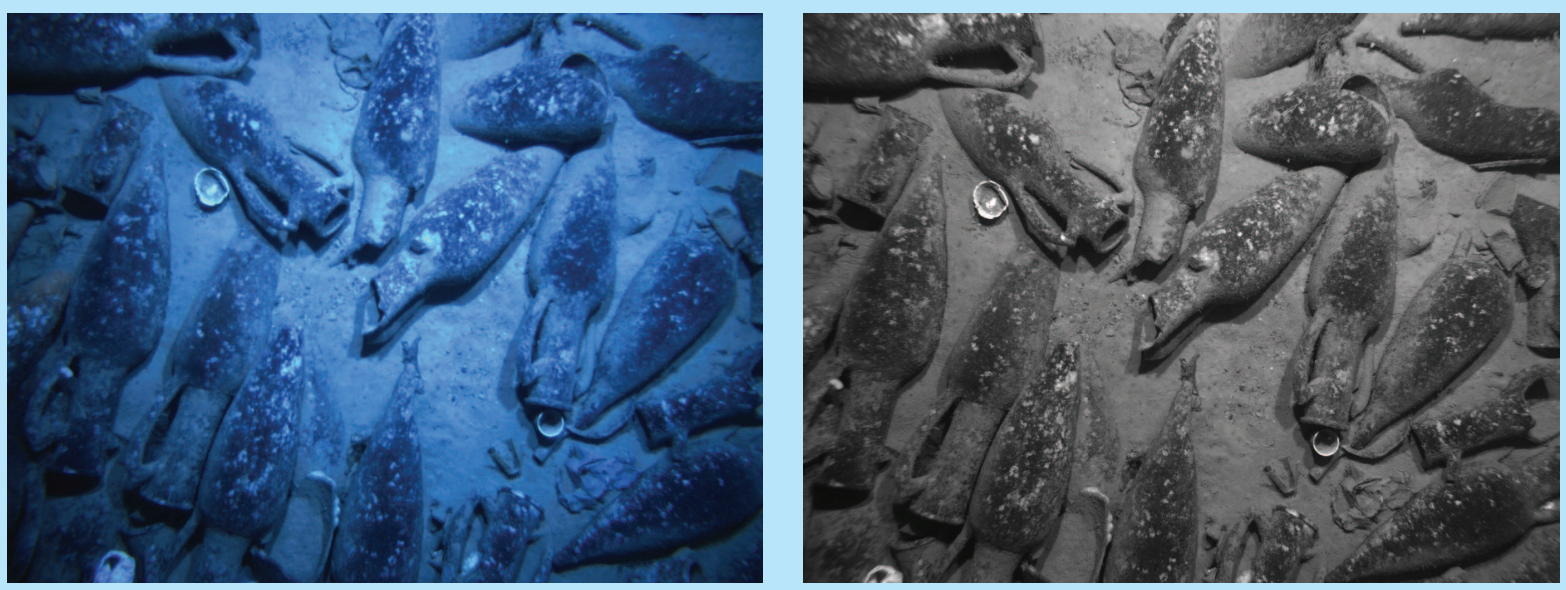

(a)
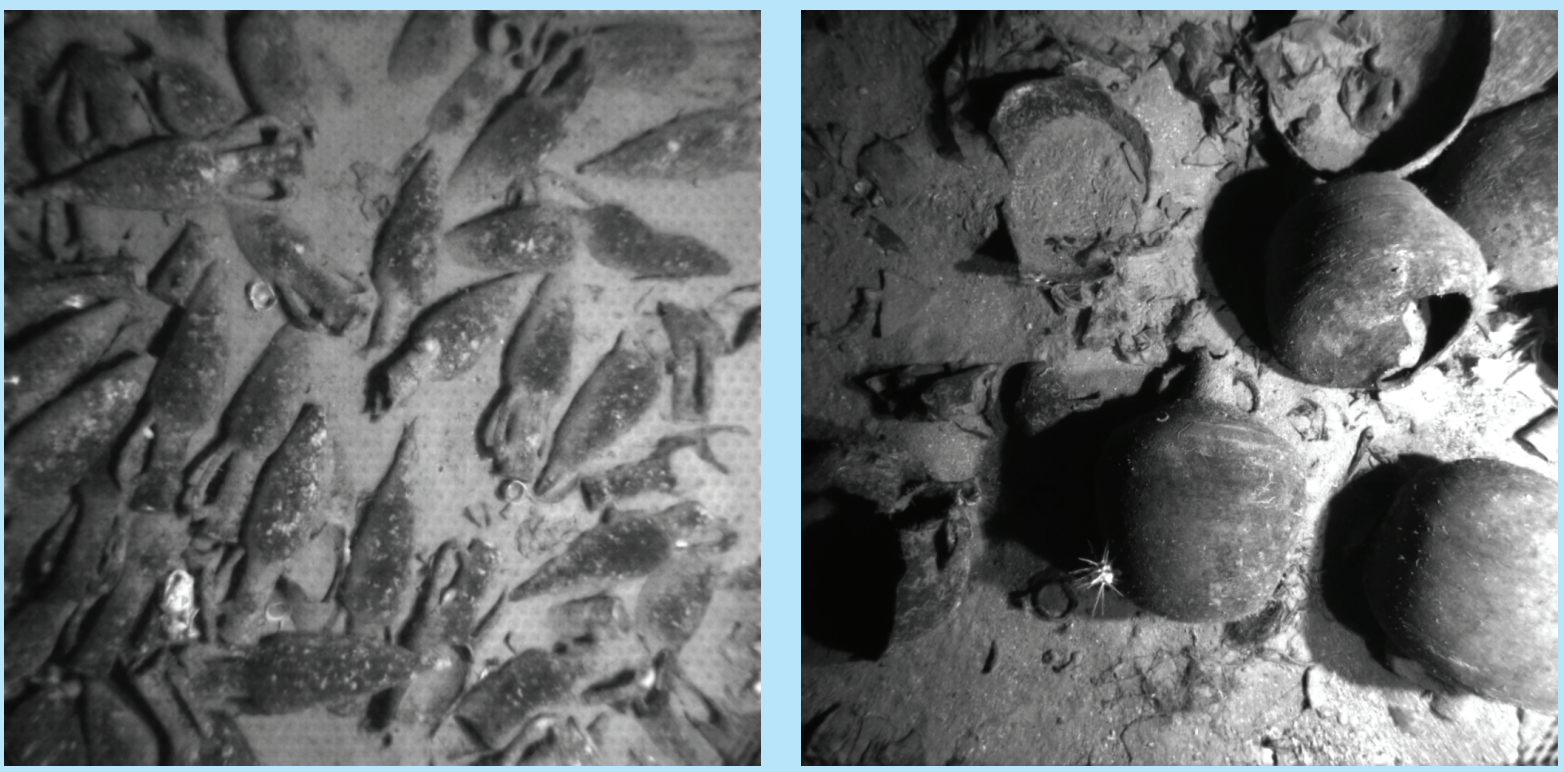

(b)

Figure 22. Images taken of shipwrecks in the Aegean Sea show the potential of light field cameras to facilitate underwater imaging. (a) Conventional color and monochrome cameras require significantly more illumination than the light field camera (b)roughly $40 \%$ the illumination was required for the light field imagery. 
that compared well with the conventional imagery, but under a fraction of the illumination. We expect this to present a significant advantage in reducing illumination power budgets, increasing imaging ranges, or in imaging through attenuating media, such as murky water.

\section{F. Future Applications}

Light field cameras allow techniques from MDSP to deliver high-quality results in constant runtime, and with a very high immunity to noise not generally shared by more complex approaches. The closed-form nature of many of these solutions makes them particularly amenable to hardware implementation, and recent advances in hardware-based light field filters [127], [128] point the way forward for fully integrated light field camera solutions. Considering the impressive light gathering, depth filtering, denoising and distractor isolating capabilities that arise naturally from light field imagery, it seems inevitable that these cameras will find their way into a wide range of difficult imaging applications.

\section{Conclusion}

We briefly reviewed the fundamental theory of MD filter design with the intention of giving the reader an insightful overview of some of the exciting new theory and applications that are emerging in the field of MD circuits and systems. Excellent texts, such as [23], provide a thorough description of the topic. Here, our emphasis has been the use of the MD Laplace transform, which is not thoroughly treated elsewhere, as a bridge in design between the continuous- and discrete-domains. Further, we emphasized the use of the first-order "frequencyplanar" MD transfer function as an important pseudopassive stable IIR building block for making higher-order MD IIR filters. Some emerging potential applications of spatio-temporal MD FIR filters were also described. First, in for cognitive radio systems and, second, for synthetic aperture systems in radio astronomy. In both cases, the use of real-time 2-D/3-D FIR filters is described. We further emphasized VLSI circuit implementations of digital and analog circuits for the 2-D and 3-D IIR spatiotemporal case, where the ultimate objective is to make real-time beamformers with high directional-selectivity, high-throughput, low-latency and low-complexity. Recently published systolic architectures are described that are based on frequency-planar MD prototypes, and yield an especially fast throughput of one complete spatial frame of data per clock cycle (the so-called OFPCC method), for single phase digital systems, and multiple frames per clock cycle (MFPCC) for polyphase systems. These polyphase MD circuits are very suitable for use with time-interleaved analog-to-digital converters that operate at several dozen $\mathrm{GHz}$ these days. Finally, we ended our discussion with a description of novel approaches by which the 4-D signals from a light field camera have been enhanced using 4-D hyperfan filters with specific applications in low-light imaging, depth filtering, denoising and the isolation of distracting objects. Applications in digital computational photography, sensing and robotics were described.

\section{Acknowledgments}

Arjuna Madanayake gratefully acknowledges financial support from the US National Science Foundation (NSF), grant \# 1247940 EARS (Andrew Clegg and George Haddad), Office of Naval Research (ONR) Code 312 Communications Division, grant \# N000141310079 (Santanu Das and Douglas Crowder), the College of Engineering, University of Akron, USA, iCORE, and The University of Calgary, Alberta, Canada for financial support. Chamith Wijenayake thanks the University of Akron, USA for financial support. Len Bruton and Thushara Gunaratne gratefully acknowledge financial support from NSERC Canada and the Institute of Space Imaging Science (ISIS). Donald G. Dansereau and Stefan B. Williams are grateful for financial support from the Australian Research Council (ARC), Australia's Integrated Marine Observing System (IMOS) funded through the Department of Industry, Innovation, Science, Research and Tertiary Education (DIISRTE), the University of Sydney's International Postgraduate Research Scholarship scheme and the Ocean Exploration Trust E/V Nautilus program.

\section{Appendix}

A review of 2-D, 3-D and 4-D plane waves. Consider the case of three dimensional plane waves as an example. Such a plane wave can be written in the form

$$
w_{a}\left(t_{1}, t_{2}, t_{3}\right)=w_{W \text { front }}\left(d_{1} t_{1}+d_{2} t_{2}+d_{3} t_{3}\right) \equiv w_{W \text { front }}\left(\mathbf{d}^{T} \mathbf{t}\right),
$$

where we have, for compactness, represented the 3 -tuples as oriented row vectors $\mathbf{d} \equiv\left[d_{1} d_{2} d_{3}\right]^{T}$ and $\mathbf{t} \equiv\left[t_{1} t_{2} t_{3}\right]^{T}$. Without loss of generality, we select $\mathrm{d}$ as a unit vector. By simple geometry, $d_{1} t_{1}+d_{2} t_{2}+d_{3} t_{3}=\mu$ is a 3 -D plane in $\mathbb{R}^{3}$ having unit normals $\pm \mathbf{d}$ and is perpendicular distance $\mu$ from the origin. Then, over all real $\mu$, (29) is an infinite set of parallel planes (one for each value of $\mu$ ) and in each such plane the value of (29) is simply $w_{\text {Wave-Front }}(\mu)$. The 1-D function $w_{W \text { front }}(\cdot)$ is the so-called "wave front" of the 3-D plane wave. Note that a plane wave has an ROS that is of infinite-extent in $\mathbb{R}^{3}$. Therefore, in practice, such ideal plane waves do not exist; they are always windowed in both space and time (i.e., apertured) and spatially-sampled. The 4-D version of (29) is a hyper-plane in $\left(t_{1}, t_{2}, t_{3}, t_{4}\right) \in \mathbb{R}^{4}$ and may be 
visualized as a continuum of parallel 3-D plane waves in $\left(t_{1}, t_{2}, t_{3}\right) \in \mathbb{R}^{3}$, where there exists one 3 -D plane wave for each value of $t_{4}$. The distance of the that 3 -D plane wave from the origin varies linearly as a function of $t_{4}$.

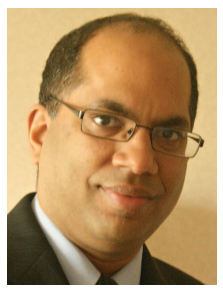

Arjuna Madanayake is an assistant professor of electrical and computer engineering (ECE) at the University of Akron, Ohio, USA, and an adjunct assistant professor at the department of ECE, University of Calgary, Alberta, Canada. He completed the Ph.D. in Electrical Engineering, at the University of Calgary, in 2008, and was a post doctoral associate at the Multi-Dimensional Signal Processing group and ISIS, University of Calgary, from March 2008 to November 2009. He completed his Masters degree at the University of Calgary in 2004, and a B.Sc. degree in Electronic and Telecommunication Engineering, from the University of Moratuwa, Sri Lanka, in 2001, with First Class Honours. Arjuna has won an NSERC Post Doctoral Fellowship Award in 2009 where he was chosen as the most outstanding candidate in electrical engineering and computing science by the NSERC selection committee. The award was gratefully declined. Arjuna Madanayake also won the iCORE International Doctoral Award for graduate studies in 2005 and the Deans Entrance Scholarship (2004) for doctoral work. Dr Madanayake's research interests include MD circuits, systems and signal processing, RF antenna and active circuits, analog CMOS circuits, digital VLSI and computer architecture. He lives in Akron, Ohio, USA with his family.

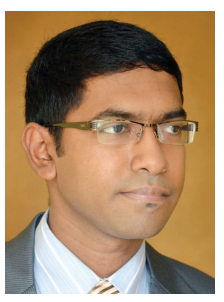

Chamith Wijenayake is a Ph.D. candidate at the department of ECE at the University of Akron. He completed his B.Sc. degree in Electronic and Telecommunication Engineering with first class honors from the University of Moratuwa, Sri Lanka in 2007. Prior to doctoral studies Chamith Wijenayake was a telecommunication engineer at HUAWEI technologies Lanka pvt. Ltd and also an instructor at the University of Moratuwa. Chamith's research interests include multidimensional analog/digital and mixed signal processing, smart antenna array signal processing, FPGA based system design, digital VLSI.

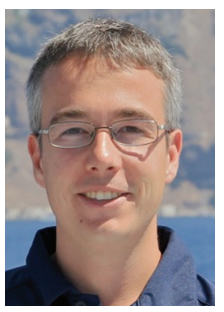

Donald G. Dansereau completed B.Sc. and M.Sc. degrees in electrical and computer engineering at the University of Calgary in 2001 and 2004, receiving the Governor Generals Gold Medal for his Master's work. His industry experience includes work on real-time physics engines, structured-light wafer bump metrology, and automated test equipment for high-speed chip interfaces. Donald is presently completing his doctoral work on light field imaging in challenging environments at the Australian Centre for Field Robotics, University of Sydney, Australia. His recent field work includes marine archaeology on a sunken Bronze Age city in Greece, seamount mapping and hydrothermal vent exploration in the Sea of Crete and Aeolian Arc, mapping benthic habitats off the coast of Tasmania, and hydrochemistry and wreck exploration in Lake Geneva.

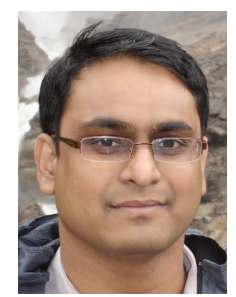

Thushara K. Gunaratne (S'06) was born in Colombo, Sri Lanka. In 2004, he received the B.Sc. Engineering degree in Electronics and Telecommunication Engineering from the University of Moratuwa, Sri Lanka. In 2007 and 2011, he received the M.Sc. and Ph.D. degrees in Electrical Engineering from the University of Calgary, Calgary, Canada, respectively. In 2003, he won seven awards including the UNESCO Team Gold Medal for the most outstanding academic performance in his undergraduate studies in the Faculty of Engineering, University of Moratuwa, Sri Lanka. During his tenure as a graduate student in the department of ECE, University of Calgary (20042011), he won number of scholarships, awards and travel grants including the MICRONET Scholarship, Honor Mentioned Student Author-IEEE ISCAS 2007 and University Research Services Travel Grant. He is currently working as a postdoctoral research fellow in Electrical Engineering at the University of Calgary, Calgary, Canada in the area of MD digital signal processing systems and circuits. His research interests include broadband beamforming techniques for applications in radio astronomy, remote sensing and high performance computer systems.

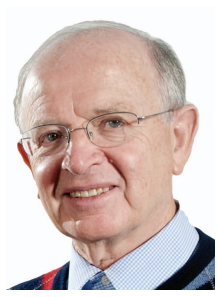

Leonard T. Bruton is a Faculty Professor of ECE at the University of Calgary, Alberta, Canada, and an Adjunct Professor in Electrical and Computer Engineering at the University of Victoria, Victoria, B.C., Canada. He carries out research in the fields of analog and digital signal processing with emphasis on MD circuits and systems and he is especially interested in the emerging applications of 2-D, 3-D and 4-D filters for real-time directional filtering, including applications in beamforming and digital image processing. He is the inventor of a number of widely used microelectronic filtering methods, including the class of frequency dependent negative resistance (FDNR) analog filters and the class of Lossless Discrete Integrator (LDI) discrete-domain filters. He holds a number of patents. 
He is a Life Fellow of the IEEE, a member of the Royal Society of Canada, a recipient of the 2002 Education Award of the IEEE Circuits and Systems Society, the 2007 Technical Achievement Award of the IEEE Circuits and Systems Society, the 50th Jubilee Medal of the IEEE Circuits and Systems Society and the 1994 Outstanding Engineer Award of IEEE Canada (Region 7) with the citation "For leadership in engineering and the engineering profession and for research in the field of microelectronic digital and analog real-time filter design." In Canada, he received the 1992 Manning Principal Award for Innovation and he is one of 162 scientists selected for inclusion in the textbook Great Canadian Scientists by Barry Shell, Polestar Book Publishers, 1997 (ISBN 1-896095-36-4). He received the 1992 Alberta Science and Technology Award (ASTech) for Innovation in Science in recognition of his leadership in science and in 1993 he received the Federal Government of Canada's 125th Anniversary of Canadian Confederation Medal in recognition of his significant contributions to compatriots, community and to Canada. He is a recipient of the Centennial Award from the Alberta Association of Professional Engineers, Geologists and Geophysicists of Alberta. He has held the positions of Head of Electrical Engineering, Dean of Engineering and Vice-President Research at the University of Calgary and was the founding Dean of Engineering at the University of Victoria.

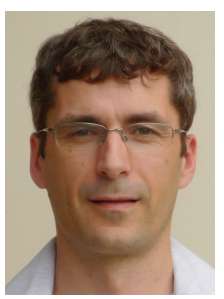

Stefan B. Williams is an Associate Professor in the University of Sydney's School of Aerospace, Mechanical and Mechatronic Engineering. He is a member of the Australian Centre for Field Robotics where he leads the Marine Robotics group. He is also the head of Australia's Integrated Marine Observing System AUV Facility. His research interests include Simultaneous Localization and Mapping in unstructured underwater environments, autonomous navigation and control and classification and clustering of large volumes of data collected by robotic systems. He received his Ph.D. from the University of Sydney in 2002 and completed a Bachelor of Applied Science with first class honours in 1997 at the University of Waterloo, Canada.

\section{References}

[1] D. Dudgeon and R. Mersereau, Multidimensional Digital Signal Processing. Englewood Cliffs, NJ: Prentice-Hall, 1990, ISBN: 978-0132276382. [2] N. K. Bose, Multidimensional Systems: Theory and Applications, 2nd ed. New York: Springer, 2003, ISBN: 9781402016233.

[3] N. Devroye, M. Vu, and V. Tarokh, "Cognitve radio networks," IEEE Signal Process. Mag., vol. 25, no. 6, pp. 12-23, Nov. 2008.

[4] E. Hossain, L. Le, N. Devroye, and M. Vu, Cognitive Radio Communications and Networks; Principles and Practice, V. Tarokh, I. F. Blake, and A. Gulliver, Eds. Elsevier, 2009, ch. 4.

[5] B. Wilburn, M. Smulski, H. K. Lee, and M. Horowitz, "The light field video camera," in Proc. Media Processors, SPIE Electronic Imaging, 2002.
[6] M. Levoy and P. Hanrahan, "Light field rendering," in Proc. 23rd Annu. Conf. Computer Graphics and Interactive Techniques, 1996, pp. 31-42.

[7] P. Dewdney, P. Hall, R. Schilizzi, and T. Lazio, "The square kilometre array," Proc. IEEE, vol. 97, no. 8, pp. 1482-1496, Aug. 2009.

[8] D. R. DeBoer and D. C. J. Bock, "The allen telescope array: Splitting the aperture," IEEE Microwave Mag., vol. 5, no. 2, pp. 46-53, June 2004. [9] "Workshop on Enhancing Access to the Radio Spectrum (EARS)," NSF, Arlington, VA, Final Report, Aug. 2010.

[10] C. Wijenayake, A. Madanayake, and L. T. Bruton, "Broadband multiple cone-beam 3D IIR digital filters applied to planar dense aperture arrays," IEEE Trans. Antennas Propagat., vol. 60, no. 11, pp. 5136-5146, Nov. 2012

[11] A. Walther, "Radiometry and coherence," J. Opt. Soc. Amer., vol. 58, pp. 1256-1259, 1968.

[12] N. K. Bose, "Feature-multidimensional systems and signal processing: Good theory for good practice," IEEE Circuits Syst. Mag., vol. 4, pp. 20-41, 2007.

[13] Z. Lin, "Feedback stabilization of multivariable two-dimensional linear systems," Int. J. Control, vol. 48, no. 3, pp. 1301-1317, 1988.

[14] Z. Lin, L. T. Bruton, and N. R. Bartley, "Design of highly selective two-dimensional recursive fan filters by relaxing symmetry constraints," Electron. Lett., UK, vol. 24, no. 22, pp. 1361-1362, 1988.

[15] Z. Lin and L. T. Bruton, "BIBO stability of inverse 2-D digital filters in the presence of nonessential singularities of the second kind," IEEE Trans. Circuits Syst., vol. 36, no. 2, pp. 244-254, 1989.

[16] Z. Lin, J. Lam, K. Galkowski, and S. Xu, "A constructive approach to stabilizability and stabilization of a class of nD systems," Multidimensional Syst. Signal Process., vol. 12, no. 3, pp. 329-343, 2001.

[17] K. Galkowski, S. Xu, J. Lam, and Z. Lin, "LMI approach to statefeedback stabilization of multidimensional systems," Int. J. Control, UK, vol. 76, pp. 1428-1436, 2003.

[18] V. Ramachandran, G. S. Takhar, and M. N. S. Swamy, "On the synthesis of a class of multivariable positive real functions," Proc. IEEE, vol. 65 , no. 6 , pp. 976-978, 1977.

[19] M. Ahmad, C. Reddy, V. Ramachandran, and M. Swamy, "A class of multivariable positive real functions realizable by resistively terminated lossless ladder networks," IEEE Trans. Circuits Syst., vol. 26, no. 8, pp. 659-662, 1979.

[20] [Online]. Available: http://www-mddsp.enel.ucalgary.ca/People/ bruton/research.htm

[21] [Online]. Available: http://www.aspc-lab.net

[22] [Online]. Available: http://www.csr.acfr.usyd.edu.au/people/ StefanWilliams.htm

[23] H. Schroder and H. Blume, One- and Multidimensional Signal Processing, Algorithms and Applications in Image Processing. New York: Wiley, 2000, ISBN: 0471805416.

[24] L. Khademi and L. T. Bruton, "Reducing the computational complexity of narrowband 2D fan filters using shaped 2D window functions," in IEEE Int. Symp. Circuits and Systems, Bangkok, Thailand, May 2003, vol. 3, pp. 702-705.

[25] A. Madanayake and L. T. Bruton, "First-order frequency-planar module for fan, beam, and cone plane-wave filters," IEEE Trans. Circuits Syst. II, Exp. Briefs, vol. 53, no. 8, pp. 697-701, Aug. 2006.

[26] M. Sid-Ahmed, "Two-dimensional analog filters: A new form of realization," IEEE Trans. Circuits Syst., vol. 36, no. 1, pp. 153-154, Jan. 1989. [27] M. A. Sid-Ahmed and H. J. Kaufman, "2-D analog filters for real-time video signal processing," IEEE Trans. Consum. Electron., vol. 36, no. 2, pp. 138-141, May 1990.

[28] R. K. Bertschmann, N. R. Bartley, and L. T. Bruton, "A 3D integratordifferentiator double-loop (IDD) filter for raster-scan video processing," in IEEE Int. Symp. Circuits and Systems, May 1995, pp. 470-473.

[29] L. T. Bruton, "2D/3D analog mixed-domain beam/fan filters-internal report," Univ. Calgary, Calgary, Calgary, AB, Canada, Tech. Rep., 2003.

[30] M. Maini, "2D spatio-temporal recursive filters for plane wave array processing," M.Sc. dissertation, Univ. Calgary, Calgary, AB, Canada, 2006.

[31] P. A. Ramamoorthy and L. T. Bruton, "Design of stable twodimensional analogue and digital filters with application in image processing," Circuit Theory Appl., vol. 7, pp. 229-245, 1979.

[32] P. Ramamoorthy and L. Bruton, Selected Papers in Multidimensional Signal Processing. Piscataway, NJ: IEEE Press, 1986, ch. Design of Stable Two-Dimensional Analog and Digital Filters with Applications in Image Processing, pp. 15-32. 
[33] L. T. Bruton, A. Madanayake, C. Wijenayake, and M. Maini, "Continuous-time analog two-dimensional IIR beam filters," IEEE Trans. Circuits Syst. II, Exp. Briefs, vol. 59, no. 7, pp. 419-423, July 2012.

[34] L. T. Bruton and Y. Zhang, "Applications of lossless MD structures for the filtering of digital image sequences," in Int. Symp. Circuits and Systems, Singapore, June 1991, pp. 128-131.

[35] Y. J. Zhang and L. T. Bruton, "Applications of 3D LCR networks in the design of 3D recursive filters," in IEEE Int. Symp. Circuits and Systems, Chicago, IL, USA, May 1993, pp. 906-909.

[36] Y. J. Zhang and L. T. Bruton, "Differentiator-type three-dimensional recursive ladder filters having frequency planar or frequency beam shaped passbands," IEEE Trans. Circuits Syst. Video Technol., vol. 2, no. 3, pp. 297-305, Sept. 1992.

[37] Y. Zhang and L. T. Bruton, "Applications of 3D LCR networks in the design of 3D recursive filters for processing image sequences," IEEE Trans. Circuits Syst. Video Technol., vol. 4, no. 4, pp. 369-382, Aug. 1994. [38] L. T. Bruton and T. E. Strecker, "Two dimensional discrete filters using spatial integrators," Proc. IEEE, vol. 130, pp. 271-275, Dec. 1983.

[39] L. T. Bruton and N. R. Bartley, "Highly selective three-dimensional recursive beam filters using intersecting resonant planes," IEEE Trans. Circuits Syst., vol. 30, no. 3, pp. 1048-1055, Mar. 1983.

[40] D. Jin and L. T. Bruton, "Closed-form impulse responses of discretedomain multidimensional filters," IEEE Trans. Circuits Syst. II, Analog Digit. Signal Process., vol. 42, no. 2, pp. 607-609, Sept. 1995.

[41] P. Agathoklis and L. T. Bruton, "Practical BIBO-stability of n-dimensional discrete systems," Proc. IEEE, vol. 130, pp. 236-242, Dec. 1983.

[42] P. A. Ramamoorthy and L. T. Bruton, Topics in Applied Physics: Two Dimensional Digital Signal Processing I, Linear Filters. New York: Springer-Verlag, vol. 42, 1981, ch. Design of Two-Dimensional Recursive Filters.

[43] M. S. Lazar and L. T. Bruton, "On the practical BIBO stability of multidimensional filters," in IEEE Int. Symp. Circuits and Systems, May 1993, pp. 571-574

[44] P. A. Ramamoorthy and L. T. Bruton, "Design of stable 2-dimensional discrete recursive filters," Electron. Lett., vol. 12, no. 25, pp. 659-660, Dec. 1976

[45] Q. Liu and L. Bruton, "Design and implementation of 3-D recursive digital filters by applying spectral transformations to LC filter networks," in Int. Symp. Circuits and Systems, Helsinki, Finland, 1988, pp. 1261-1264.

[46] Z. Q. Liu, L. T. Bruton, J. Bezdek, J. Keller, S. Dance, N. Bartley, and C. S. Zhang, "Dynamic image sequence analysis using fuzzy measures," IEEE Trans. Syst. Man, Cybern. B, vol. 31, no. 4, pp. 557-572, Aug. 2001.

[47] L. T. Bruton and N. R. Bartley, "Multidimensional network resonance and Q-factor applied to image processing," in IEEE Int. Symp. Circuits and Systems, May 1983, pp. 398-401.

[48] L. T. Bruton and N. R. Bartley, "The design of highly-selective adaptive three-dimensional recursive cone filters," in IEEE Int. Symp. Circuits and Systems, May 1987, pp. 972-975.

[49] L. T. Bruton, N. R. Bartley, and Z. Q. Liu, "On the classification of moving objects in image sequences using $3 \mathrm{D}$ adaptive recursive tracking filters and neural networks," in Asilomar Conf. Signals, Systems and Computers, Nov. 1995, vol. 2, pp. 1006-1010.

[50] C. J. Kulach, L. T. Bruton, and N. Bartley, "A real-time video implementation of a three-dimensional first-order recursive discrete-time filter," in IEEE Int. Symp. Circuits and Systems, May 1996, vol. 2, pp. 699-702. [51] L. T. Bruton and N. R. Bartley, "Three dimensional image processing using the concept of network resonance," IEEE Trans. Circuits Syst., vol. 32 , no. 7 , pp. $664-672,1985$.

[52] L. T. Bruton and N. R. Bartley, "The enhancement and tracking of moving objects in digital image using adaptive three-dimensional recursive filters," IEEE Trans. Circuits Syst., vol. 33, no. 6, pp. 604-613, June 1986.

[53] L. B. Hewitt, L. T. Bruton, and N. Bartley, "A portable high-speed digital image processing instrument for the enhancement of television images," IEEE Trans. Instrum. Meas., vol. 35, no. 4, pp. 596-605, Dec. 1986.

[54] B. Kuenzle and L. T. Bruton, "3D IIR filtering using decimated DFT-polyphase filter bank structures," IEEE Trans. Circuits Syst., Part I, vol. 53, no. 2, pp. 394-408, Feb. 2006.

[55] T. Gunaratne and L. T. Bruton, "Beamforming of broad-band bandpass plane waves using polyphase 2D FIR trapezoidal filters," IEEE Trans. Circuits Syst. I, Reg. Papers, vol. 55, pp. 838-850, 2008.
[56] A. Madanayake, T. K. Gunaratne, and L. T. Bruton, "Massivelyparallel systolic-array architectures for 2D IIR polyphase space-time plane-wave beam digital filters," Int. J. Circuit Theory Appl., vol. 40, pp. $455-475,2010$

[57] D. G. Dansereau and L. T. Bruton, "A 4D frequency-planar IIR filter and its application to light field processing," in Proc. Int. Symp. Circuits and Systems, May 2003, vol. 4, pp. 476-479.

[58] L. T. Bruton, "A 3D polyphase-DFT cone filter bank for broad band plane wave filtering," in Int. Symp. Circuits and Systems, Vancouver, BC, Canada, May 2004, vol. 3, pp. 181-184.

[59] B. Kuenzle and L. T. Bruton, "A novel low-complexity spatio-temporal ultra wide-angle polyphase cone filter bank applied to sub-pixel motion discrimination," in IEEE Int. Symp. Circuits and Systems, Kobe, Japan, May 2005, vol. 3, pp. 2397-2400.

[60] N. Rajapaksha, A. Madanayake, and L. T. Bruton, "2D spacetime wavedigital multi-fan filter banks for signals consisting of multiple plane waves," Multidimensional Syst. Signal Process., vol. 0923-6082, pp. 1-23, 2012.

[61] A. Madanayake, N. Rajapaksha, C. Wijenayake, K. S. Lee, L. T. Bruton, and L. Belostotski, "A new class of spatially-discrete timecontinuous 2D IIR filters based on wave-digital-filter theory," in IEEE Pacific Rim Conf. Communications, Computers and Signal Processing (PacRim), Aug. 2011, pp. 685-690.

[62] Q. Liu and L. T. Bruton, "Sensitivity analysis of 3D recursive digital beam filter structures," in Asilomar Conf. Circuits, Systems, and Computers, 1988, pp. 161-165.

[63] Q. Liu and L. T. Bruton, "Design of 3D planar and beam recursive digital filters using spectral transformations," IEEE Trans. Circuits Syst., vol. 36, no. 3, pp. 365-374, Mar. 1989

[64] X. Liu and L. T. Bruton, "Parallel computing in multidimensional recursive filtering," Int. J. High Speed Electron. Syst., vol. 4, no. 2, pp. 219-243, 1993

[65] X. Liu and L. T. Bruton, "High-speed systolic ladder structures of multidimensional recursive digital filters," IEEE Trans. Signal Process. vol. 44, no. 4, pp. 1048-1055, Apr. 1996

[66] A. Madanayake and L. T. Bruton, "A systolic-array architecture for first-order 3D IIR frequency-planar filters," IEEE Trans. Circuits Syst. I, Reg. Papers, vol. 55, no. 6, pp. 1546-1559, 2008.

[67] A. Madanayake, S. V. Hum, and L. T. Bruton, "A systolic array 2D IIR broadband RF beamformer," IEEE Trans. Circuits Syst. II, Exp. Briefs, vol. 55 , no. 12 , pp. $1244-1248$, Dec. 2008

[68] D. Dansereau and L. T. Bruton, "A 4D dual-fan filter bank for depth filtering in light fields," IEEE Trans. Signal Process., vol. 55, no. 2, pp. 542-549, Feb. 2007.

[69] L. T. Bruton, "Three-dimensional cone filter banks," IEEE Trans. Circuits Syst. I, Fundam. Theory Appl., vol. 50, no. 2, pp. 208-216, Feb. 2003. [70] H. Arslan, Cognitive Radio, Software Defined Radio, and Adaptive Wireless Systems. Springer, 2007.

[71] I. Cosovic, F. K. Jondral, M. M. Buddhikot, and R. Kohno, Cognitive Radio and Dynamic Spectrum Sharing Systems. New York: Hindawi, 2008. [72] B. A. Fette and B. Fette, Cognitive Radio Technology. Newnes, 2006. [73] M. Dillinger, K. Madani, and N. Alonistioti, Software Defined Radio. Architectures, Systems and Functions. Hoboken, NJ: Wiley, 2003.

[74] J. Liberti and T. Rappaport, Smart Antennas for Wireless Communications-IS-95 and Third Generation CDMA Applications. Upper Saddle River, NJ: Prentice-Hall, 1999.

[75] H. L. V. Trees, Optimum Array Processing; Detection, Estimation and Modulation Theory, Part IV. Hoboken, NJ: Wiley, 2002.

[76] D. H. Johnson and D. E. Dudgeon, Array Signal Processing: Concepts and Techniques. Englewood Cliffs, NJ: Prentice-Hall, Feb. 1993.

[77] T. Gunaratne and L. Bruton, "Adaptive Complex-Coefficient 2D FIR trapezoidal filters for broadband beamforming in cognitive radio systems," Circuits Syst. Signal Process., vol. 30, no. 3, pp. 587-608, 2011.

[78] W. Lu and A. Antoniou, Two-dimensional Digital Filters. Boca Raton, FL: CRC Press, July 1992.

[79] A. B. Carlson, P. B. Crilly, and J. Rutledge, Communication Systems. New York: McGraw-Hill, June 2001.

[80] T. Gunaratne, L. Bruton, and P. Agathoklis, "Broadband beamforming of focal plane array (FPA) signals using Real-Time Spatio-Temporal 3D FIR frustum digital filters," IEEE Trans. Antennas Propagat., vol. PP, no. 99, p. 1, 2011.

[81] P. Hall, R. Schilizzi, P. Dewdney, and J. Lazio, "The square kilometer array (SKA) radio telescope: Progress and technical directions," Radio Sci. Bull., vol. 326, no. 326, pp. 4-19, Sept. 2008. 
[82] R. T. Schilizzi, P. E. F. Dewdney, and T. J. W. Lazio, "The square kilometre array," in Ground-based and Airborne Telescopes II, Marseille, France, 2008, p. 70.

[83] P. Dewdney, J. b. d. Vaate, K. Cloete, A. Gunst, D. Hall, R. McCool, N. Roddis, and W. Turner. (2010, Nov.). "SKA phase 1: Preliminary system description," SKA Memo 130, Tech. Rep. [Online]. Available: http://www.skatelescope.org/uploaded/21705_130_Memo_Dewdney.pdf [84] T. H. Hankins, J. S. Kern, J. C. Weatherall, and J. A. Eilek, "Nanosecond radio bursts from strong plasma turbulence in the crab pulsar," Nature, vol. 422, no. 6928, pp. 141-143, 2003.

[85] J. Fisher and R. Bradley, "Full-Sampling focal plane arrays," in Imaging Radio Through Submillimeter Wavelengths, 2000, vol. 217, p. 11.

[86] A. van Ardenne, J. Bregman, W. van Cappellen, G. Kant, and J. de Vaate, "Extending the field of view with phased array techniques: Results of european SKA research," Proc. IEEE, vol. 97, no. 8, pp. 1531-1542, 2009.

[87] D. DeBoer, R. Gough, J. Bunton, T. Cornwell, R. Beresford, S. Johnston, I. Feain, A. Schinckel, C. Jackson, M. Kesteven et al., "Australian SKA pathfinder: A high-dynamic range wide-field of view survey telescope," Proc. IEEE, vol. 97, no. 8, pp. 1507-1521, 2009.

[88] B. Veidt and P. Dewdney. (2005). "A Phased-Array feed demonstrator for radio telescopes," in Proc. General Assembly, Int. Union Radio Science (URSI) [Online]. Available: http://www.ursi.org/Proceedings/ ProcGA05/pdf/B04.10(0638).pdf

[89] P. Patel, D. Kant, E. Wal, and A. Van Ardene, "Phased array antennas demonstrator as a radio telescope-EMBRACE," in Antennas and Propagation Society Int. Symp., 2008. AP-S 2008. IEEE, 2008, pp. 1-4.

[90] S. Hay, J. O’Sullivan, J. Kot, C. Granet, A. Grancea, A. Forsyth, and D. Hayman, "Focal plane array development for ASKAP (Australian SKA pathfinder)," in Second European Conf. Antennas and Propagation (EuCAP 2007), 2007, pp. 1-5.

[91] T. K. Gunaratne, "Beamforming of broadband bandpass signals using multidimensional FIR filters," Ph.D. dissertation, Univ. Calgary, Calgary, AB, Canada, Sept. 2011.

[92] Y. Rahmat-Samii, Antenna Engineering Handbook, J. L. Volakis, Ed. New York: McGraw-Hill, 2007, pp. 15-19.

[93] N. Liyanage, L. Bruton, and P. Agathoklis, "On the attenuation of interference and mutual coupling in antenna arrays using 3D spacetime filters," in Communications, Computers and Signal Processing, 2009. PacRim 2009. IEEE Pacific Rim Conf., 2009, pp. 146-151.

[94] N. Liyanage, "3D space-time digital filtering for radio astronomy," Ph.D. dissertation, Univ. Victoria, Victoria, BC, Canada, 2009

[95] D. Jones, "Characterization of a phased array feed model," Ph.D. dissertation, Brigham Young Univ., Provo, UT, 2008.

[96] K. W. Martin, "Complex signal processing is not complex," IEEE Trans. Circuits Syst.-1, Reg. Papers, vol. 51, no. 9, pp. 1823-1836, 2004.

[97] H. Schroder and H. Blume, One-and-Multidimensional Signal Processing: Algorithms and Applications in Image Processing. Wiley, Dec. 2000 .

[98] S. Kung, "VLSI Array Processors," IEEE ASSP Mag., vol. 2, no. 3, pp. 4-22, July 1985.

[99] B. K. Mohanty and P. K. Meher, "High throughput and low-latency implementation of bit-level systolic architecture for 1D and 2D digital filters," IEE Proc. Comput. Dig. Techn., vol. 146, no. 2, pp. 91-99, Mar. 1999.

[100] A. Madanayake, T. Gunaratne, and L. Bruton, "Reducing the multiplier-complexity of massively parallel polyphase 2D IIR broadband beam filters," Circuits Syst. Signal Process., vol. 31, pp. 1229-1243, 2012. [101] S. Kondapalli, A. Madanayake, and L. T. Bruton, "Digital architectures for UWB beamforming using 2-D IIR spatio-temporal frequencyplanar filters," Int. J. Antennas Propagat., vol. 2012, p. 19, 2012.

[102] A. Madanayake and L. T. Bruton, "Low-complexity distributedparallel-processor for 2D IIR broadband beam plane-wave filters," Can. J. Elect. Comput. Eng. (CJECE), vol. 32, no. 3, pp. 123-131, 2007.

[103] S. V. Hum, A. Madanayake, and L. T. Bruton, "UWB beamforming using 2D beam digital filters," IEEE Trans. Antennas Propagat., vol. 57, no. 3, pp. 804-807, 2009.

[104] L. Liang and S. V. Hum, "Experimental characterization of UWB beamformers based on multidimensional beam filters," IEEE Trans. Antennas Propagat., vol. 59, pp. 304-309, 2011

[105] A. Madanayake, "Real-time FPGA architectures for space-time frequency-planar MDSP,” Ph.D. dissertation, Electr. Eng., Univ. Calgary, Calgary, AB, Canada, 2008.
[106] R. M. Joshi, A. Madanayake, J. Adikari, and L. T. Bruton, "Synthesis and array processor realization of a 2D IIR beam filter for wireless applications," IEEE Trans. Very Large Scale Integr. (VLSI) Syst., vol. 20, no. 12 , pp. $2241-2254,2012$

[107] A. Fettweis, "Wave digital filters: Theory and practice," Proc. IEEE, vol. 74, pp. 270-327, 1986 .

[108] A. Fettweis, "Multidimensional wave-digital principles: From filtering to numerical integration," in Proc. IEEE Int. Conf. Acoustics, Speech and Signal Processing, Apr. 1994, vol. 6, pp. 173-181.

[109] Y. T. Zheng, M. Zhao, Y. Song, H. Adam, U. Buddemeier, A. Bissacco, F. Brucher, T. S. Chua, and H. Neven, "Tour the world: Building a webscale landmark recognition engine," in IEEE Conf. Computer Vision and Pattern Recognition (CVPR), 2009, pp. 1085-1092.

[110] J. Shotton, A. Fitzgibbon, M. Cook, T. Sharp, M. Finocchio, R. Moore, A. Kipman, and A. Blake, "Real-time human pose recognition in parts from single depth images," in IEEE Conf. Computer Vision and Pattern Recognition (CVPR), 2011, pp. 1297-1304.

[111] T. Whelan, M. Kaess, M. Fallon, H. Johannsson, J. Leonard, and J. McDonald, "Kintinuous: Spatially extended kinectfusion," in 3rd RSS Workshop RGB-D: Advanced Reasoning Depth Cameras, July 2012, vol. 1 .

[112] A. Prusak, O. Melnychuk, H. Roth, and I. Schiller, "Pose estimation and map building with a time-of-flight-camera for robot navigation," Int. J. Intell. Syst. Technol. Appl., vol. 5, no. 3, pp. 355-364, 2008.

[113] K. Lai, L. Bo, X. Ren, and D. Fox, "Sparse distance learning for object recognition combining rgb and depth information," in IEEE Int. Conf. Robotics and Automation (ICRA), 2011, pp. 4007-4013.

[114] B. Wilburn, N. Joshi, V. Vaish, E. Talvala, E. Antunez, A. Barth, A. Adams, M. Horowitz, and M. Levoy, "High performance imaging using large camera arrays," ACM Trans. Graph., vol. 24, no. 3, pp. 765-776, 2005. [115] R. Ng, M. Levoy, M. Brédif, G. Duval, M. Horowitz, and P. Hanrahan, "Light field photography with a hand-held plenoptic camera," Comput. Sci. Tech. Rep. CSTR, vol. 2, 2005.

[116] A. Lumsdaine and T. Georgiev, "The focused plenoptic camera," in IEEE Int. Conf. Computational Photography, 2009, pp. 1-8.

[117] D. Lanman, "Mask-based light field capture and display," Ph.D. dissertation, Brown Univ., 2012.

[118] Z. Xu, J. Ke, and E. Y. Lam, "High-resolution lightfield photography using two masks," Optics Exp., vol. 20, no. 10, pp. 10 971-10 983, 2012.

[119] R. C. Bolles, H. H. Baker, and D. H. Marimont, "Epipolar-plane image analysis: An approach to determining structure from motion," Int. J. Comput. Vis., vol. 1, no. 1, pp. 7-55, 1987.

[120] A. Levin, S. W. Hasinoff, P. Green, F. Durand, and W. T. Freeman, "4D frequency analysis of computational cameras for depth of field extension," ACM Trans. Graph., vol. 28, no. 3, p. 97, 2009.

[121] D. G. Dansereau, D. L. Bongiorno, S. B. Williams, and O. Pizarro, "Light field image denoising using a linear 4D frequency-hyperfan allin-focus filter," SPIE Comput. Imaging, to be published.

[122] R. Ng, "Fourier slice photography," ACM Trans. Graph., vol. 24, no. 3, pp. 735-744, July 2005.

[123] C. Roman, G. Inglis, I. Vaughn, C. Smart, D. G. Dansereau, D. Bongiorno, M. Johnson-Roberson, and S. Williams, "New tools and methods for precision sea floor mapping," Oceanogr. Suppl., to be published.

[124] S. B. Williams, O. R. Pizarro, M. V. Jakuba, C. R. Johnson, N. S. Barrett, R. C. Babcock, G. A. Kendrick, P. D. Steinberg, A. J. Heyward, P. J. Doherty, I. Mahon, M. Johnson-Roberson, D. Steinberg, and A. Friedman, "Monitoring of benthic reference sites: Using an autonomous underwater vehicle," in IEEE Robotics and Automation Magazine. IEEE, 2012, vol. 19, no. 1, pp. 73-84.

[125] D. G. Dansereau and S. B. Williams, "Seabed modeling and distractor extraction for mobile AUVs using light field filtering," in IEEE Int. Conf. Robotics and Automation (ICRA), May 2011, pp. 1634-1639.

[126] K. Bell, K. Elliott, C. Martinez, and S. Fuller, "New frontiers in ocean exploration: The E/V nautilus and NOAA ship okeanos explorer 2011 field season," Oceanography, vol. 25, no. 1, 2012.

[127] A. Madanayake, R. Wimalagunaratne, D. G. Dansereau, and L. T. Bruton, "Design and FPGA-implementation of 1st-order 4D IIR frequency-hyperplanar digital filters," in IEEE Int. Midwest Symp. Circuits and Systems (MWSCAS), Aug. 2011

[128] A. Madanayake, R. Wimalagunaratne, D. G. Dansereau, and L. T. Bruton, "A systolic-array architecture for first-order 4-D IIR frequencyplanar digital filters," in IEEE Int. Symp. Circuits and Systems (ISCAS), May 2012, pp. 3069-3072. 